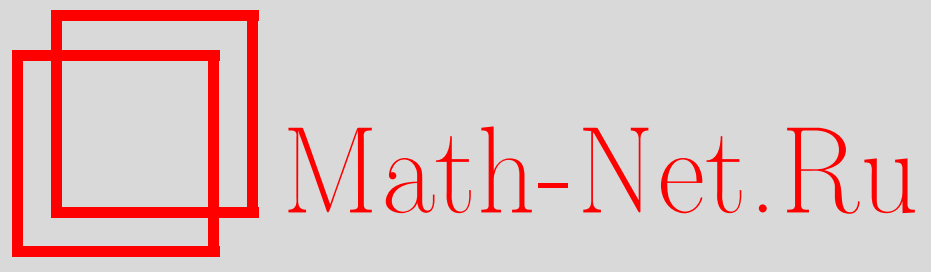

С. В. Зелик, А. А. Ильин, Асимптотика функций Грина и точные интерполяционные неравенства, УМН, 2014, том 69, выпуск 2, 23-76

DOI: https://doi.org/10.4213/rm9575

Использование Общероссийского математического портала Math-Net.Ru подразумевает, что вы прочитали и согласны с пользовательским соглашением http://www . mathnet.ru/rus/agreement

Параметры загрузки:

IP : 34.227 .88 .159

26 апреля 2023 г., 14:02:07

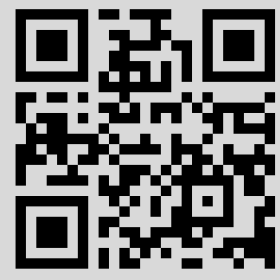




\title{
Асимптотика функций Грина и точные интерполяционные неравенства
}

\author{
С. В. Зелик, А. А. Ильин
}

Предложен общий метод нахождения точных постоянных во вложениях пространств Соболева $H^{m}(\mathscr{M})$, определенных на $n$-мерном римановом многообразии $\mathscr{M}$, в пространство ограниченных непрерывных функций при $m>n / 2$. Метод основан на анализе асимптотического поведения по отношению к спектральному параметру функции Грина эллиптического оператора порядка $2 m$, область определения квадратного корня из которого определяет норму соответствующего пространства Соболева. Подробно рассматриваются $n$-мерный тор $\mathbb{T}^{n}$ и $n$-мерная сфера $\mathbb{S}^{n}$, а также некоторые многообразия с границей. В определенных случаях, когда $\mathscr{M}$ компактно, получены мультипликативные неравенства с остаточными членами различного вида (корректорами). Из неравенств с корректорами для периодических функций выводятся неравенства, улучшающие известные неравенства Карлсона.

Библиография: 28 названий.

Ключевые слова: неравенства Соболева, интерполяционные неравенства, функции Грина, точные постоянные, неравенство Карлсона.

DOI: $10.4213 / \mathrm{rm} 9575$

\section{СОДЕРЖАНИЕ}

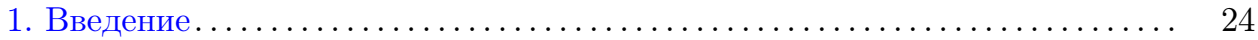

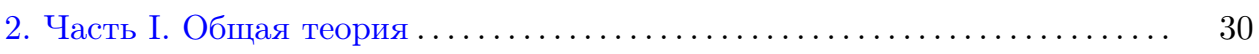

2.1. Предположения и предварительные сведения ............... 30

2.2. Интерполяционное неравенство и вариационная задача ....... 31

2.3. Функции Грина и воспроизводящие функционалы .......... 32

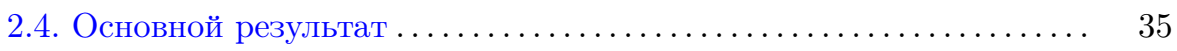

2.5. Асимптотическое разложение при больших $\lambda \ldots \ldots \ldots \ldots \ldots \ldots . . \ldots 39$

2.6. Вариационное представление $\mathbb{V}(\xi, \lambda) \ldots \ldots \ldots \ldots \ldots \ldots \ldots \ldots \ldots . \ldots \ldots$

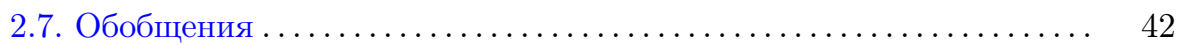

Работа выполнена при поддержке РФФИ (гранты № 12-01-00203, 11-01-00339), Министерства образования и науки РФ (соглашение № 8502) и Программы РАН № 1.

(C) С. В. ЗЕлик, А. А. Ильин, 2014 
3. Часть II. Примеры и приложения ......................... 43

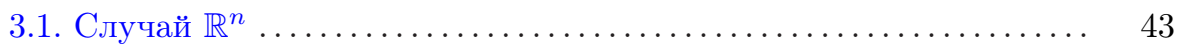

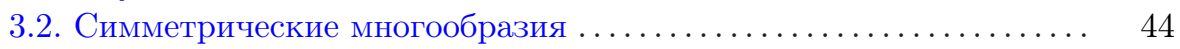

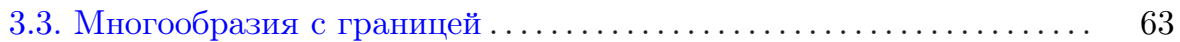

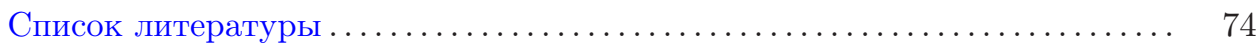

\section{1. Введение}

В этой работе предложен общий метод нахождения точных постоянных в мультипликативных неравенствах Соболева, Гальярдо, Ниренберга, описывающих вложение гильбертовых пространств Соболева $H^{m}(\mathscr{M})$ в пространство ограниченных непрерывных функций $C(\mathscr{M})$. Здесь $\mathscr{M}-n$-мерное многообразие и $m>n / 2$. Рассматриваются следующие интегральные неравенства:

$$
\|u\|_{L_{\infty}(\mathscr{M})}^{2} \leqslant K\|u\|_{H^{l}(\mathscr{M})}^{2 \theta}\|u\|_{H^{m}(\mathscr{M})}^{2(1-\theta)},
$$

где $-\infty<l<n / 2<m<\infty$, так что $0<\theta=\frac{2 m-n}{2(m-l)}<1$.

Известно, конечно, что мультипликативные неравенства верны в гораздо более общей $L_{p}$-постановке (см., например, [3], [26]), где три нормы в (1.1) суть $L_{p^{-}}, L_{q^{-}}$и $L_{r}$-нормы, $1 \leqslant p, q, r \leqslant \infty$, а $\theta$ соответствующим образом определена по масштабной инвариантности; в этом смысле в данной работе мы рассматриваем только случай $L_{\infty}-L_{2}-L_{2}$. В одномерном случае, когда $\mathscr{M}=\mathbb{R}$, $\mathbb{R}_{+}$или $(0, L)$, соответствующие интерполяционные неравенства называются неравенствами для производных. Им посвящена обширная литература (см., например, [1], [21] и библиографию в этих работах). На всей прямой общий $L_{p}-L_{q}-L_{r}$-случай функции и первой производной исчерпывающим образом рассмотрен в [23].

Точная постоянная в неравенстве (1.1) на прямой $\mathbb{R}$ была найдена в [24], более сложный случай полупрямой $\mathbb{R}_{+}$разобран в [11], а явное выражение для точной постоянной в этом случае получено в [17].

Точная постоянная в неравенстве (1.1) для периодических функций с нулевым средним была найдена в [14]. В частности, для $l=0$ там было показано, чTO

$$
\|u\|_{\infty}^{2} \leqslant K(m)\|u\|^{2 \theta}\left\|u^{(m)}\right\|^{2(1-\theta)}, \quad u \in H_{\mathrm{per}}^{m}(0,2 \pi), \quad \int_{0}^{2 \pi} u(x) d x=0,
$$

где $m>1 / 2, \theta=1-1 /(2 m)$ и

$$
K(m)=\left(2 m \theta^{\theta}(1-\theta)^{1-\theta} \sin \pi \theta\right)^{-1} .
$$

Постоянная (которая здесь такая же, как и в случае всей оси) точная, а экстремальной функции нет. Заметим также, что неравенство первого порядка, а именно

$$
\|u\|_{\infty}^{2} \leqslant\|u\|\left\|u^{\prime}\right\|, \quad u \in H_{\mathrm{per}}^{1}(0,2 \pi), \quad \int_{0}^{2 \pi} u(x) d x=0
$$


было получено на самом деле значительно раньше в [13] в качестве доказательства неравенства Карлсона. (В конце этого раздела мы более подробно обсудим связь наших результатов с неравенством Карлсона.)

Точные постоянные в неравенствах (1.1) на сфере $\mathbb{S}^{n}$ были найдены в [15].

Детальное исследование точных констант в неравенстве (1.1) и в логарифмическом неравенстве Брезиса-Галлуэ на $n$-мерном торе $\mathbb{T}^{n}$ было проведено в [2], где впервые были получены неравенства с корректорами. Например, верны следующие неравенства:

$$
\begin{array}{lll}
\|u\|_{\infty}^{2} \leqslant\|u\|\left\|u^{\prime}\right\|-\frac{1}{\pi}\|u\|^{2}, & u \in H_{\mathrm{per}}^{1}(0,2 \pi), & \int_{0}^{2 \pi} u(x) d x=0, \\
\|u\|_{\infty}^{2} \leqslant \frac{\sqrt{2}}{\sqrt[4]{27}}\|u\|^{3 / 2}\left\|u^{\prime \prime}\right\|^{1 / 2}-\frac{2}{3 \pi}\|u\|^{2}, & u \in H_{\mathrm{per}}^{2}(0,2 \pi), & \int_{0}^{2 \pi} u(x) d x=0,
\end{array}
$$

где все постоянные точные, а экстремальных функций нет. Эти неравенства были получены в [2] с существенным использованием компьютерных вычислений. Чисто аналитическое доказательство их получено в данной работе в качестве одного из приложений (см. теорему 3.3).

В первой части работы описан общий метод нахождения точных постоянных в неравенствах вида (1.1), включая и неравенства с корректорами. А именно, пусть $A$ и $B$ суть самосопряженные неотрицательные эллиптические дифференциальные операторы на $\mathscr{M}$ порядка $2 m$ и $2 l$ соответственно. (Для описания основной идеи можно во введении временно предположить, что $l=0$ и $B=\mathrm{Id}$.) В силу эллиптической регулярности ядро $A$ конечномерно и состоит из гладких (ортонормированных) функций:

$$
\operatorname{ker} A=\operatorname{span}\left\{\varphi_{1}, \ldots, \varphi_{k}\right\} .
$$

Обозначим $\bar{H}^{m}:=H^{m}(\mathscr{M}) \cap \operatorname{ker} A^{\perp}$ и $\bar{H}^{l}:=H^{l}(\mathscr{M}) \cap \operatorname{ker} A^{\perp}$. Тогда квадратные корни из $A$ и $B$ определяют эквивалентные нормы на $\bar{H}^{m}(\mathscr{M})$ и $\bar{H}^{l}(\mathscr{M})$ :

$$
\|u\|_{A}^{2}:=(A u, u) \sim\|u\|_{H^{m}(\mathscr{M})}^{2}, \quad\|u\|_{B}^{2}:=(B u, u) \sim\|u\|_{H^{l}(\mathscr{M})}^{2}
$$

для $u \in \bar{H}^{m}$ и $u \in \bar{H}^{l}$ соответственно.

Зафиксируем произвольную точку $\xi \in \mathscr{M}$ и рассмотрим следующий аналог неравенства (1.1):

$$
|u(\xi)|^{2} \leqslant K\|u\|_{B}^{2 \theta}\|u\|_{A}^{2(1-\theta)},
$$

в котором нас интересует точная постоянная $K=K(\xi)$. Для этого мы вводим следующую экстремальную задачу: для любого $D \geqslant \lambda_{0}$, где $\lambda_{0}$ - первое собственное значение оператора $B^{-1 / 2} A B^{-1 / 2}$ в $\bar{H}:=L_{2}(\mathscr{M}) \cap \operatorname{ker} A^{\perp}$, найти $\mathbb{V}(\xi, D)$ - решение следующей задачи максимизации:

$$
\mathbb{V}(\xi, D):=\sup \left\{|u(\xi)|^{2}: u \in \bar{H}^{m},\|u\|_{B}^{2}=1,\|u\|_{A}^{2}=D\right\} .
$$

Тогда выполнено неравенство

$$
|u(\xi)|^{2} \leqslant\|u\|_{B}^{2} \mathbb{V}\left(\xi, \frac{\|u\|_{A}^{2}}{\|u\|_{B}^{2}}\right)
$$


и по определению $\mathbb{V}$ есть наименьшая функция, для которой оно выполнено. В частности, если решена задача (1.5), то нахождение наилучшей постоянной $K$ в (1.4) сводится к нахождению наименьшего $K$, для которого выполнено следующее неравенство:

$$
\mathbb{V}(\xi, D) \leqslant K D^{1-\theta}, \quad D \geqslant \lambda_{0}
$$

Решение задачи максимизации (1.5) выражается в терминах функции Грина следующего эллиптического оператора порядка $2 m$ :

$$
\mathbb{A}(\lambda):=A+\lambda B, \quad \lambda>-\lambda_{0}
$$

А именно, пусть $G_{\lambda}(x, \xi)$ - его функция Грина в $\bar{H}$ :

$$
\mathbb{A}(\lambda) G_{\lambda}(\cdot, \xi)=\bar{\delta}(\cdot, \xi), \quad \bar{\delta}(x, \xi):=\delta(x, \xi)-\sum_{j=1}^{k} \varphi_{j}(x) \varphi_{j}(\xi)
$$

где $\delta(x, \xi)$ - дельта-функция Дирака. Из эллиптической регулярности следует, что $G_{\lambda}(\cdot, \xi) \in \bar{H}^{m} \subset C(\mathscr{M})$. Тогда, как показано в теореме 2.1 , в задаче $(1.5)$ существует единственная экстремальная функция

$$
u_{\lambda(D), \xi}(x)=\frac{G_{\lambda(D)}(x, \xi)}{\left\|G_{\lambda(D)}(\cdot, \xi)\right\|_{B}}, \quad \text { так что } \quad \mathbb{V}(\xi, D)=\frac{G_{\lambda(D)}(\xi, \xi)^{2}}{\left\|G_{\lambda(D)}(\cdot, \xi)\right\|_{B}^{2}}
$$

где $\lambda(D)$ есть монотонно возрастающая функция, удовлетворяющая соотношениям $\lim _{D \rightarrow \infty} \lambda(D)=\infty, \lim _{D \rightarrow \lambda_{0}} \lambda(D)=-\lambda_{0}$ и определенная как единственное решение уравнения

$$
\left\|u_{\lambda(D), \xi}\right\|_{A}^{2}=D
$$

Кроме того, как показано в теореме 2.2 , точная постоянная $K$ в мультипликативном неравенстве (1.4) выражается в терминах функции Грина следующим образом:

$$
K=K(\xi):=\frac{1}{\theta^{\theta}(1-\theta)^{1-\theta}} \cdot \sup _{\lambda>0}\left\{\lambda^{\theta} G_{\lambda}(\xi, \xi)\right\}<\infty .
$$

Экстремальная функция $u_{*}$ существует в том и только том случае, когда супремум по $\lambda$ достигается в конечной точке $\lambda_{*}<\infty$, и тогда $u_{*}(x)=\operatorname{const} G_{\lambda_{*}}(x, \xi)$. Заметим, что подход, использовавшийся в [14], [15] в случае сферы $\mathbb{S}^{n}, n \geqslant 1$, в конечном счете сводился к такой же одномерной задаче максимизации.

Последующие результаты (неравенства с корректорами) основаны на знании асимптотического поведения функции Грина при $\lambda \rightarrow \infty$ :

$$
G_{\lambda}(\xi, \xi)=\lambda^{-1}\left(g_{1} \lambda^{1-\theta}+g_{2}+g_{3} \lambda^{\theta-1}+o\left(\lambda^{\theta-1}\right)\right),
$$

где $g_{1}=g_{1}(\xi)>0, g_{2}=g_{2}(\xi), g_{3}=g_{3}(\xi)$ - некоторые известные величины. Это разложение постулируется, и его проверка для конкретных примеров является основной технической задачей. 
При выполнении (1.6) в предложении 2.1 доказывается, что решение задачи (1.5) имеет следующую асимптотику при $D \rightarrow \infty$ :

$$
\begin{gathered}
\mathbb{V}(\xi, D)=g_{1} S D^{1-\theta}+g_{2} \frac{1}{\theta}-\frac{1}{2} S^{-1} \frac{g_{2}^{2}(1-\theta)-2 \theta g_{1} g_{3}}{\theta^{3} g_{1}} D^{\theta-1}+o\left(D^{\theta-1}\right), \\
S:=\frac{1}{\theta^{\theta}(1-\theta)^{1-\theta}} .
\end{gathered}
$$

Предположим, что третий член в (1.7) отрицательный (это всегда так при $\left.g_{3} \leqslant 0\right)$. Тогда

$$
\mathbb{V}(\xi, D)<g_{1} S D^{1-\theta}+\frac{g_{2}}{\theta}
$$

при больших $D$. В некоторых примерах во второй части статьи мы увидим, что это неравенство выполнено при всех $D$. Это означает, что верно мультипликативное неравенство с корректором (который отрицателен, если $g_{2}<0$ ):

$$
|u(\xi)|^{2} \leqslant g_{1} S\|u\|_{B}^{\theta}\|u\|_{A}^{1-\theta}+\frac{g_{2}}{\theta}\|u\|_{B}^{2},
$$

где все константы точные.

В конце первой части работы в теореме 2.3 приводится следующее вариационное представление $\mathbb{V}(\xi, D)$ :

$$
\mathbb{V}(\xi, D)=\inf _{\lambda \in\left[-\lambda_{0}, \infty\right)}\left\{(\lambda+D) G_{\lambda}(\xi, \xi)\right\}
$$

Эта формула очень полезна для доказательства в отдельных (немногочисленных) случаях, что неравенство (1.8) выполнено для всех $D$. Схема такова. Обычно невозможно найти точку минимума $\lambda(D)$ в явном виде, но в любом случае для нее есть асимптотическая формула

$$
\lambda(D)=\frac{\theta}{1-\theta} D+\frac{g_{2}}{g_{1}} S D^{\theta}+\cdots
$$

(см. (2.44)). Каким-то образом усечем это разложение и обозначим результат через $\lambda_{*}(D)$. Тогда для доказательства (1.8) мы поступаем следующим образом:

$$
\mathbb{V}(\xi, D)-g_{1} S D^{1-\theta}-\frac{g_{2}}{\theta} \leqslant\left(\lambda_{*}(D)+D\right) G_{\lambda_{*}(D)}(\xi, \xi)-g_{1} S D^{1-\theta}-\frac{g_{2}}{\theta} .
$$

Теперь для фиксированного $\xi$ правая часть является функцией только переменной $D$, и более или менее стандартные методы могут быть использованы для доказательства отрицательности правой части для всех $D$. На самом деле неравенства (1.3), а также многие неравенства с корректорами, которые приводятся ниже, доказываются следуя этой схеме.

Во второй части статьи рассматриваются примеры и приложения общего подхода, описанного в первой части, и описанная выше схема используется в теореме 3.3 для чисто аналитического доказательства неравенств (1.3) (доказательство в [2] содержало некоторые достоверные вычисления на компьютере). 
Сначала мы имеем дело с многообразиями без края и рассматриваем случай $A=(-\Delta)^{m}, B=(-\Delta)^{l}$, где $\Delta$ - оператор Лапласа-Бельтрами, и $\bar{H}^{s}(\mathscr{M})=$ $H^{s}(\mathscr{M}) \cap\left\{\int_{\mathscr{M}} u(x) d \mathscr{M}=0\right\}$. Асимптотическая формула (1.6) для функции Грина на торе $\mathbb{T}^{n}$ получена с помощью формулы суммирования Пуассона. Это работает для $l=0$. Для $l>0$ имеется особенность, которая устраняется дифференцированием. Это в свою очередь порождает проблему нахождения постоянных интегрирования. Соответствующая техника была предложена в [2] и далее развивается в данной работе. Мы рассматриваем только один пример $n=3, m=2, l=1$ и доказываем следующее неравенство на трехмерном торе $\mathbb{T}^{3}$ :

$$
\|u\|_{\infty}^{2} \leqslant \frac{1}{2 \pi}\|\nabla u\|\|\Delta u\|-\frac{-\beta_{3}}{4 \pi^{3}}\|\nabla u\|^{2}, \quad u \in \bar{H}^{2}\left(\mathbb{T}^{3}\right)
$$

где постоянная интегрирования $\beta_{3}$ выражается в терминах сверхэкспоненциально сходящегося ряда, $\beta_{3}=-8.91363291758515127$. Обе постоянные точные, а экстремальных функций нет.

Далее мы рассматриваем неравенства на сферах. На $\mathbb{S}^{2}$ рассматривается случай, когда $A=(-\Delta)^{m}, m>1 / 2$ и $B=I$. Соответствующая функция Грина на диагонали не зависит от $\xi$ и представляется рядом

$$
G(\lambda)=\frac{1}{4 \pi} \mu^{m} \sum_{n=1}^{\infty}(2 n+1) \varphi(\mu n(n+1)), \quad \text { где } \mu=\lambda^{-1 / m}, \varphi(x)=\frac{1}{x^{m}+1} .
$$

Таким образом, нам надо изучить асимптотическое поведение функций вида

$$
F(\mu)=\sum_{n=1}^{\infty}(2 n+1) f(\mu n(n+1)) \quad \text { при } \mu \rightarrow 0,
$$

где $f$ достаточно гладкая и достаточно быстро убывает на бесконечности. С использованием формулы суммирования Эйлера-Маклорена это сделано в леммe 3.5:

$$
F(\mu)=\frac{1}{\mu} \int_{0}^{\infty} f(x) d x-\frac{2}{3} f(0)-\frac{1}{15} \mu f^{\prime}(0)+O\left(\mu^{2}\right)
$$

Этим получено асимптотическое разложение вида (1.6) функции Грина и, стало быть, асимптотическое разложение (1.7) решения $\mathbb{V}(D)$ соответствующей экстремальной задачи, в котором второй и третий члены оказываются оба отрицательными. Поэтому может существовать отрицательный корректор. Для $m=2$ мы показываем, что это действительно так и справедливо следующее неравенство (в котором обе постоянные точные и экстремальной функции нет): для $u \in \bar{H}^{2}\left(\mathbb{S}^{2}\right)$

$$
\|u\|_{\infty}^{2} \leqslant \frac{1}{4}\|u\|\|\Delta u\|-\frac{1}{3 \pi}\|u\|^{2} .
$$

Для бо́льших $m(m=3, m=4)$ отрицательный корректор также существует, но он меньше, чем второй член в разложении $\mathbb{V}(D)$. 
На $\mathbb{S}^{3}$ рассматривается только один пример с $A=(-\Delta)^{2}$ и $B=-\Delta$. Ряд, выражающий функцию Грина, суммируется в явном виде, и, действуя аналогичным образом, получаем следующее точное неравенство для $u \in \bar{H}^{2}\left(\mathbb{S}^{3}\right)$ :

$$
\|u\|_{\infty}^{2} \leqslant \frac{1}{2 \pi}\|\nabla u\|\|\Delta u\|-\frac{3}{4 \pi^{2}}\|\nabla u\|^{2} .
$$

В оставшейся части статьи мы рассматриваем многообразия с краем. Сначала доказывается точное мультипликативное неравенство для оператора Бесселя на полупрямой [10] и на конечном интервале.

Затем рассматривается неравенство для $u \in H_{0}^{1}(0, L)$. Корректор существует, но является экспоненциально малым, а именно, верно следующее неравенство:

$$
\|u\|_{\infty}^{2} \leqslant\|u\|\left\|u^{\prime}\right\|\left(1-2 e^{-L\left\|u^{\prime}\right\| /\|u\|}\right) .
$$

Коэффициенты двух слагаемых справа точные, и экстремальной функции нет.

Для неравенства второго порядка

$$
\|u\|_{\infty}^{2} \leqslant K\|u\|_{L_{2}(D)}^{3 / 2}\left\|u^{\prime \prime}\right\|_{L_{2}(D)}^{1 / 2}
$$

на полуоси $D=\mathbb{R}_{+}$и на интервале $D=(0, L)$, если $u \in H_{0}^{2}(D)$, то можно использовать продолжение нулем, что дает константу $K$ такую же, как и на всей оси $\mathbb{R}$, а именно $K=\sqrt{2} / \sqrt[4]{27}$ (см. [24], а также (3.2)). При переходе к более широкому пространству $u \in H_{0}^{1}(D) \cap H^{2}(D)$ константа может возрасти. В теореме 3.9 показано, что это действительно происходит:

$$
\begin{aligned}
& K_{\mathbb{R}_{+}}=\frac{\sqrt{2}}{\sqrt[4]{27}} \cdot\left(1+e^{-\pi}\right)=\frac{\sqrt{2}}{\sqrt[4]{27}} \cdot 1.0432 \ldots, \\
& K_{\text {int }}=\frac{\sqrt{2}}{\sqrt[4]{27}} \cdot \operatorname{coth} \frac{\pi}{2}=\frac{\sqrt{2}}{\sqrt[4]{27}} \cdot 1.09033 \ldots
\end{aligned}
$$

В некотором смысле противоположный результат получен в теореме 3.10 : константа $1 /(2 \pi)$ в неравенстве

$$
\|u\|_{\infty}^{2} \leqslant \frac{1}{2 \pi}\|\nabla u\|\|\Delta u\|
$$

точная как для $u \in H_{0}^{2}(\Omega)$, так и для $u \in H_{0}^{1}(\Omega) \cap H^{2}(\Omega), \Omega \subset \mathbb{R}^{3}$.

В заключение отметим, что неравенства вида (1.1) находят применение в различных задачах теории уравнений с частными производными и математической физики. Например, далеко идущее обобщение на матричный случай [6] простейшего неравенства

$$
\|u\|^{2} \leqslant\|u\|\left\|u^{\prime}\right\|, \quad u \in H^{1}(\mathbb{R})
$$

дает наилучшие на сегодняшний день оценки постоянных в неравенствах Либа-Тирринга для отрицательного следа операторов Шрёдингера в $\mathbb{R}^{n}[20]$.

Соответственно, неравенства с корректорами (1.3) дают по методу [7] одновременную оценку для отрицательного следа и числа отрицательных собственных значений оператора Шрёдингера на $\mathbb{S}^{1}[16]$. 
Наконец, неравенства (1.3) улучшают известное неравенство Карлсона [5] (см. также [19] и цитированную там литературу): для $a_{k} \geqslant 0$

$$
\left(\sum_{k=1}^{\infty} a_{k}\right)^{2} \leqslant \pi\left(\sum_{k=1}^{\infty} a_{k}^{2}\right)^{1 / 2}\left(\sum_{k=1}^{\infty} k^{2} a_{k}^{2}\right)^{1 / 2}
$$

где постоянная $\pi$ точная, и неравенство строгое, если не все $a_{k}$ равны 0 . Действительно, как показано в [13], неравенства (1.2) и (1.9) эквивалентны, поэтому улучшение (1.2) дает улучшение (1.9).

Для заданной последовательности $a_{k} \geqslant 0$ положим $a_{0}=0$ и рассмотрим вещественную функцию

$$
u(x)=\sum_{k=-\infty}^{\infty} a_{|k|} e^{i k x}
$$

с нулевым средним, для которой

$$
\|u\|_{\infty}=u(0)=2 \sum_{k=1}^{\infty} a_{k}, \quad\|u\|^{2}=4 \pi \sum_{k=1}^{\infty} a_{k}^{2}, \quad\left\|u^{\prime}\right\|^{2}=4 \pi \sum_{k=1}^{\infty} k^{2} a_{k}^{2} .
$$

Тогда неравенства (1.3) можно переформулировать в виде

$$
\begin{aligned}
& \left(\sum_{k=1}^{\infty} a_{k}\right)^{2} \leqslant \pi\left(\sum_{k=1}^{\infty} a_{k}^{2}\right)^{1 / 2}\left(\sum_{k=1}^{\infty} k^{2} a_{k}^{2}\right)^{1 / 2}-\sum_{k=1}^{\infty} a_{k}^{2}, \\
& \left(\sum_{k=1}^{\infty} a_{k}\right)^{2} \leqslant \frac{\sqrt{2} \pi}{\sqrt[4]{27}}\left(\sum_{k=1}^{\infty} a_{k}^{2}\right)^{3 / 4}\left(\sum_{k=1}^{\infty} k^{4} a_{k}^{2}\right)^{1 / 4}-\frac{2}{3} \sum_{k=1}^{\infty} a_{k}^{2},
\end{aligned}
$$

где все постоянные точные, а неравенства строгие, если не все $a_{k}$ равны 0. Более того, интерполяционные неравенства на торах $\mathbb{T}^{n}$, о которых шла речь выше, могут естественным образом считаться многомерными обобщениями неравенства Карлсона.

В статье используются общепринятые обозначения. Иногда $C$-норма (или $L_{\infty}$-норма) обозначается через $\|\cdot\|_{\infty}$, а $L_{2}$-норма - через $\|\cdot\|$.

\section{2. Часть I. Общая теория}

2.1. Предположения и предварительные сведения. Пусть $\mathscr{M}$ - компактное $n$-мерное риманово многообразие, и пусть

$$
(u, v):=\int_{\mathscr{M}} u(x) v(x) \operatorname{mes}(d x)
$$

обозначает стандартное скалярное произведение в $H:=L^{2}(\mathscr{M}, \mathrm{mes})$ (где mes есть мера на $\mathscr{M}$, порожденная римановой метрикой). Как обычно, определим пространства Соболева $W_{p}^{l}(\mathscr{M}), 1 \leqslant p \leqslant \infty$, как пространства распределений, обобщенные производные которых до порядка $l$ принадлежат $L^{p}(\mathscr{M})$ (это определение годится для целых $l$; для нецелых и/или отрицательных $l$ пространства $W_{p}^{l}(\mathscr{M})$ определяются обычным образом с помощью интерполяции 
и двойственности, см., например, [26]). В гильбертовом случае $p=2$ обозначим через $H^{m}=H^{m}(\mathscr{M})$ пространство Соболева $W_{2}^{m}(\mathscr{M})$.

Пусть $A$ есть эллиптический самосопряженный дифференциальный оператор на $\mathscr{M}$ порядка $2 m$ с гладкими коэффициентами, который к тому же неотрицательно определен:

$$
(A u, u) \geqslant 0, \quad u \in H^{m},
$$

хотя может иметь и непустое ядро. В силу классической теории эллиптической регулярности (см., например, [26]), ядро конечномерно и порождено гладкими функциями,

$$
\operatorname{ker} A:=\operatorname{span}\left\{\varphi_{1}, \ldots, \varphi_{k}\right\}, \quad \varphi_{i} \in C^{\infty}(\mathscr{M}), \quad A \varphi_{i}=0,
$$

которые без потери общности считаем ортонормированными: $\left(\varphi_{i}, \varphi_{j}\right)=\delta_{i j}$.

Обозначим через $\bar{H}$ ортогональное дополнение к $\operatorname{ker} A$ в $H=L^{2}(\mathscr{M})$ и положим

$$
\bar{H}^{s}:=H^{s}(\mathscr{M}) \cap \bar{H}, \quad s \in \mathbb{R} .
$$

В силу теории эллиптической регулярности $A$ осуществляет изоморфизм между $\bar{H}^{s+2 m}$ и $\bar{H}^{s}$ для всех $s \in \mathbb{R}$, и, в частности, эквивалентная норма в $\bar{H}^{m}$ есть

$$
\|u\|_{A}^{2}:=(A u, u), \quad u \in \bar{H}^{m} .
$$

Также на $\mathscr{M}$ вводится второй неотрицательный самосопряженный эллиптический оператор $B$ с гладкими коэффициентами порядка $2 l<2 m$ такой, что

$$
\operatorname{ker} B \subset \operatorname{ker} A \quad \text { и } \quad B \operatorname{ker} A \subset \operatorname{ker} A .
$$

Тогда $B$ осуществляет изоморфизм между $\bar{H}^{s+2 l}$ и $\bar{H}^{s}$ для всех $s \in \mathbb{R}$, и, в частности, эквивалентная норма в $\bar{H}^{l}$ есть

$$
\|u\|_{B}^{2}:=(B u, u), \quad u \in \bar{H}^{l} .
$$

Кроме того, справедлив аналог неравенства Пуанкаре:

$$
\|u\|_{B}^{2} \leqslant \lambda_{0}^{-1}\|u\|_{A}^{2}, \quad u \in \bar{H}^{m},
$$

где $\lambda_{0}>0$ - первое собственное значение $B^{-1 / 2} A B^{-1 / 2}$ в $\bar{H}$.

2.2. Интерполяционное неравенство и вариационная задача. Если

$$
l<\frac{n}{2}<m
$$

то для любого $u \in \bar{H}^{m}$ верно следующее интерполяционное неравенство:

$$
\|u\|_{C(\mathscr{M})} \leqslant C\|u\|_{B}^{\theta}\|u\|_{A}^{1-\theta}, \quad \frac{n}{2}=l \theta+m(1-\theta) .
$$

Это неравенство в рассматриваемом гильбертовом случае несложно доказать с помощью локализации и преобразования Фурье. Можно также воспользоваться вложением пространства Бесова $B_{2,1}^{n / 2}$ в пространство непрерывных 
функций, вещественной интерполяцией $B_{2,1}^{n / 2}=\left(B_{2,2}^{m}, B_{2,2}^{l}\right)_{\theta, 1}=\left(H^{m}, H^{l}\right)_{\theta, 1}$ $[3],[26]$ и установленной эквивалентностью норм:

$$
\|u\|_{C} \leqslant c_{1}\|u\|_{B_{2,1}^{n / 2}} \leqslant c_{2}\|u\|_{B_{2,2}^{l}}^{\theta}\|u\|_{B_{2,2}^{m}}^{1-\theta}=c_{2}\|u\|_{H^{l}}^{\theta}\|u\|_{H^{m}}^{1-\theta} \leqslant c_{3}\|u\|_{B}^{\theta}\|u\|_{A}^{1-\theta} .
$$

Впрочем, в рассматриваемых во второй части статьи примерах неравенство (2.9) легко доказывается непосредственно.

Наша цель - уточнить неравенство (2.9) как в смысле нахождения точной постоянной $C=C(n, m, l, \mathscr{M})$, так и в смысле нахождения в нем возможных корректирующих членов. Для этого зафиксируем произвольную точку $\xi \in \mathscr{M}$, положительное число $D$ и рассмотрим следующую задачу максимизации:

$$
\mathbb{V}(\xi, D):=\sup \left\{|u(\xi)|^{2}: u \in \bar{H}^{m},\|u\|_{B}^{2}=1,\|u\|_{A}^{2}=D\right\} .
$$

Действительно, ввиду (2.9) и (2.7) функция $\mathbb{V}$ корректно определена для $\xi \in \mathscr{M}$ и любого $D \geqslant \lambda_{0}$. С другой стороны, из соображений однородности

$$
|u(\xi)|^{2} \leqslant\|u\|_{B}^{2} \mathbb{V}\left(\xi, \frac{\|u\|_{A}^{2}}{\|u\|_{B}^{2}}\right), \quad \xi \in \mathscr{M}, \quad u \in \bar{H}^{m} .
$$

В частности, наилучшая постоянная $C$ в $(2.9)$ есть наименьшая постоянная $C$, для которой неравенство

$$
\sup _{\xi \in \mathscr{M}} \mathbb{V}(\xi, D) \leqslant C^{2} D^{1-\theta}
$$

выполняется для всех $D \geqslant \lambda_{0}$. Таким образом, изучение неравенства $(2.9)$ сводится к исследованию экстремальной задачи (2.10).

2.3. Функции Грина и воспроизводящие функционалы. В этом разделе описываются некоторые технические средства для аналитического описания функции $\mathbb{V}$ в терминах функции Грина одного семейства эллиптических операторов на $\mathscr{M}$. А именно, положим

$$
\mathbb{A}(\lambda):=A+\lambda B, \quad \lambda>-\lambda_{0},
$$

и пусть функция $G_{\lambda}(x, \xi)$ есть решение уравнения

$$
\mathbb{A}(\lambda) G_{\lambda}(\cdot, \xi)=\bar{\delta}(\cdot, \xi), \quad \bar{\delta}(x, \xi)=\delta(x, \xi)-\sum_{i=1}^{k} \varphi_{i}(\xi) \varphi_{i}(x),
$$

где $\delta(x, \xi)$ - это $\delta$-функция в точке $\xi \in \mathscr{M}$, а $\bar{\delta}$ есть ее "проекция" на $\bar{H}$. Тогда в силу теоремы вложения Соболева и (2.8) выполняется включение

$$
\bar{\delta}(\cdot, \xi) \in \bar{H}^{-m}
$$

и в силу эллиптической регулярности

$$
G_{\lambda}(\cdot, \xi) \in \bar{H}^{m} \subset C(\mathscr{M}) .
$$


Далее, поскольку $\mathbb{A}(\lambda)$ самосопряжен, то $G_{\lambda}(x, \xi)=G_{\lambda}(\xi, x)$ и, следовательно, из (2.15) вытекает, что

$$
G_{\lambda} \in C(\mathscr{M} \times \mathscr{M})
$$

Кроме того,

$$
u(\xi)=(\bar{\delta}(\cdot, \xi), u)=\left(\mathbb{A}(\lambda) G_{\lambda}(\cdot, \xi), u\right)=\left(G_{\lambda}(\cdot, \xi), \mathbb{A}(\lambda) u\right)
$$

для всех $u \in \bar{H}^{m}$. В частности, выбирая $u(x):=G_{\lambda}(x, \xi)$, получаем

$$
G_{\lambda}(\xi, \xi)=\left(\mathbb{A}(\lambda) G_{\lambda}(\cdot, \xi), G_{\lambda}(\cdot, \xi)\right)=\left\|G_{\lambda}(\cdot, \xi)\right\|_{A}^{2}+\lambda\left\|G_{\lambda}(\cdot, \xi)\right\|_{B}^{2}>0 .
$$

Следующая простая лемма является тем не менее основным техническим средством метода воспроизводящих функционалов и позволит нам найти аналитическое представление для функции $\mathbb{V}$.

ЛЕмма 2.1. Пусть выполнены перечисленные выше предположения. Тогда для любого $u \in \bar{H}^{m}$ и любого $\lambda>-\lambda_{0}$

$$
|u(\xi)|^{2} \leqslant G_{\lambda}(\xi, \xi)\left(\|u\|_{A}^{2}+\lambda\|u\|_{B}^{2}\right) .
$$

Кроме того, равенство здесъ имеет место тогда и толъко тогда, когда и $(x)=$ $c G_{\lambda}(x, \xi)$ для некоторого $c \in \mathbb{R}$.

ДокАзАТЕльство. Действительно, поскольку $\mathbb{A}(\lambda)$ положительно определен, по неравенству Коши-Буняковского-Шварца имеем

$$
\begin{aligned}
|u(\xi)|^{2} & =\left(G_{\lambda}(\cdot, \xi), \mathbb{A}(\lambda) u\right)^{2} \leqslant\left(\mathbb{A}(\lambda) G_{\lambda}, G_{\lambda}\right)(\mathbb{A}(\lambda) u, u) \\
& =G_{\lambda}(\xi, \xi)\left(\|u\|_{A}^{2}+\lambda\|u\|_{B}^{2}\right),
\end{aligned}
$$

причем равенство здесь имеет место, лишь если $u(x)=c G_{\lambda}(x, \xi)$. Лемма доказана.

Обратим внимание, что до сих пор число $\lambda \geqslant-\lambda_{0}$ было свободным параметром в (2.18). В следующей лемме показано, что этот параметр можно выбрать таким образом, что отношение $\left\|G_{\lambda}(\cdot)\right\|_{A}^{2} /\left\|G_{\lambda}(\cdot, \xi)\right\|_{B}^{2}$ будет принимать любое наперед заданное значение.

ЛЕмма 2.2. Функиия

$$
D(\lambda)=D_{\xi}(\lambda):=\frac{\left\|G_{\lambda}(\cdot, \xi)\right\|_{A}^{2}}{\left\|G_{\lambda}(\cdot, \xi)\right\|_{B}^{2}}
$$

строго возрастает на $\left[-\lambda_{0}, \infty\right), u$

$$
\lim _{\lambda \rightarrow-\lambda_{0}} D(\lambda)=\lambda_{0}, \quad \lim _{\lambda \rightarrow+\infty} D(\lambda)=\infty,
$$

так что обратная функиия $D \mapsto \lambda(D)$ корректно определена на $\left[\lambda_{0}, \infty\right)$. 
Доказательство. Покажем, что $D^{\prime}(\lambda)>0$. С этой целью заметим, что функция $G_{\lambda}(x, \xi)$ дифференцируема по $\lambda$ и производная $G_{\lambda}^{\prime}(x, \xi):=\partial_{\lambda} G_{\lambda}(x, \xi)$ удовлетворяет уравнению

$$
A G_{\lambda}^{\prime}(\cdot, \xi)+\lambda B G_{\lambda}^{\prime}(\cdot, \xi)=\mathbb{A}(\lambda) G_{\lambda}^{\prime}(\cdot, \xi)=-B G_{\lambda}(\cdot, \xi)
$$

так что

$$
A G_{\lambda}^{\prime}(\cdot, \xi)=-B G_{\lambda}(\cdot, \xi)-\lambda B G_{\lambda}^{\prime}(\cdot, \xi), \quad G_{\lambda}^{\prime}(\cdot, \xi)=-\mathbb{A}(\lambda)^{-1} B G_{\lambda} .
$$

Поэтому

$$
\begin{aligned}
\frac{1}{2}\left\|G_{\lambda}(\cdot, \xi)\right\|_{B}^{4} D^{\prime}(\lambda)= & \left\|G_{\lambda}(\cdot, \xi)\right\|_{B}^{2}\left(A G_{\lambda}^{\prime}(\cdot, \xi), G_{\lambda}(\cdot, \xi)\right) \\
& -\left(B G_{\lambda}^{\prime}(\cdot, \xi), G_{\lambda}(\cdot, \xi)\right)\left\|G_{\lambda}(\cdot, \xi)\right\|_{A}^{2} \\
= & -\left\|G_{\lambda}(\cdot, \xi)\right\|_{B}^{4}-\lambda\left\|G_{\lambda}(\cdot, \xi)\right\|_{B}^{2}\left(B G_{\lambda}^{\prime}(\cdot, \xi), G_{\lambda}(\cdot, \xi)\right) \\
& -\left(B G_{\lambda}^{\prime}(\cdot, \xi), G_{\lambda}(\cdot, \xi)\right)\left\|G_{\lambda}(\cdot, \xi)\right\|_{A}^{2} \\
= & -\left\|G_{\lambda}(\cdot, \xi)\right\|_{B}^{4}-\left(B G_{\lambda}^{\prime}(\cdot, \xi), G_{\lambda}(\cdot, \xi)\right)\left(\mathbb{A}(\lambda) G_{\lambda}(\cdot, \xi), G_{\lambda}(\cdot, \xi)\right) \\
= & \left(\mathbb{A}(\lambda)^{-1} B G_{\lambda}(\cdot, \xi), B G_{\lambda}(\cdot, \xi)\right)\left(\mathbb{A}(\lambda) G_{\lambda}(\cdot, \xi), G_{\lambda}(\cdot, \xi)\right) \\
& -\left\|G_{\lambda}(\cdot, \xi)\right\|_{B}^{4} .
\end{aligned}
$$

Применяя неравенство Коши-Буняковского-Шварца, имеем

$$
\begin{aligned}
\|v\|_{B}^{4} & =(B v, v)^{2}=\left(\mathbb{A}^{-1 / 2}(\lambda) B v, \mathbb{A}^{1 / 2}(\lambda) v\right)^{2} \\
& \leqslant\left\|\mathbb{A}^{-1 / 2}(\lambda) B v\right\|_{H}^{2}\left\|\mathbb{A}^{1 / 2}(\lambda) v\right\|_{H}^{2}=\left(\mathbb{A}^{-1}(\lambda) B v, B v\right)(\mathbb{A}(\lambda) v, v)
\end{aligned}
$$

для всех $v \in \bar{H}^{m}$. Выбирая $v(x)=G_{\lambda}(x, \xi)$, из $(2.22)$ получаем, что $D^{\prime}(\lambda) \geqslant 0$. Более того, равенство в (2.23) возможно, лишь только если $\mathbb{A}(\lambda) v=\beta B v, \beta \in \mathbb{R}$, что дает

$$
\mathbb{A}(\lambda) v=\mathbb{A}(\lambda) G_{\lambda}(\cdot, \xi)=\bar{\delta}(x, \xi)=\beta B G_{\lambda}(\cdot, \xi) \in \bar{H}^{m-l}, \quad \text { где } m-l>0,
$$

что, конечно, невозможно, и, стало быть, $D^{\prime}(\lambda)>0$. Итак, доказано, что $D(\lambda)$ строго возрастает.

Теперь надо доказать (2.20). Начнем с предела $\lambda \rightarrow-\lambda_{0}$. Для того чтобы решить (2.14) вблизи $\lambda=-\lambda_{0}$, обозначим $w(x, \xi)=B^{1 / 2} G_{\lambda}(x, \xi)$. Тогда

$$
B^{-1 / 2} A B^{-1 / 2} w+\lambda w=B^{-1 / 2} \bar{\delta}(x, \xi),
$$

и, по определению, $\lambda_{0}$ есть наименьшее собственное число самосопряженного положительно определенного оператора $B^{-1 / 2} A B^{-1 / 2}$ в $\bar{H}$. Этот оператор имеет компактную резольвенту, и его спектр дискретный. Пусть $\left\{\psi_{k}\right\}_{k=1}^{\infty}-$ семейство ортонормированных собственных функций, и пусть первые $p(p \geqslant 1)$ из них отвечают минимальному собственному значению $\lambda_{0}$. При этом $\psi_{i} \in C^{\infty}(\mathscr{M})$. Ищем $w$ в виде

$$
w(x, \xi)=\sum_{i=1}^{p} a_{i} \psi_{i}(x)+\sum_{i=p+1}^{\infty} a_{i} \psi_{i}(x)=: w_{0}+w^{\perp} .
$$


Подставляя это в $(2.24)$ и беря скалярное произведение с $\psi_{1}, \ldots, \psi_{p}$, находим коэффициенты $w_{0}$-части решения:

$$
a_{i}=\frac{1}{\lambda+\lambda_{0}}\left(B^{-1 / 2} \bar{\delta}(\cdot, \xi), \psi_{i}\right)=\frac{\widetilde{\psi}_{i}(\xi)}{\lambda+\lambda_{0}}, \quad i=1, \ldots, p
$$

где мы обозначили $\widetilde{\psi}_{i}:=B^{-1 / 2} \psi_{i}$. Заметим, что $w^{\perp}$-часть решения остается ограниченной при $\lambda \rightarrow-\lambda_{0}$. Применяя $B^{-1 / 2}$ к $(2.25)$, находим $G_{\lambda}(x, \xi)$ :

$$
G_{\lambda}(x, \xi)=\frac{1}{\lambda+\lambda_{0}} \sum_{i=1}^{p} \widetilde{\psi}_{i}(\xi) \widetilde{\psi}_{i}(x)+G_{\lambda}^{\perp}(x, \xi)
$$

где часть $G_{\lambda}^{\perp}(x, \xi)$ остается ограниченной при $\lambda \rightarrow-\lambda_{0}$. Итак,

$$
\lim _{\lambda \rightarrow-\lambda_{0}} D(\lambda)=\frac{\left\|\sum_{i=1}^{p} \widetilde{\psi}_{i}(\xi) \widetilde{\psi}_{i}(\cdot)\right\|_{A}^{2}}{\left\|\sum_{i=1}^{p} \widetilde{\psi}_{i}(\xi) \widetilde{\psi}_{i}(\cdot)\right\|_{B}^{2}}=\lambda_{0} \frac{\sum_{i=1}^{p} \widetilde{\psi}_{i}(\xi)^{2}}{\sum_{i=1}^{p} \widetilde{\psi}_{i}(\xi)^{2}}=\lambda_{0} .
$$

Теперь рассмотрим случай, когда $\lambda \rightarrow \infty$. Предположим, что (2.20) неверно и

$$
\lim _{\lambda \rightarrow \infty} D(\lambda)=D_{\max }<\infty
$$

Тогда

$$
\left\|G_{\lambda}(\cdot, \xi)\right\|_{A}^{2} \leqslant D_{\max }\left\|G_{\lambda}(\cdot, \xi)\right\|_{B}^{2} .
$$

Умножая (2.14) на $G_{\lambda}(x, \xi)$, интегрируя по $x \in \mathscr{M}$ и используя вложение $\bar{H}^{m} \subset$ $C$ и неравенство (2.27), получаем

$$
\begin{aligned}
\left\|G_{\lambda}(\cdot, \xi)\right\|_{A}^{2}+\lambda\left\|G_{\lambda}(\cdot, \xi)\right\|_{B}^{2} & =G_{\lambda}(\xi, \xi) \leqslant\left\|G_{\lambda}(\cdot, \xi)\right\|_{C} \\
& \leqslant C\left\|G_{\lambda}(\cdot, \xi)\right\|_{A} \leqslant C_{1}\left\|G_{\lambda}(\cdot, \xi)\right\|_{B}
\end{aligned}
$$

Вместе с (2.27) это дает

$$
\left\|G_{\lambda}(\cdot, \xi)\right\|_{A} \leqslant C\left\|G_{\lambda}(\cdot, \xi)\right\|_{B} \leqslant C_{2} \lambda^{-1},
$$

где $C_{2}$ не зависит от $\lambda \rightarrow \infty$. Таким образом, функция $w_{\lambda}(x, \xi):=\lambda_{\lambda}(x, \xi)$ равномерно ограничена в $\bar{H}^{m}$ и без потери общности можно считать, что $w_{\lambda}(\cdot, \xi) \rightarrow$ $w_{\infty}(\cdot, \xi)$ слабо в этом пространстве. Тогда $w_{\infty}(\cdot, \xi) \in \bar{H}^{m}$ и

$$
B w_{\infty}(\cdot, \xi)=\bar{\delta}(\cdot, \xi) .
$$

Далее, поскольку $w_{\infty} \in \bar{H}^{m}$, то $\bar{\delta}(\cdot, \xi) \in \bar{H}^{m-2 l}$. В силу (2.8) выполняется неравенство $m-2 l>-n / 2$, так что $\delta(\cdot, \xi) \in H^{-s}$ для некоторого $s<n / 2$, что невозможно. Итак, пределы (2.20) существуют, и лемма доказана.

2.4. Основной результат. Цель этого раздела - получение аналитического выражения для функции $\mathbb{V}$ в терминах введенной выше функции Грина. Соответствующий результат приведен в следующей теореме. 
Теорема 2.1. Пусть выполнены перечисленные выше предположения. Тогда для любого $D \in\left[\lambda_{0}, \infty\right)$ и любой точки $\xi \in \mathscr{M}$ супремум в (2.10) есть максимум, достигающийся на единственной функции

$$
u_{\lambda(D), \xi}(x):=\frac{G_{\lambda(D)}(x, \xi)}{\left\|G_{\lambda(D)}(\cdot, \xi)\right\|_{B}},
$$

где функиия $\lambda(D)$ определена в лемме 2.2. В частности,

$$
\mathbb{V}(\xi, D)=\left(\frac{G_{\lambda(D)}(\xi, \xi)}{\left\|G_{\lambda(D)}(\cdot, \xi)\right\|_{B}}\right)^{2}
$$

ДокАЗАТЕЛЬСтво. Пусть $u \in \bar{H}^{m}$ и $\|u\|_{B}=1,\|u\|_{A}^{2}=D$. Тогда по лемме 2.2 существует единственное $\lambda=\lambda(D)$, удовлетворяющее (2.19). Согласно (2.18) с $\lambda=\lambda(D)$ имеем

$$
|u(\xi)|^{2} \leqslant G_{\lambda(D)}(\xi, \xi)(D+\lambda(D)),
$$

причем равенство выполняется, лишь только если $u(x)=c G_{\lambda(D)}(x, \xi)$. Беря $B$-норму от обеих частей этого равенства, находим $c=\left\|G_{\lambda(D)}(\cdot, \xi)\right\|_{B}^{-1}$ и $u(x)=$ $u_{\lambda(D), \xi}(x)$. Теорема доказана.

Таким образом, чтобы найти $\mathbb{V}$, нам нужны три функции

$$
f_{\xi}(\lambda):=G_{\lambda}(\xi, \xi), \quad g_{\xi}(\lambda):=\left\|G_{\lambda}(\cdot, \xi)\right\|_{B}^{2}, \quad h_{\xi}(\lambda):=\left\|G_{\lambda}(\cdot, \xi)\right\|_{A}^{2} .
$$

Тогда

$$
D(\lambda)=\frac{h_{\xi}(\lambda)}{g_{\xi}(\lambda)}, \quad \mathbb{V}(\xi, \lambda):=\mathbb{V}(\xi, D(\lambda))=\frac{f_{\xi}(\lambda)^{2}}{g_{\xi}(\lambda)}, \quad \lambda \in\left[-\lambda_{0}, \infty\right),
$$

и мы имеем параметрическое представление функции $D \mapsto \mathbb{V}(\xi, D)$. В следующей лемме показано, что функции $g$ и $h$ могут быть выражены через $f$.

ЛЕмма 2.3. Функиии $f, g$ и $h$ удовлетворяют следующим равенствам:

$$
f_{\xi}^{\prime}(\lambda):=\frac{d}{d \lambda} f_{\xi}(\lambda)=-g_{\xi}(\lambda), \quad h_{\xi}(\lambda)=f_{\xi}(\lambda)+\lambda f_{\xi}^{\prime}(\lambda) .
$$

ДоказАтельство. Действительно, умножая $(2.14)$ на $G_{\lambda}(x, \xi)$ и интегрируя по $x \in \mathscr{M}$, получаем

$$
f_{\xi}(\lambda)=G_{\lambda}(\xi, \xi)=\left\|G_{\lambda}(\cdot, \xi)\right\|_{A}^{2}+\lambda\left\|G_{\lambda}(\cdot, \xi)\right\|_{B}^{2}=h_{\xi}(\lambda)+\lambda g_{\xi}(\lambda) .
$$

Дифференцируя это равенство по $\lambda$ и используя (2.21), получаем

$$
\begin{aligned}
f_{\xi}^{\prime}(\lambda) & =2\left(\mathbb{A}(\lambda) G_{\lambda}^{\prime}(\cdot, \xi), G_{\lambda}(\cdot, \xi)\right)+g_{\xi}(\lambda) \\
& =-2\left(B G_{\lambda}(\cdot, \xi), G_{\lambda}(\cdot, \xi)\right)+g_{\xi}(\lambda)=-2 g_{\xi}(\lambda)+g_{\xi}(\lambda)=-g_{\xi}(\lambda) .
\end{aligned}
$$

Осталось заметить, что из (2.34) и (2.33) следует (2.32). Лемма доказана.

Следующая теорема говорит о том, что точная постоянная в неравенстве

$$
|u(\xi)|^{2} \leqslant K\|u\|_{B}^{2 \theta}\|u\|_{A}^{2(1-\theta)}, \quad u \in \bar{H}^{m},
$$

выражается в терминах следующей скалярной экстремальной задачи, вновь связанной с функцией Грина $G_{\lambda}(\xi, \xi)$. 
ТЕОрема 2.2. Для фиксированного $\xi \in \mathscr{M}$ постоянная $K$ в (2.35) дается выражением

$$
K=K(\xi):=\frac{1}{\theta^{\theta}(1-\theta)^{1-\theta}} \cdot \sup _{\lambda>0}\left\{\lambda^{\theta} G_{\lambda}(\xi, \xi)\right\}<\infty
$$

где $\theta$ определена в (2.9), и константа (2.36) точная. Кроме того, экстремальная функиия в (2.35) существует тогда и только тогда, когда супремум в (2.36) достигается в конечной точке $\lambda_{*}$.

ДокАзАтЕльство. Убедимся сначала, что правая часть (2.36) конечна. Действительно, в силу (2.17) и неравенства (2.9) (с некоторой не обязательно оптимальной постоянной $C$ ) имеем

$$
\begin{aligned}
\lambda^{\theta} G_{\lambda}(\xi, \xi)^{2} & \leqslant \lambda^{\theta}\left\|G_{\lambda}(\xi, \cdot)\right\|_{\infty}^{2} \leqslant C\left(\lambda\left\|G_{\lambda}(\xi, \cdot)\right\|_{B}^{2}\right)^{\theta}\left(\left\|G_{\lambda}(\xi, \cdot)\right\|_{A}^{2}\right)^{1-\theta} \\
& \leqslant C^{\prime}\left(\left\|G_{\lambda}(\xi, \cdot)\right\|_{A}^{2}+\lambda\left\|G_{\lambda}(\xi, \cdot)\right\|_{B}^{2}\right)=C^{\prime} G_{\lambda}(\xi, \xi)
\end{aligned}
$$

и правая часть (2.36) конечна.

Докажем теперь, что (2.35) выполнено с $K=K(\xi)$. Действительно, пусть $u \in \bar{H}^{m}$ произвольно и

$$
\lambda:=\frac{\theta}{1-\theta} \frac{\|u\|_{A}^{2}}{\|u\|_{B}^{2}} .
$$

Тогда, используя (2.18), получаем

$$
\begin{aligned}
|u(\xi)|^{2} & \leqslant G_{\lambda}(\xi, \xi)\|u\|_{B}^{2}\left(\frac{\|u\|_{A}^{2}}{\|u\|_{B}^{2}}+\lambda\right)=\frac{1}{\theta} G_{\lambda}(\xi, \xi) \lambda\|u\|_{B}^{2} \\
& =\frac{1}{\theta} G_{\lambda}(\xi, \xi) \lambda^{\theta} \lambda^{1-\theta}\|u\|_{B}^{2}=\frac{1}{\theta} \lambda^{\theta} G_{\lambda}(\xi, \xi)\left(\frac{\theta}{1-\theta} \frac{\|u\|_{A}^{2}}{\|u\|_{B}^{2}}\right)^{1-\theta}\|u\|_{B}^{2} \\
& =\frac{1}{\theta^{\theta}(1-\theta)^{1-\theta}} \lambda^{\theta} G_{\lambda}(\xi, \xi)\|u\|_{B}^{2 \theta}\|u\|_{A}^{2(1-\theta)} \\
& \leqslant \frac{1}{\theta^{\theta}(1-\theta)^{1-\theta}} \cdot \sup _{\lambda>0}\left\{\lambda^{\theta} G_{\lambda}(\xi, \xi)\right\}\|u\|_{B}^{2 \theta}\|u\|_{A}^{2(1-\theta)} \\
& =K(\xi)\|u\|_{B}^{2 \theta}\|u\|_{A}^{2(1-\theta)}
\end{aligned}
$$

Докажем точность постоянной $K(\xi)$. Сначала предположим, что супремум в (2.36) есть максимум, достигающийся в точке $\lambda=\lambda_{*}$. Тогда

$$
0=\left.\frac{d}{d \lambda}\left(\lambda^{\theta} f_{\xi}(\lambda)\right)\right|_{\lambda=\lambda_{*}}=\lambda_{*}^{\theta}\left(f_{\xi}^{\prime}\left(\lambda_{*}\right)+\theta \lambda_{*}^{-1} f_{\xi}\left(\lambda_{*}\right)\right)
$$

и $\lambda_{*} f_{\xi}^{\prime}\left(\lambda_{*}\right)+\theta f_{\xi}\left(\lambda_{*}\right)=0$. Поэтому в силу соотношений $(2.32)$

$$
D\left(\lambda_{*}\right)=\frac{h_{\xi}\left(\lambda_{*}\right)}{g_{\xi}\left(\lambda_{*}\right)}=-\frac{f_{\xi}\left(\lambda_{*}\right)+\lambda_{*} f_{\xi}^{\prime}\left(\lambda_{*}\right)}{f_{\xi}^{\prime}\left(\lambda_{*}\right)}=\frac{1-\theta}{\theta} \lambda_{*} .
$$

Итак, $\lambda_{*}=\frac{\theta}{1-\theta} \frac{\left\|G_{\lambda_{*}}(\xi, \cdot)\right\|_{A}^{2}}{\left\|G_{\lambda_{*}}(\xi, \cdot)\right\|_{B}^{2}}$, и все неравенства в $(2.37)$ становятся равенствами, если взять $u(x)=G_{\lambda_{*}}(\xi, x)$, и в этом случае есть точная экстремаль. 
Поскольку $\lambda^{\theta} G_{\lambda}(\xi, \xi) \rightarrow 0$ при $\lambda \rightarrow 0$, то остается лишь рассмотреть случай, когда супремум в $(2.36)$ достигается при $\lambda \rightarrow \infty$. Тогда возможна следующая альтернатива.

1) Существует последовательность $\left\{\lambda_{k}\right\}_{k=1}^{\infty}$ из локалъных максимумов такая, что

$$
\lambda_{k}^{\theta} G_{\lambda_{k}}(\xi, \xi) \rightarrow \sup _{\lambda>0}\left\{\lambda^{\theta} G_{\lambda}(\xi, \xi)\right\}
$$

Поскольку производная обращается в нуль в локальных максимумах, то, как и раньше, последовательность относительных экстремалей $u_{n}(x):=G_{\lambda_{n}}(\xi, x)$ не позволяет постоянной $K$ быть строго меньше, чем $K(\xi)$, и константа $(2.36)$ точная.

2) Функция $\lambda^{\theta} G_{\lambda}(\xi, \xi)$ в конечном счете монотонно возрастает при $\lambda \rightarrow \infty$. Тогда предел

$$
G_{\infty}:=\lim _{\lambda \rightarrow \infty} \lambda^{\theta} G_{\lambda}(\xi, \xi)
$$

существует и строго положителен. Поскольку производная интегрируема, то при $k \rightarrow \infty$

$$
0 \leftarrow \delta_{k}=\int_{2^{k}}^{2^{k+1}} \frac{d}{d \lambda}\left(\lambda^{\theta} f_{\xi}(\lambda)\right) d \lambda=\left.2^{k} \frac{d}{d \lambda}\left(\lambda^{\theta} f_{\xi}(\lambda)\right)\right|_{\lambda=\lambda_{k}}
$$

и для $\lambda_{k} \rightarrow \infty$ и $\varepsilon_{k}:=\delta_{k} \cdot 2^{-k} \lambda_{k} \rightarrow 0$ выполняется равенство

$$
\left.\frac{d}{d \lambda}\left(\lambda^{\theta} f_{\xi}(\lambda)\right)\right|_{\lambda=\lambda_{k}}=\varepsilon_{k} \lambda_{k}^{-1}
$$

Вместе с (2.38) это дает

$$
\lambda_{k}^{\theta} f_{\xi}\left(\lambda_{k}\right)=G_{\infty}+o_{\lambda \rightarrow \infty}(1), \quad \lambda_{k}^{1+\theta} f_{\xi}^{\prime}\left(\lambda_{k}\right)=-\theta G_{\infty}+o_{\lambda \rightarrow \infty}(1)
$$

Поэтому

$$
\frac{D\left(\lambda_{k}\right)}{\lambda_{k}}=\frac{1-\theta}{\theta}+o_{\lambda \rightarrow \infty}(1) .
$$

Наконец, для $u_{k}(x):=G_{\lambda_{k}}(\xi, x)$ после очевидных преобразований получаем

$$
\frac{\left|u_{k}(\xi)\right|^{2}}{\left\|u_{k}\right\|_{B}^{2 \theta}\left\|u_{k}\right\|_{A}^{2(1-\theta)}}=\lambda_{k}^{\theta} G_{\lambda_{k}} \cdot\left(\frac{\lambda_{k}}{D\left(\lambda_{k}\right)}\right)^{1-\theta}\left(1+\frac{D\left(\lambda_{k}\right)}{\lambda_{k}}\right) .
$$

Переходя к пределу $k \rightarrow \infty$ и используя (2.39), получаем справа $K(\xi)$, что доказывает, что и во втором случае $K(\xi)$ есть точная постоянная.

Осталось доказать, что если в $(2.35),(2.36)$ есть экстремальная функция $u_{*}$, то супремум по $\lambda$ в (2.36) достигается в конечной точке. 
Воспользуемся элементарным тождеством для положительных $a, b$ :

$$
\lambda_{*}^{\theta} a^{2(1-\theta)} b^{2 \theta}=\theta^{\theta}(1-\theta)^{(1-\theta)}\left(a^{2}+\lambda_{*} b^{2}\right), \quad \lambda_{*}=\frac{\theta}{1-\theta} \frac{a^{2}}{b^{2}} .
$$

Тогда для экстремальной функции $u_{*}$ получаем

$$
\begin{aligned}
u_{*}(\xi) & =K(\xi)\|u\|_{B}^{2 \theta}\|u\|_{A}^{2(1-\theta)}=K(\xi) \frac{\theta^{\theta}(1-\theta)^{(1-\theta)}}{\lambda_{*}^{\theta}}\left(\left\|u_{*}\right\|_{A}^{2}+\lambda_{*}\left\|u_{*}\right\|_{B}^{2}\right) \\
& =\sup _{\lambda>0}\left\{\lambda^{\theta} G_{\lambda}(\xi, \xi)\right\} \frac{1}{\lambda_{*}^{\theta}}\left(\left\|u_{*}\right\|_{A}^{2}+\lambda_{*}\left\|u_{*}\right\|_{B}^{2}\right), \quad \text { где } \lambda_{*}=\frac{\theta}{1-\theta} \frac{\left\|u_{*}\right\|_{A}^{2}}{\left\|u_{*}\right\|_{B}^{2}} .
\end{aligned}
$$

Лемма 2.1 теперь говорит, что в случае равенства обязательно имеем $u_{*}(x)=$ $G_{\lambda_{*}}(x, \xi)$, и

$$
\sup _{\lambda>0}\left\{\lambda^{\theta} G_{\lambda}(\xi, \xi)\right\} \frac{1}{\lambda_{*}^{\theta}}=G_{\lambda_{*}}(\xi, \xi), \quad \text { или } \sup _{\lambda>0}\left\{\lambda^{\theta} G_{\lambda}(\xi, \xi)\right\}=\lambda_{*}^{\theta} G_{\lambda_{*}}(\xi, \xi) .
$$

Теорема доказана.

ЗАмечАниЕ 2.1. В рассматриваемом компактном случае выполняется неравенство Пуанкаре (2.7). Поэтому неравенство (2.35) с точной постоянной (2.36) выполняется не только для $\theta=\theta_{0}:=(2 m-n) /(2(m-l))$, но и для всех $0 \leqslant \theta \leqslant \theta_{0}$ (когда $A$-норма имеет бо́льший вес). Действительно, доказательство слово в слово такое же, даже проще, поскольку для $\theta^{\prime}<\theta_{0}$ вместо $(2.38)$ имеем

$$
\lim _{\lambda \rightarrow \infty} \lambda^{\theta^{\prime}} G_{\lambda}(\xi, \xi)=0
$$

так что экстремальная функция всегда существует. Кроме того, при $\theta=0$ имеем

$$
\|u(\xi)\|^{2} \leqslant \sup _{\lambda>0} G_{\lambda}(\xi, \xi)\|u\|_{A}^{2}=G_{0}(\xi, \xi)\|u\|_{A}^{2},
$$

поскольку $G_{\lambda}(\xi, \xi)$ убывает по $\lambda($ см. $(2.26))$.

2.5. Асимптотическое разложение при больших $\lambda$. В этом разделе будут выведены полезные асимптотические формулы для функции $\mathbb{V}(\xi, D)$ при $D \rightarrow \infty$. Для этого мы должны знать асимптотическое поведение функции Грина $f_{\xi}(\lambda)=G_{\lambda}(\xi, \xi)$. Используя локализацию и метод замороженных коэффициентов, можно показать, что предел (2.38) существует и строго положителен, так что главный член асимптотического разложения $G_{\lambda}$ мы знаем. Однако дальнейшие члены асимптотики зависят от конкретной задачи, и мы не располагаем общей формулой для них. По этой причине мы просто предполагаем, что при $\lambda \rightarrow \infty$

$$
f_{\xi}(\lambda)=\lambda^{-1}\left(g_{1} \lambda^{1-\theta}+g_{2}+g_{3} \lambda^{\theta-1}+o\left(\lambda^{\theta-1}\right)\right),
$$

где $g_{1}=g_{1}(\xi)>0, g_{2}=g_{2}(\xi), g_{3}=g_{3}(\xi)$ - некоторые заданные числа (зависящие от $\xi$ ), и что разложение (2.41) можно продифференцировать по $\lambda$. Это предположение будет выполняться в большинстве рассматриваемых приложений. Тогда справедлив следующий результат. 
ПредлОЖЕНИЕ 2.1. Пусть выполнены предположения, перечисленные в разделе 2.1. Пусть справедливо асимптотическое разложение (2.41). Тогда для решения экстремальной задачи $\mathbb{V}(\xi, D)$ справедливо асимптотическое разложение при $D \rightarrow \infty$ :

$$
\begin{gathered}
\mathbb{V}(\xi, D)=g_{1} S D^{1-\theta}+g_{2} \frac{1}{\theta}-\frac{1}{2} S^{-1} \frac{g_{2}^{2}(1-\theta)-2 \theta g_{1} g_{3}}{\theta^{3} g_{1}} D^{\theta-1}+o\left(D^{\theta-1}\right), \\
S:=\frac{1}{\theta^{\theta}(1-\theta)^{1-\theta}} .
\end{gathered}
$$

ДокАЗАТЕЛЬство состоит в непосредственном (хотя и достаточно трудоемком) вычислении. Полагая для краткости $g_{1}=a, g_{2}=b, g_{3}=c$, имеем

$$
\begin{aligned}
& f(\lambda)=a \lambda^{-\theta}+b \lambda^{-1}+c \lambda^{-(2-\theta)}+o\left(\lambda^{-(2-\theta)}\right), \\
& g(\lambda)=a \theta \lambda^{-(1+\theta)}+b \lambda^{-2}+c(2-\theta) \lambda^{-(3-\theta)}+o\left(\lambda^{-(3-\theta)}\right), \\
& h(\lambda)=f(\lambda)-\lambda g(\lambda)=a(1-\theta) \lambda^{-\theta}-c(1-\theta) \lambda^{-(2-\theta)}+o\left(\lambda^{-(2-\theta)}\right) .
\end{aligned}
$$

Далее находим асимптотику при $D \rightarrow \infty$ единственного решения $\lambda=\lambda(D)$ первого уравнения в $(2.31)$ :

$$
\begin{aligned}
D(\lambda) & =\frac{h(\lambda)}{g(\lambda)}=\frac{\lambda\left(a(1-\theta)-c(1-\theta) \lambda^{-(2-2 \theta)}+\cdots\right)}{a \theta+b \lambda^{-(1-\theta)}+c(2-\theta) \lambda^{-(2-2 \theta)}+\cdots} \\
& =\frac{1-\theta}{\theta}\left(\lambda-\frac{b}{a \theta} \lambda^{\theta}+\frac{b^{2}-2 \theta a c}{a^{2} \theta^{2}} \lambda^{-1+2 \theta}+\cdots\right)
\end{aligned}
$$

или

$$
\lambda-A \lambda^{\theta}+B \lambda^{-1+2 \theta}+\cdots=\delta:=D \frac{\theta}{1-\theta}, \quad A=\frac{b}{a \theta}, \quad B=\frac{b^{2}-2 \theta a c}{a^{2} \theta^{2}} .
$$

Единственное большое решение этого уравнения имеет асимптотику при $\delta \rightarrow \infty$

$$
\lambda(\delta)=\delta+A \delta^{\theta}+C \delta^{-1+2 \theta}+\cdots,
$$

где $C$ находим, подставляя это выражение в (2.43), что дает

$$
C=\theta A^{2}-B=\frac{-b^{2}(1-\theta)+2 a c \theta}{a^{2} \theta^{2}},
$$

или, окончательно,

$$
\lambda(D)=r D+s D^{\theta}+t D^{2 \theta-1}+\cdots
$$

где

$$
r=\frac{\theta}{1-\theta}, \quad s=\frac{b}{a} \frac{1}{\theta^{1-\theta}(1-\theta)^{\theta}}, \quad t=\frac{2 a c \theta-(1-\theta) b^{2}}{a^{2} \theta^{3-2 \theta}(1-\theta)^{2 \theta-1}} .
$$

Остается подставить $(2.44)$ в $\mathbb{V}=f^{2} / g=D \cdot f^{2} / h$, где для $f^{2} / h$ имеется разложение

$$
\frac{f(\lambda)^{2}}{h(\lambda)}=\frac{a}{1-\theta} \lambda^{-\theta}+\frac{2 b}{1-\theta} \lambda^{-1}+\frac{b^{2}+3 a c}{(1-\theta) a} \lambda^{\theta-2}+\cdots .
$$


Для каждой степени из (2.44) получаем соответственно

$$
\begin{aligned}
\lambda(D)^{-\theta} & =\frac{1}{r^{\theta}} D^{-\theta}-\frac{\theta s}{r^{1+\theta}} D^{-1}+\frac{\theta(\theta+1) s^{2}-2 \theta t r}{2 r^{2+\theta}} D^{\theta-2}+\cdots \\
\lambda(D)^{-1} & =\frac{1}{r} D^{-1}-\frac{s}{r^{2}} D^{\theta-2}+\cdots \\
\lambda(D)^{\theta-2} & =\frac{1}{r^{2-\theta}} D^{\theta-2}+\cdots .
\end{aligned}
$$

Подставляя это в (2.45) и умножая на $D$, после нескольких чудесных сокращений получаем асимптотическое разложение (2.42). Предложение доказано.

ЗАмечАниЕ 2.2. В частности, если для некоторой точки $\xi \in \mathscr{M}$ третий член в $(2.42)$ отрицательный (а это всегда так при $\left.g_{3} \leqslant 0\right)$, то

$$
\mathbb{V}(\xi, D)<g_{1} S D^{1-\theta}+\frac{g_{2}}{\theta}
$$

при больших $D$. Тогда, используя вычисления на компьютере, которые являются достоверными на ограниченных промежутках изменения $D$, или аналитические оценки, мы покажем во второй части статьи, что в отдельных случаях неравенство (2.46) верно для всех значений $D$. Тогда из этого следует улучшенный вариант неравенства (2.9) с точными постоянными:

$$
|u(\xi)|^{2} \leqslant g_{1} S\|u\|_{B}^{\theta}\|u\|_{A}^{1-\theta}+\frac{g_{2}}{\theta}\|u\|_{B}^{2} .
$$

Отметим также интересный факт, что

$$
D^{\prime}(\lambda)=\left(\frac{f_{\xi}(\lambda)+\lambda f_{\xi}^{\prime}(\lambda)}{-f_{\xi}^{\prime}(\lambda)}\right)^{\prime}=\frac{2\left(f_{\xi}^{\prime}(\lambda)\right)^{2}-f_{\xi}^{\prime \prime}(\lambda) f(\lambda)}{\left(f_{\lambda}^{\prime}(\xi)\right)^{2}}
$$

и поэтому положительность $D^{\prime}(\lambda)$, установленная в лемме 2.2, эквивалентна строгой вогнутости функции $\lambda \mapsto 1 / f_{\xi}(\lambda)$.

2.6. Вариационное представление $\mathbb{V}(\xi, \lambda)$. В этом разделе приводится простое, но очень полезное представление функции $\mathbb{V}(\xi, \lambda)$ в терминах функции Грина $G_{\lambda}(\xi, \xi)$, не содержащее производных по $\lambda$ и позволяющее во многих случаях аналитически доказать справедливость неравенства (2.46) для всех допустимых значений $D$.

Теорема 2.3. Если выполнены условия теоремы 2.1, то функиия $\mathbb{V}(\xi, D)$, определенная в (2.29), допускает следующее вариационное представление:

$$
\mathbb{V}(\xi, D)=\inf _{\lambda \in\left[-\lambda_{0}, \infty\right)}\left\{(\lambda+D) G_{\lambda}(\xi, \xi)\right\}
$$

где $D \in\left[\lambda_{0}, \infty\right)$, а функиия Грина $G_{\lambda}(\xi, \xi)$ определена в (2.14). 
ДоказатЕЛьСтво. Прежде всего, из полученных ранее результатов следует, что функция $\lambda \mapsto(\lambda+D) G_{\lambda}(\xi, \xi)$ стремится к $+\infty$, когда $\lambda \rightarrow \infty$ или $\lambda \rightarrow-\lambda_{0}$, для любого $D \in\left(\lambda_{0}, \infty\right)$. Таким образом, инфимум в $(2.48)$ достигается в некоторой точке $\lambda_{*}=\lambda_{*}(D)$ внутри этого интервала. Ясно, что в этой точке $\lambda_{0}$

$G_{\lambda}(\xi, \xi)+(D+\lambda) \frac{d}{d \lambda} G_{\lambda}(\xi, \xi)=0, \quad$ или $D=-\frac{\lambda \frac{d}{d \lambda} G_{\lambda}(\xi, \xi)+G_{\lambda}(\xi, \xi)}{\frac{d}{d \lambda} G_{\lambda}(\xi, \xi)}=\frac{h_{\xi}(\lambda)}{g_{\xi}(\lambda)}$

(см. $(2.30),(2.31)$ и $(2.32))$. Таким образом, уравнение для $\lambda_{*}(D)$ совпадает с уравнением (2.19) для $\lambda(D)$, которое имеет единственное решение, и поэтому минимум в $(2.48)$ достигается точно в $\lambda_{*}=\lambda(D)$. Остается проверить, что значение в этой точке и есть $\mathbb{V}(\xi, \lambda)$. С этой целью заметим, что числа $D$ и $\lambda$ удовлетворяют первому уравнению в (2.31), поэтому, используя второе уравнение в (2.31), получаем

$$
(\lambda+D) G_{\lambda}(\xi, \xi)=\frac{f_{\lambda}(\xi)\left(h_{\xi}(\lambda)+\lambda g_{\xi}(\lambda)\right)}{g_{\xi}(\lambda)}=\frac{f_{\xi}(\lambda)^{2}}{g_{\xi}(\lambda)}=\mathbb{V}(\xi, D) .
$$

Теорема доказана.

2.7. Обобщения. Здесь мы кратко обсудим возможности ослабления предположений о многообразии $\mathscr{M}$ и операторах $A$ и $B$.

I) Операторы $A$ и $B$ - не обязательно эллиптические дифференииалъные операторы. Вся теория слово в слово работает, если мы предположим, что $A$ и $B-$ эллиптические псевдодифференциальные операторы (например, $A=(-\Delta)^{m}$ и $B=(-\Delta)^{l}$, где $\Delta$ - оператор Лапласа-Бельтрами на $\left.\mathscr{M}\right)$, и, в частности, числа $m$ и $l$ не обязательно целые. Это даст возможность изучать интерполяционные неравенства в пространствах Соболева дробного порядка.

II) Разработанная теория естественным образом распространяется на случай многообразия с краем. Тогда необходимо снабдить эллиптические операторы $A$ и $B$ подходящими краевыми условиями, которые должны дополнительно удовлетворять условию

$$
\mathscr{D}(A) \subset \mathscr{D}(B)
$$

где $\mathscr{D}(A)$ и $\mathscr{D}(B)$ - области определения операторов $A$ и $B$. При этих дополнительных предположениях развитая выше теория работает и для многообразий с краем.

III) Можно рассмотреть сужение пространств $\bar{H}^{m}$ на класс, скажем, радиально симметричных функций. Если операторы $A$ и $B$ тоже радиально симметричны, то вся теория также работает и в этом случае.

IV) Разработанная теория работает во многих случаях, когда $\mathscr{M}$ некомпактно, точнее, неограничено. Единственная проблема здесь в том, что в отличие от компактного случая функция Грина $G_{\lambda}(x, \xi)$ может иметь плохое поведение при $x, \xi \rightarrow \infty$ и в результате использовавшиеся выше интегралы могут не иметь смысла. Так что в общей теории следует налагать дополнительные условия 
на $\mathscr{M}$, равно как и на операторы $A$ и $B$. Однако во всех наших "некомпактных" приложениях эти вопросы будут очевидными и прозрачными, так что, для того чтобы избежать ненужных технических усложнений, мы не будем здесь развивать “общую теорию” для некомпактного случая.

\section{3. Часть II. Примеры и приложения}

3.1. Случай $\mathbb{R}^{n}$. Для иллюстрации нашего метода рассмотрим простейший случай, когда $M=\mathbb{R}^{n}$, и пусть $l$ и $m$ удовлетворяют неравенству $-\infty<$ $l<n / 2<m<\infty$, так что $0<\theta=(2 m-n) /(2(m-l))<1$.

Теорема 3.1. Справедливо неравенство

$$
\|u\|_{\infty}^{2} \leqslant c_{\mathbb{R}^{n}}(l, m)\left\|(-\Delta)^{l / 2} u\right\|^{2 \theta}\left\|(-\Delta)^{m / 2} u\right\|^{2(1-\theta)},
$$

где точная постоянная $c_{\mathbb{R}^{n}}(l, m)$ есть

$c_{\mathbb{R}^{n}}(l, m)=(2 \pi)^{-n} \int_{\mathbb{R}^{n}} \frac{d \xi}{\theta|\xi|^{2 l}+(1-\theta)|\xi|^{2 m}}=\frac{\sigma(n) \pi}{(2 \pi)^{n} \cdot 2(m-l) \theta^{\theta}(1-\theta)^{1-\theta} \sin \pi \theta}$.

ДокАЗАтЕльство. Для $\widehat{f}(\xi)=\mathscr{F}(f)(\xi)=(2 \pi)^{-n / 2} \int_{\mathbb{R}^{n}} e^{-i \xi x} f(x) d x$ и $\lambda>0$ имеем

$$
\begin{aligned}
\|u\|_{\infty}^{2} \leqslant & (2 \pi)^{-n}\left(\int_{\mathbb{R}^{n}}|\widehat{u}(\xi)| d \xi\right)^{2} \\
\leqslant & (2 \pi)^{-n} \int_{\mathbb{R}^{n}}|\widehat{u}(\xi)|^{2}\left(\lambda \theta|\xi|^{2 l}+(1-\theta)|\xi|^{2 m}\right) d \xi \int_{\mathbb{R}^{n}} \frac{d \xi}{\lambda \theta|\xi|^{2 l}+(1-\theta)|\xi|^{2 m}} \\
= & (2 \pi)^{-n} \int_{\mathbb{R}^{n}} \frac{d \xi}{\lambda \theta|\xi|^{2 l}+(1-\theta)|\xi|^{2 m}} \\
& \times\left(\lambda \theta\left\|(-\Delta)^{l / 2} u\right\|^{2}+(1-\theta)\left\|(-\Delta)^{m / 2} u\right\|^{2}\right) .
\end{aligned}
$$

Далее, полагая $\lambda=\lambda_{*}=\left\|(-\Delta)^{m / 2} u\right\|^{2} /\left\|(-\Delta)^{l / 2} u\right\|^{2}$, получаем

$$
\lambda_{*} \theta\left\|(-\Delta)^{l / 2} u\right\|^{2}+(1-\theta)\left\|(-\Delta)^{m / 2} u\right\|^{2}=\lambda_{*}^{\theta}\left\|(-\Delta)^{l / 2} u\right\|^{2 \theta}\left\|(-\Delta)^{m / 2} u\right\|^{2(1-\theta)},
$$

что дает

$$
\|u\|_{\infty}^{2} \leqslant(2 \pi)^{-n}\left[\lambda_{*}^{\theta} \int_{\mathbb{R}^{n}} \frac{d \xi}{\lambda_{*} \theta|\xi|^{2 l}+(1-\theta)|\xi|^{2 m}}\right]\left\|(-\Delta)^{l / 2} u\right\|^{2 \theta}\left\|(-\Delta)^{m / 2} u\right\|^{2(1-\theta)} .
$$

Выражение в квадратных скобках на самом деле не зависит от $\lambda_{*}$, что и дает (3.2). Тот факт, что постоянная точная, можно проверить, подставляя

$$
u_{\lambda}(x)=\mathscr{F}^{-1}\left(\left(\lambda \theta_{1}|\xi|^{2 l}+\theta_{2}|\xi|^{2 m}\right)^{-1}\right)
$$

и вычисляя соответствующие интегралы. Однако более простой способ состоит в том, чтобы заметить, что для $u_{\lambda}(x)$ все неравенства в (3.3) обращаются в равенства. Далее, дифференцируя выражение в квадратных скобках по $\lambda$, получаем $\lambda=\left\|(-\Delta)^{l / 2} u_{\lambda}\right\|^{2} /\left\|(-\Delta)^{m / 2} u_{\lambda}\right\|^{2}$, что и доказывает, что (3.1) для $u=u_{\lambda}$ превращается в равенство. Теорема доказана. 
ЗАмечАниЕ 3.1. Конечно, тот же самый результат получается применением теоремы 2.1. Тогда

$$
\mathbb{A}(\lambda)=(-\Delta)^{m}+\lambda(-\Delta)^{l}, \quad G_{\lambda}(x, \xi)=\frac{1}{(2 \pi)^{n}} \int_{\mathbb{R}^{n}} \frac{e^{i \eta(x-\xi)} d \eta}{|\eta|^{2 m}+\lambda|\eta|^{2 l}}
$$

И

$$
G_{\lambda}(\xi, \xi)=\lambda^{-\theta} \frac{\sigma(n) \pi}{(2 \pi)^{n} \cdot 2(m-l) \sin \pi \theta} .
$$

СлЕДСТвИЕ 3.1. Для любого D>0 экстремальная задача

$$
\mathbb{V}(D):=\sup \left\{|u(0)|^{2}:\left\|(-\Delta)^{l / 2} u\right\|^{2}=1,\left\|(-\Delta)^{m / 2} u\right\|^{2}=D\right\}
$$

имеет решение

$$
\mathbb{V}(D)=c_{\mathbb{R}^{n}}(l, m) D^{1-\theta}
$$

Единственная экстремальная функиия есть (см. (3.5))

$$
U_{D}=\frac{u_{D}}{\left\|(-\Delta)^{l / 2} u_{D}\right\|}
$$

ЗАмЕчАниЕ 3.2. Для вычисления интеграла была использована формула

$$
\int_{0}^{\infty} \frac{x^{m}}{\left(1+x^{k}\right)^{l}} d x=\frac{1}{k} \mathrm{~B}\left(\frac{m+1}{k}, l-\frac{m+1}{k}\right),
$$

которая будет полезна и в дальнейшем.

ЗАмечАниЕ 3.3. В одномерном случае постоянная $c_{\mathbb{R}}(l, m)$ найдена в [24].

3.2. Симметрические многообразия. В этом разделе рассматривается случай, когда исходное многообразие $\mathscr{M}$ симметрическое, а операторы $A$ и $B$ инвариантны по отношению к группе симметрий. Как следствие, функция Грина $G_{\lambda}(x, \xi)$, определенная в $(2.14)$, зависит только от $x-\xi$ (поскольку любые две точки на $\mathscr{M}$ могут быть отождествлены с помощью подходящего преобразования симметрии). Поэтому ключевая функция $G_{\lambda}(\xi, \xi)$ на самом деле не зависит от $\xi$ и является функцией лишь одной переменной $\lambda$ :

$$
G_{\lambda}(\xi, \xi)=G(\lambda)
$$

Этот факт чрезвычайно упрощает анализ и позволяет во многих случаях получать явные выражения для точных констант в интерполяционных неравенствах. Ниже рассматриваются два примера: торы и сферы, хотя развитая техника применима и для других симметрических многообразий.

3.2.1. Тор. Мы начнем с рассмотрения $n$-мерного тора $\mathbb{T}^{n}:=[-\pi, \pi]^{n}$ и соответствующих неравенств вида

$$
\|u\|_{L_{\infty}\left(\mathbb{T}^{n}\right)} \leqslant C_{l, n, m}\left\|(-\Delta)^{l / 2} u\right\|_{L_{2}\left(\mathbb{T}^{n}\right)}^{\theta}\left\|(-\Delta)^{m / 2} u\right\|_{L_{2}\left(\mathbb{T}^{n}\right)}^{1-\theta}
$$


для периодических функций $u \in H^{m}\left(\mathbb{T}^{n}\right)$ со средним $\langle u\rangle:=\int_{\mathbb{T}^{n}} u(x) d x=0$. В этом случае $A:=(-\Delta)^{m}, B=(-\Delta)^{l}$, $\operatorname{ker} A=\operatorname{ker} B=\{$ const $\}$ и все предположения развитой выше абстрактной теории выполнены при $l<n / 2<m$. В частности,

$$
\bar{H}^{m}=H^{m}\left(\mathbb{T}^{n}\right) \cap\{\langle u\rangle=0\} .
$$

Функция Грина оператора $\mathbb{A}(\lambda)=(-\Delta)^{m}+\lambda(-\Delta)^{l}$ на торе $\mathbb{T}^{n}$ (с условием нулевого среднего) записывается с помощью кратных рядов Фурье:

$$
\begin{aligned}
& G_{\lambda}(x, \xi)=\frac{1}{(2 \pi)^{n}} \sum_{k \in \mathbb{Z}_{0}^{n}} \frac{e^{i k(x-\xi)}}{|k|^{2 m}+\lambda|k|^{2 l}}, \\
& G_{\lambda}(\xi, \xi)=G(\lambda)=\frac{1}{(2 \pi)^{n}} \sum_{k \in \mathbb{Z}_{0}^{n}} \frac{1}{|k|^{2 m}+\lambda|k|^{2 l}},
\end{aligned}
$$

где $k=\left(k_{1}, \ldots, k_{n}\right)$ - мультииндекс, $|k|^{2}=k_{1}^{2}+\cdots+k_{n}^{2}$ и сумма берется по всем $k=\left(k_{1}, \ldots, k_{n}\right) \neq(0, \ldots, 0)$ и $\mathbb{Z}_{0}^{n}=\mathbb{Z}^{n} \backslash\{0\}$.

3.2.1.1. Случай $l=0$. Конкретный случай $l=0$ был изучен в [2]. Тогда асимптотическое разложение $G(\lambda)$ при $\lambda \rightarrow \infty$ получается с помощью формулы суммирования Пуассона (см., например, [22]):

$$
\sum_{m \in \mathbb{Z}^{n}} f\left(\frac{m}{\mu}\right)=(2 \pi)^{n / 2} \mu^{n} \sum_{m \in \mathbb{Z}^{n}} \widehat{f}(2 \pi m \mu),
$$

где $\mathscr{F}(f)(\xi)=\widehat{f}(\xi)=(2 \pi)^{-n / 2} \int_{\mathbb{R}^{n}} f(x) e^{-i \xi x} d x-$ преобразование Фурье и $\mu>0$.

А именно,

$$
\Phi_{\lambda}(x)=(2 \pi)^{-n / 2} \mathscr{F}^{-1}\left(\frac{1}{|\xi|^{2 m}+\lambda}\right)=\frac{1}{(2 \pi)^{n}} \int_{\mathbb{R}^{n}} \frac{e^{i \xi x} d \xi}{|\xi|^{2 m}+\lambda}
$$

есть фундаментальное решение оператора $\mathbb{A}(\lambda)$ во всем пространстве $\mathbb{R}^{n}$. Тогда из (3.13) следует, что

$$
G(\lambda)+\frac{1}{(2 \pi)^{n}} \lambda^{-1}=\frac{1}{(2 \pi)^{n}} \sum_{k \in \mathbb{Z}^{n}} \frac{1}{|k|^{2 m}+\lambda}=\sum_{k \in \mathbb{Z}^{n}} \Phi_{\lambda}(2 \pi k) .
$$

Кроме того, в силу масштабной инвариантности имеем

$$
\Phi_{\lambda}(x)=\lambda^{-1} \lambda^{n /(2 m)} \Phi_{1}\left(\lambda^{1 /(2 m)} x\right)
$$

и в силу аналитичности функции $\xi \mapsto 1 /\left(|\xi|^{2 m}+1\right)$ получаем $\left|\Phi_{1}(x)\right| \leqslant C e^{-C_{m}|x|}$ для некоторых положительных $C$ и $C_{m}$. Поэтому из (3.14) следует, что

$$
G(\lambda)=\lambda^{-1} \lambda^{n /(2 m)} \Phi_{1}(0)-\frac{1}{(2 \pi)^{n}} \lambda^{-1}+O\left(e^{-C_{m} \lambda^{1 /(2 m)}}\right) .
$$

Наконец, вычисляя с помощью (3.8) интеграл $\Phi_{1}(0)=(2 \pi)^{-n} \int_{\mathbb{R}^{n}} \frac{d \xi}{|\xi|^{2 m}+1}$, находим

$$
G(\lambda)=(2 \pi)^{-n} \lambda^{-1}\left(\frac{\pi \sigma(n)}{2 m \sin (\pi n /(2 m))} \lambda^{n /(2 m)}-1\right)+O\left(e^{-C_{m} \lambda^{1 /(2 m)}}\right),
$$


где $\sigma(n)=2 \pi^{n / 2} / \Gamma(n / 2)$ - площадь поверхности $(n-1)$-мерной единичной сферы. Поскольку $1-\theta=n /(2 m)$, то асимптотическая формула (3.16) имеет вид $(2.41)$ с $g_{3}=0$ и из предложения 2.1 получаем

$$
\mathbb{V}(D)=c_{n}(m) D^{n /(2 m)}-k_{n}(m)-l_{n}(m) D^{-n /(2 m)}+O\left(D^{-n / m}\right),
$$

где

$$
\begin{aligned}
c_{n}(m) & :=c_{\mathbb{R}^{n}}(0, m)=\frac{\pi \sigma(n)}{(2 \pi)^{n} n^{n /(2 m)}(2 m-n)^{1-n /(2 m)} \sin (\pi n /(2 m))}, \\
k_{n}(m) & :=\frac{2 m}{(2 \pi)^{n}(2 m-n)} \\
l_{n}(m) & :=\frac{2 n^{1+n /(2 m)} m^{2} \sin (\pi n /(2 m))}{(2 \pi)^{n} \pi \sigma(n)(2 n-m)^{2+n /(2 m)}}
\end{aligned}
$$

(см. подробнее в [2]). Итак, поскольку $l_{n}(m)>0$, то

$$
\mathbb{V}(D) \leqslant c_{n}(m) D^{n /(2 m)}-k_{n}(m)
$$

для больших $D$, и, используя непрерывность функции $D \mapsto \mathbb{V}(D)$, заключаем, что

$$
K_{n}(m):=\sup _{D \in[1, \infty)}\left\{c_{n}(m) D^{n /(2 m)}-\mathbb{V}(D)\right\}<\infty
$$

и

$$
\mathbb{V}(D) \leqslant c_{n}(m) D^{n /(2 m)}-K_{n}(m)
$$

теперь уже для всех $D \geqslant 1$. Тогда согласно (2.11) получаем следующий результат из [2].

Теорема 3.2. Для всех $u \in \bar{H}^{m}\left(\mathbb{T}^{n}\right)$ справедливо неравенство

$$
\|u\|_{L_{\infty}\left(\mathbb{T}^{n}\right)}^{2} \leqslant c_{n}(m)\|u\|_{L_{2}\left(\mathbb{T}^{n}\right)}^{2-n / m}\left\|(-\Delta)^{m / 2} u\right\|_{L_{2}\left(\mathbb{T}^{n}\right)}^{n / m}-K_{n}(m)\|u\|_{L^{2}\left(\mathbb{T}^{n}\right)}^{2},
$$

где постоянные $c_{n}(m)$ и $K_{n}(m) \leqslant k_{n}(m)$ точные и определены в (3.18) $и$ (3.20) соответственно.

Отсюда видно, что постоянная $c_{n}(m)$ здесь совпадает с точной постоянной в аналогичном неравенстве во всем $\mathbb{R}^{n}(3.2)$. Напротив, точная постоянная $K_{n}(m)$ вряд ли имеет аналитическое выражение для всех $n$ и $m$, поскольку в силу определения (3.20), для того чтобы получить $K_{n}(m)$, мы должны знать функцию $V(D)$ не только для больших $D$, а для всех $D \geqslant 1$. Как показано в [2], асимптотическое разложение (3.17) работает только для оченъ болъших $D$ (если $m$ очень велико) и на начальном интервале $D$ эта функция осииллирует (что может быть объяснено исследованием предела $m \rightarrow \infty$, см. [2]).

Кроме того, существует принципиальная разница между случаем $n=1$ и многомерным случаем $n>1$. В первом случае [2] всегда $K_{1}(m)>0$, так что дополнительный член в (3.22) улучшает классическое интерполяционное неравенство. Однако в многомерном случае эта постоянная становится строго отрицательной при достаточно больших $m$ (например, при $m>9$ для $n=2$ 
и при $m>6$ для $n=3)$. Поэтому в этом случае дополнительный член положителен и необходим для выполнения интерполяционного неравенства. Другими словами, если нас интересует только классическое интерполяционное неравенство (без корректоров), то необходимо увеличить постоянную по сравнению со случаем $\mathbb{R}^{n}$.

Тем не менее, как показано в [2] с помощью достоверных вычислений, есть три конкретных случая, когда выполняется равенство $K_{n}(m)=k_{n}(m)$, и, следовательно, все постоянные в (3.22) могут быть найдены аналитически.

1) Случай $n=1$ и $m=1$ : неравенство

$$
\|u\|_{L_{\infty}\left(\mathbb{T}^{1}\right)}^{2} \leqslant\|u\|_{L_{2}\left(\mathbb{T}^{1}\right)}\left\|u^{\prime}\right\|_{L_{2}\left(\mathbb{T}^{1}\right)}-\frac{1}{\pi}\|u\|_{L_{2}\left(\mathbb{T}^{1}\right)}^{2}
$$

выполняется для всех $2 \pi$-периодических функций с нулевым средним.

2) Случай $n=1$ и $m=2$ : неравенство

$$
\|u\|_{L_{\infty}\left(\mathbb{T}^{1}\right)}^{2} \leqslant \frac{\sqrt{2}}{\sqrt[4]{27}}\|u\|_{L_{2}\left(\mathbb{T}^{1}\right)}^{3 / 2}\left\|u^{\prime \prime}\right\|_{L_{2}\left(\mathbb{T}^{1}\right)}^{1 / 2}-\frac{2}{3 \pi}\|u\|_{L_{2}\left(\mathbb{T}^{1}\right)}^{2}
$$

выполняется для всех $2 \pi$-периодических функций с нулевым средним.

3) Случай $n=2$ и $m=2$ : неравенство

$$
\|u\|_{L_{\infty}\left(\mathbb{T}^{2}\right)}^{2} \leqslant \frac{1}{4}\|u\|_{L_{2}\left(\mathbb{T}^{2}\right)}\|\Delta u\|_{L_{2}\left(\mathbb{T}^{2}\right)}-\frac{1}{2 \pi^{2}}\|u\|_{L_{2}\left(\mathbb{T}^{2}\right)}^{2}
$$

выполняется для всех $(2 \pi \times 2 \pi)$-периодических функций с нулевым средним. Как показывают вычисления, это единственный случай (по крайней мере для целого $m$ ) для двумерного тора, когда $K_{n}(m)=k_{n}(m)$, см. [2].

Используя развитую выше технику, мы сейчас приведем чисто аналитическое доказательство двух первых неравенств.

Теорема 3.3. Неравенства (3.23) и (3.24) выполняются для всех $2 \pi$-периодических функиий с нулевым средним, и все константы в этих неравенствах точные.

ДокАЗАтЕЛЬСтво. Начнем с (3.23). Поскольку

$$
\Phi_{\lambda}(x)=(2 \pi)^{-1 / 2} \mathscr{F}^{-1}\left(\frac{1}{\xi^{2}+\lambda}\right)=\frac{1}{2 \sqrt{\lambda}} e^{-\sqrt{\lambda}|x|},
$$

то, используя (3.14) и суммируя геометрическую прогрессию, находим

$$
G(\lambda)=\sum_{k \in \mathbb{Z}} \frac{1}{2 \sqrt{\lambda}} e^{-2 \pi \sqrt{\lambda}|k|}-\frac{1}{2 \pi \lambda}=\frac{1}{2 \pi} \frac{\pi \sqrt{\lambda} \operatorname{coth}(\pi \sqrt{\lambda})-1}{\lambda} .
$$

По теореме 2.3 , для $D \geqslant 1$

$$
\mathbb{V}(D)=\min _{\lambda \geqslant-1}\{(\lambda+D) G(\lambda)\} \leqslant\left.\{(\lambda+D) G(\lambda)\}\right|_{\lambda=D-1 / 2}=\left(2 D-\frac{1}{2}\right) G\left(D-\frac{1}{2}\right),
$$


где минимум по $\lambda$ заменен значением в $\lambda=D-1 / 2$. Итак, осталось только доказать, что

$$
\begin{gathered}
\left(2 D-\frac{1}{2}\right) G\left(D-\frac{1}{2}\right)-D^{1 / 2}+\frac{1}{\pi}=\frac{1}{4 \pi(2 D-1)}(4 \pi D \sqrt{4 D-2} \cdot \operatorname{coth} \alpha \\
\left.-\pi \sqrt{4 D-2} \cdot \operatorname{coth} \alpha-2-8 \pi D^{3 / 2}+4 \pi \sqrt{D}\right)<0
\end{gathered}
$$

для всех $D \geqslant 1$, где для краткости обозначено $\alpha:=\pi \sqrt{4 D-2} / 2$. Для упрощения правой части (3.19) используем неравенство

$$
\sqrt{4 D-2}<2 \sqrt{D}-\frac{1}{2} D^{-1 / 2}-\frac{1}{16} D^{-3 / 2}, \quad D \geqslant 1
$$

(которое следует из тейлоровского разложения функции $\sqrt{1-(2 D)^{-1}}$ и того факта, что все отбрасываемые члены отрицательны). Используя также, что $\operatorname{coth} \alpha \geqslant 1$ в отрицательных членах, получаем

$$
\begin{aligned}
\mathbb{V}(D)- & D^{1 / 2}+\frac{1}{\pi} \leqslant \frac{1}{4 \pi(2 D-1)}\left(4 \pi D\left(8 D^{1 / 2}-2 D^{-1 / 2}-\frac{D^{-3 / 2}}{4}\right) \operatorname{coth} \alpha\right. \\
& \left.-\pi \sqrt{4 D-2} \cdot \operatorname{coth} \alpha-2-8 \pi D^{3 / 2}+4 \pi \sqrt{D}\right) \\
= & \frac{1}{16 \pi D^{1 / 2}(2 D-1)}\left(32 \pi D^{2}(\operatorname{coth} \alpha-1)\right. \\
& -8 \pi D \operatorname{coth} \alpha-\pi \operatorname{coth} \alpha-4 \pi \sqrt{4 D-2} \sqrt{D}-8 \sqrt{D}+16 \pi D) \\
< & \frac{1}{16 \pi D^{1 / 2}(2 D-1)}\left(\frac{64 \pi D^{2}}{e^{2 \alpha}-1}-8 \pi D-\pi-4 \pi \sqrt{4 D-2} \sqrt{D}-8 \sqrt{D}+16 \pi D\right) \\
= & \frac{1}{16 \pi D^{1 / 2}(2 D-1)}\left(\frac{64 \pi D^{2}}{\exp (\pi \sqrt{4 D-2})-1}+\frac{4 \pi}{1+\sqrt{1-(2 D)-1}}-\pi-8 \sqrt{D}\right) .
\end{aligned}
$$
Функция $f(D):=\frac{64 \pi D^{2}}{\exp (\pi \sqrt{4 D-2})-1}$ строго убывает при $D \geqslant 1$, поскольку
производная

$$
f^{\prime}(D)=-\frac{128 \pi D(\sqrt{4 D-2}-\sqrt{4 D-2} \exp (\pi \sqrt{4 D-2})+\pi D \exp (\pi \sqrt{4 D-2}))}{(-1+\exp (\pi \sqrt{4 D-2}))^{2} \sqrt{4 D-2}}
$$

строго отрицательна при $D \geqslant 1$. Аналогично, второе и последнее слагаемые справа в (3.29) строго убывают, и заменяя их на максимальное значение в $D=1$, окончательно получаем

$$
\begin{aligned}
\mathbb{V}(D)-D^{1 / 2}+\frac{1}{\pi} & <\frac{1}{16 \pi D^{1 / 2}(2 D-1)}\left(\frac{64 \pi}{\exp (\pi \sqrt{2})-1}+\frac{8 \pi}{2+\sqrt{2}}-\pi-8\right) \\
& =\frac{1}{16 \pi D^{1 / 2}(2 D-1)}(-1.3873 \ldots)<0
\end{aligned}
$$


что и доказывает неравенство (3.23). То, что все константы точные, следует из полученной ранее асимптотики (3.17):

$$
\mathbb{V}(D)=D^{1 / 2}-\frac{1}{\pi}+O\left(D^{-1 / 2}\right) \quad \text { при } D \rightarrow \infty .
$$

Заметим, что общая формула (2.44) в нашем случае дает $\lambda(D)=D-(2 / \pi) \sqrt{D}+$ $\cdots$. Годятся и другие подстановки $\lambda$ в (3.27), например, $\lambda=D-1, \lambda=D-$ $(2 / \pi) \sqrt{D}$. Однако $\lambda=D$ не годится.

Теперь рассмотрим (3.24). Суммируя ряд с помощью вычетов (см. [25; раздел 3.3]):

$$
\sum_{n=1}^{\infty} \frac{1}{\mu^{4}+n^{4}}=\frac{\pi \sqrt{2}}{4 \mu^{3}} \frac{\sinh (\pi \sqrt{2} \mu)+\sin (\pi \sqrt{2} \mu)}{\cosh (\pi \sqrt{2} \mu)-\cos (\pi \sqrt{2} \mu)}-\frac{1}{2 \mu^{4}}
$$

находим

$$
G(\lambda)=\frac{2}{2 \pi} \sum_{n=1}^{\infty} \frac{1}{n^{4}+\lambda}=\frac{1}{\pi}\left(\frac{\pi \sqrt{2}}{4 \lambda^{3 / 4}} \frac{\sinh \left(\pi \sqrt{2} \lambda^{1 / 4}\right)+\sin \left(\pi \sqrt{2} \lambda^{1 / 4}\right)}{\cosh \left(\pi \sqrt{2} \lambda^{1 / 4}\right)-\cos \left(\pi \sqrt{2} \lambda^{1 / 4}\right)}-\frac{1}{2 \lambda}\right) .
$$

Поскольку

$$
\frac{\sinh \alpha+\sin \alpha}{\cosh \alpha-\cos \alpha} \leqslant \frac{\sinh \alpha+1}{\cosh \alpha-1}<1+4.1 e^{-\alpha}
$$

для $\alpha=\pi \sqrt{2} \lambda^{1 / 4} \geqslant \pi \sqrt{2}=4.4428 \ldots$, то

$$
G(\lambda)<G_{1}(\lambda):=\frac{1}{\pi}\left(\frac{\pi \sqrt{2}}{4 \lambda^{3 / 4}}\left(1+4.1 e^{-\pi \sqrt{2} \lambda^{1 / 4}}\right)-\frac{1}{2 \lambda}\right) .
$$

Используя теорему 2.3 и подставляя $\lambda=3 D-3 / 2$, получаем

$$
\begin{aligned}
\mathbb{V}(D) & =\min _{\lambda \geqslant-1}\{(\lambda+D) G(\lambda)\} \leqslant\left.\{(\lambda+D) G(\lambda)\}\right|_{\lambda=3(D-1 / 2)} \\
& =\left(4 D-\frac{3}{2}\right) G\left(3 D-\frac{3}{2}\right)<\left(4 D-\frac{3}{2}\right) G_{1}\left(3 D-\frac{3}{2}\right) .
\end{aligned}
$$

Поэтому достаточно показать, что

$$
R(D):=\left(4 D-\frac{3}{2}\right) G_{1}\left(3 D-\frac{3}{2}\right)-\frac{\sqrt{2}}{\sqrt[4]{27}} D^{1 / 4}+\frac{2}{3 \pi} \leqslant 0 .
$$

Полагая $x:=(3 D-3 / 2)^{1 / 4}, \quad x \geqslant(3 / 2)^{1 / 4}=1.1066 \ldots$, имеем

$$
\begin{aligned}
6 \pi R(D)= & \left(8 x^{4}+3\right)\left(\frac{\pi \sqrt{2}}{4 x^{3}}\left(1+4.1 e^{-\pi \sqrt{2} x}\right)-\frac{1}{2 x^{4}}\right)-2 \sqrt{2} \pi\left(x^{4}+\frac{3}{2}\right)^{1 / 4}+4 \\
= & 2 \pi \sqrt{2}\left(\left(x+\frac{3}{8 x^{3}}-\left(x^{4}+\frac{3}{2}\right)^{1 / 4}\right)+\left(x+\frac{3}{8 x^{3}}\right) 4.1 e^{-\pi \sqrt{2} x}\right) \\
& \quad-\frac{3}{2 x^{4}}=: R(x) .
\end{aligned}
$$


Первый член в скобках порядка $O\left(x^{-7}\right)$, второй экспоненциально мал, поэтому $R(x)<0$ уже при $x \geqslant 2$. Далее, $R(x)<0$ и на интервале $x \in\left[(3 / 2)^{1 / 4}, 2\right]$, в чем можно убедится непосредственно. Итак, $R(x)<0$ для всех $x$.

В заключение заметим, что общая формула (2.44) дает

$$
\lambda(D)=3 D-\frac{4 \sqrt{2}}{\pi \sqrt[4]{3}} D^{3 / 4}+\cdots,
$$

что и объясняет (по крайней мере в главном члене) использованную подстановку $\lambda=3 D-3 / 2$. Теорема доказана.

3.2.1.2. Случай $l>0$. Анализ функции Грина (3.12) в случае $l>0$ более тонкий. Действительно, теперь функция $k \rightarrow 1 /\left(|k|^{2 m}+\lambda|k|^{2 l}\right)$ имеет особенность при $k=0$. Поэтому соответствующее фундаментальное решение $\Phi_{\lambda}(x)$ не является быстро убывающим и прямое применение формулы Пуассона не дает желаемого результата. Для преодоления этого обстоятельства, следуя [2], мы положим $\mu=\lambda^{-1}$ и перепишем (3.14) в виде

$$
G(\lambda)=(2 \pi)^{-n} \mu \sum_{k \in \mathbb{Z}_{0}^{n}} \frac{1}{k^{2 l}\left(\mu|k|^{2(m-l)}+1\right)}=:(2 \pi)^{-n} \mu \widetilde{G}(\mu) .
$$

Тогда, $s$ раз дифференцируя $\widetilde{G}(\mu)$ по $\mu$, получаем

$$
\frac{d^{s} \widetilde{G}(\mu)}{d \mu^{s}}=(-1)^{s} s ! \sum_{k \in \mathbb{Z}_{0}^{n}} \frac{|k|^{2(m-l) s-2 l}}{\left(\mu|k|^{2(m-l)}+1\right)^{s+1}}
$$

и функция $k \mapsto|k|^{2(m-l) s-2 l} /\left(\mu|k|^{2(m-l)}+1\right)^{s+1}$ уже регулярна при $k=0$, если $s \geqslant l /(m-l)$ (а убывание по $k \rightarrow \infty$ остается порядка $|k|^{2 m}$ для всех $s$ ). Теперь мы можем применить формулу Пуассона для того, чтобы выяснить (аналогично (3.14)) асимптотическое поведение функции (3.33) при $\mu \rightarrow 0+$. Затем после $s$ интегрирований мы получим асимптотику исходной функции $G(\lambda)$ c точностъю до s постоянных интегрирования, которые каким-то образом нужно найти, см. ниже.

Мы проиллюстрируем этот метод для конкретного случая $l=1$ и $m=2$. При этом рассмотрим лишь двумерный $(n=2)$ и трехмерный $(n=3)$ случаи, хотя общий случай рассматривается аналогично.

Пусть $n=2$. Тогда $\widetilde{G}(\mu)$ надо продифференцировать только один раз, чтобы убрать особенность при $k=0$ :

$$
\frac{d \widetilde{G}(\mu)}{d \mu}=\frac{d}{d \mu} \sum_{k \in \mathbb{Z}_{0}^{2}} \frac{1}{|k|^{2}\left(1+\mu|k|^{2}\right)}=-\sum_{k \in \mathbb{Z}_{0}^{2}} \frac{1}{\left(1+\mu|k|^{2}\right)^{2}} .
$$

Применяя формулу Пуассона, с учетом равенства $\mathscr{F}\left(\left(1+|x|^{2}\right)^{-2}\right)=|\xi| K_{1}(|\xi|) / 2$ получаем

$$
\frac{d \widetilde{G}(\mu)}{d \mu}=-\frac{\pi}{\mu}+1-2 \pi^{2} \mu^{-3 / 2} \sum_{k \in \mathbb{Z}_{0}^{2}}|k| K_{1}\left(2 \pi \mu^{-1 / 2}|k|\right),
$$


где $K_{n}(z)$ - модифицированная функция Бесселя порядка $n$ [27]. Интегрируя этот ряд по $\mu$ и используя формулу $K_{0}^{\prime}(x)=-K_{1}(x)$, получаем нужную формулу

$$
\widetilde{G}(\mu)=\pi \ln \frac{1}{\mu}+\beta+\mu-2 \pi \sum_{k \in \mathbb{Z}_{0}^{2}} K_{0}\left(2 \pi \mu^{-1 / 2}|k|\right),
$$

где $\beta$ - постоянная интегрирования. Как показано в [2], на основании формулы Харди

$$
\sum_{k \in \mathbb{Z}_{0}^{2}} \frac{1}{|k|^{2 s}}=4 \zeta(1+s) \beta(1+s)
$$

где $\zeta(s)$ и $\beta(s)$ - дзета-функция Римана и бета-функция Дирихле, для постоянной интегрирования получается аналитическое выражение

$$
\beta=\pi \gamma+4 \beta^{\prime}(1)=\pi\left(2 \gamma+2 \ln 2+3 \ln \pi-4 \ln \Gamma\left(\frac{1}{4}\right)\right),
$$

где $\gamma$ - постоянная Эйлера-Маскерони, а Г $(z)$ - гамма-функция Эйлера. Таким образом, получен следующий результат.

Лемма 3.1. Пусть $n=2, m=2, l=1$. Тогда для $\lambda>0$ функиия Грина (3.32) имеет следующее представление:

$$
\begin{aligned}
G(\lambda) & =\frac{1}{4 \pi} \lambda^{-1} \ln \lambda+\frac{\beta}{4 \pi^{2} \lambda}+\frac{1}{4 \pi^{2} \lambda^{2}}-\frac{1}{2 \pi} \lambda^{-1} \sum_{k \in \mathbb{Z}_{0}^{2}} K_{0}\left(2 \pi \lambda^{1 / 2}|k|\right) \\
& \leqslant \frac{1}{4 \pi^{2} \lambda}\left(\pi \ln \lambda+\beta+\frac{1}{\lambda}\right)
\end{aligned}
$$

где $\beta$ определена в (3.37). Кроме того, член, содержащий сумму бесселевых функций в (3.38), экспоненциально мал (порядка $\left.O\left(e^{-\pi \lambda^{1 / 2}}\right)\right)$ при $\lambda \rightarrow \infty$.

Заметим, что случай $n=2$ и $m=2, l=1$ есть предельный случай в $(2.9)$, в котором появляется дополнительный логарифмический член, и соответствующее неравенство обычно называется неравенством Брезиса-Галлуэ (см. [4]). Хотя условие (2.8) формально и нарушается, большая часть развитой выше теории работает и в этом предельном случае. В частности, как показано в [2] на основе (3.38), существует постоянная $L>0$ такая, что

$$
\mathbb{V}(D) \leqslant \frac{1}{4 \pi}(\ln D+\ln (1+\ln D)+L), \quad D \geqslant 1,
$$

где постоянная $1 /(4 \pi)$ точная, и численно найденное значение $L$ равно 2.15627. Это дает улучшенный вариант неравенства Брезиса-Галлуэ в форме

$$
\|u\|_{C\left(\mathbb{T}^{2}\right)}^{2} \leqslant \frac{1}{4 \pi}\|\nabla u\|_{L^{2}\left(\mathbb{T}^{2}\right)}^{2}\left(\ln \frac{\|\Delta u\|_{L^{2}\left(\mathbb{T}^{2}\right)}^{2}}{\|\nabla u\|_{L^{2}\left(\mathbb{T}^{2}\right)}^{2}}+\ln \left(1+\ln \frac{\|\Delta u\|_{L^{2}\left(\mathbb{T}^{2}\right)}^{2}}{\|\nabla u\|_{L^{2}\left(\mathbb{T}^{2}\right)}^{2}}\right)+L\right) .
$$


Рассмотрим теперь трехмерный случай $n=3$ с $m=2$ и $l=1$. Тогда

$$
\widetilde{G}(\mu)=\sum_{k \in \mathbb{Z}_{0}^{3}} \frac{1}{|k|^{2}\left(1+\mu|k|^{2}\right)}, \quad \frac{d \widetilde{G}(\mu)}{d \mu}=-\sum_{k \in \mathbb{Z}_{0}^{3}} \frac{1}{\left(1+\mu|k|^{2}\right)^{2}} .
$$

Применяя формулу суммирования Пуассона (3.13) ко второму ряду справа и вычисляя преобразование Фурье, а именно, используя формулу [22]

$$
\mathscr{F}\left(\frac{1}{\left(1+|x|^{2}\right)^{2}}\right)(\xi)=\frac{\pi^{2}}{(2 \pi)^{3 / 2}} e^{-|\xi|},
$$

окончательно получаем

$$
\frac{d \widetilde{G}(\mu)}{d \mu}=1-\frac{\pi^{2}}{\mu^{3 / 2}} \sum_{k \in \mathbb{Z}^{3}} e^{-2 \pi \mu^{-1 / 2}|k|^{1 / 2}} .
$$

Интегрируя по $\mu$ и выделяя в сумме член с $k=0$, получаем

$$
\widetilde{G}(\mu)=2 \pi^{2} \mu^{-1 / 2}+\beta_{3}+\mu-\pi \sum_{k \in \mathbb{Z}_{0}^{3}}|k|^{-1 / 2} e^{-2 \pi \mu^{-1 / 2}|k|^{1 / 2}},
$$

где $\beta_{3}$ - постоянная интегрирования, которая может быть найдена численно:

$$
\beta_{3}=-8.91363291758515127
$$

(ниже показано, как эффективно ее вычислять). Итак, доказана следующая лемма.

Лемма 3.2. Пусть $n=3, m=2, l=1$. Тогда при $\lambda>0$ функция Грина (3.32) может быть записана в виде

$$
\begin{aligned}
G(\lambda) & =\frac{1}{8 \pi^{3} \lambda}\left(2 \pi^{2} \lambda^{1 / 2}+\beta_{3}+\lambda^{-1}-\pi \sum_{k \in \mathbb{Z}_{0}^{3}} \frac{e^{-2 \pi \lambda^{1 / 2}|k|^{1 / 2}}}{|k|^{1 / 2}}\right) \\
& \leqslant \frac{1}{8 \pi^{3} \lambda}\left(2 \pi^{2} \lambda^{1 / 2}+\beta_{3}+\lambda^{-1}\right),
\end{aligned}
$$

где $\beta_{3}$ определена в (3.37). Кроме того, член с суммой в середине (3.45) экспоненииально мал (порядка $\left.O\left(e^{-\pi \lambda^{1 / 2}}\right)\right)$ при $\lambda \rightarrow \infty$.

Таким образом, функция Грина $G(\lambda)$ удовлетворяет условиям предложения 2.1 с $\theta=1 / 2$ и $\left(g_{1}, g_{2}, g_{3}\right)=\left(8 \pi^{3}\right)^{-1} \cdot\left(2 \pi^{2}, \beta_{3}, 1\right)$ соответственно. Поэтому

$$
\mathbb{V}(D)=\frac{1}{8 \pi^{3}}\left(4 \pi^{2} D^{1 / 2}+2 \beta_{3}-\frac{\beta_{3}^{2}-4 \pi^{2}}{2 \pi^{2}} D^{-1 / 2}\right)+O\left(D^{-1}\right),
$$

где третий член отрицательный, что говорит о том, что может быть верно следующее неравенство:

$$
\|u\|_{\infty}^{2} \leqslant \frac{1}{2 \pi}\|\nabla u\|_{L_{2}\left(\mathbb{T}^{3}\right)}\|\Delta u\|_{L_{2}\left(\mathbb{T}^{3}\right)}-\frac{-\beta_{3}}{4 \pi^{3}}\|\nabla u\|_{L_{2}\left(\mathbb{T}^{3}\right)}^{2},
$$

где все постоянные точные. Однако на данный момент мы доказали это неравенство лишь для больших $D=\|\Delta u\|^{2} /\|\nabla u\|^{2}$. В следующей лемме показывается, что оно верно для всех $D \geqslant 1$. 
Лемма 3.3. Неравенство (3.47) верно для любого $и \in H^{2}\left(\mathbb{T}^{3}\right)$ с нулевым средним.

ДокАзАтельство. Нам лишь надо проверить, что неравенство

$$
\mathbb{V}(D) \leqslant \frac{1}{8 \pi^{3}}\left(4 \pi^{2} D^{1 / 2}+2 \beta_{3}\right)
$$

выполняется для всех $D \geqslant 1$. Для этого мы вновь используем вариационное представление (3.27), где полагаем $\lambda=D-1 / 2$, а также неравенство (3.45). Выделяя член $4 \pi^{2} D^{1 / 2}+2 \beta_{3}$, получаем

$$
\begin{aligned}
8 \pi^{3} \mathbb{V}(D) \leqslant( & \left.+\frac{D}{D-1 / 2}\right)\left(2 \pi^{2} \sqrt{D-\frac{1}{2}}+\beta_{3}+\frac{1}{D-1 / 2}\right)=4 \pi^{2} D^{1 / 2}+2 \beta_{3} \\
& +\frac{1}{2 D-1}\left(\pi^{2} \frac{\sqrt{4 D-2}}{\sqrt{4 D(4 D-2)}+4 D-1}+\beta_{3}+4+\frac{2}{2 D-1}\right),
\end{aligned}
$$

и надо проверить, что второй член в правой части всегда отрицательный. Действительно, при $D \geqslant 1$

$$
\begin{aligned}
\pi^{2} \frac{\sqrt{4 D-2}}{\sqrt{4 D(4 D-2)}+4 D-1} & <\pi^{2} \frac{\sqrt{4 D-2}}{\sqrt{4 D(4 D-2)}+4 D-2} \\
& =\pi^{2} \frac{1}{\sqrt{4 D}+\sqrt{4 D-2}} \leqslant \frac{\pi^{2}}{2+\sqrt{2}}
\end{aligned}
$$

и $2 /(2 D-1) \leqslant 2$. Тогда

$$
\frac{\pi^{2}}{2+\sqrt{2}}+\beta_{3}+6=-0.022892<0
$$

и лемма доказана.

3.2.1.3. Нахождение постоянных интегрирования. Как мы уже видели, при $l>0$ формула суммирования Пуассона позволяет найти асимптотическое разложение функции Грина $G(\lambda)$ лишь с точностью до некоторых постоянных интегрирования. Прямое вычисление этих постоянных является нетривиальной задачей, поскольку ряд (3.12) сходится недостаточно быстро, особенно для больших $\lambda$. Поэтому для их вычисления хотелось бы найти ряд, сходящийся более быстро (например, экспоненциально). В этом пункте для рассмотренного выше случая $n=3, m=2, l=1$ мы приводим явную формулу для функции Грина (3.12) в терминах интегралов от так называемых тэта-функций Якоби. Используя известные соотношения для тэта-функций, мы получаем формулу для $\beta_{3}$ в терминах очень быстро сходящегося и удобного для вычислений ряда. Заметим также, что хотя мы и ограничились этим конкретным случаем, предлагаемый метод имеет общий характер и применим для вычисления других постоянных интегрирования, включая случай анизотропных торов и т. д. 
Рассмотрим тэта-функцию Якоби (см., например, [8])

$$
\theta_{3}(q)=\sum_{k \in \mathbb{Z}} q^{k^{2}}
$$

Справедливо известное тождество, играющее ключевую роль в дальнейшем:

$$
\theta_{3}\left(e^{-\pi t}\right)=\frac{1}{\sqrt{t}} \theta_{3}\left(e^{-\pi t^{-1}}\right)
$$

(это тождество вытекает из формулы (3.13) с $n=1, f(x)=e^{-x^{2} / 2}, \widehat{f}(\xi)=e^{-\xi^{2} / 2}$ и $\mu=\sqrt{t /(2 \pi)})$.

Используя очевидное равенство

$$
\frac{1}{k(1+\mu k)}=\int_{0}^{\infty}\left(1-e^{-t / \mu}\right) e^{-k t} d t
$$

преобразуем (3.41) следующим образом:

$$
\begin{aligned}
8 \pi^{3} \widetilde{G}(\mu) & =\int_{0}^{\infty}\left(1-e^{-t / \mu}\right) \sum_{k \in \mathbb{Z}_{0}^{3}}\left[e^{-t}\right]^{k_{1}^{2}}\left[e^{-t}\right]^{k_{2}^{2}}\left[e^{-t}\right]^{k_{3}^{2}} d t \\
& =\int_{0}^{\infty}\left(1-e^{-t / \mu}\right)\left(\left[\theta_{3}\left(e^{-t}\right)\right]^{3}-1\right) d t
\end{aligned}
$$

Разбивая интервал интегрирования, $\mathbb{R}_{+}=[0,1] \cup[1, \infty)$, и используя $(3.51)$, приходим к

$$
\begin{aligned}
8 \pi^{3} \widetilde{G}(\mu)= & \int_{1}^{\infty}\left(1-e^{-t / \mu}\right)\left(\left[\theta_{3}\left(e^{-t}\right)\right]^{3}-1\right) d t \\
& +\int_{0}^{1}\left(1-e^{-t / \mu}\right)\left(\pi^{3 / 2} t^{-3 / 2} \theta_{3}\left(e^{-\pi^{2} t^{-1}}\right)^{3}-1\right) d t \\
= & \int_{1}^{\infty}\left(1-e^{-t / \mu}\right)\left(\left[\theta_{3}\left(e^{-t}\right)\right]^{3}-1\right) d t \\
& +\pi^{3 / 2} \int_{1}^{\infty}\left(1-e^{-1 /(t \mu)}\right) t^{-1 / 2}\left(\theta_{3}\left(e^{-\pi^{2} t}\right)^{3}-1\right) d t \\
& \quad-\int_{0}^{1}\left(1-e^{-t / \mu}\right) d t+\pi^{3 / 2} \int_{0}^{1} t^{-3 / 2}\left(1-e^{-t / \mu}\right) d t
\end{aligned}
$$

Теперь можно найти асимптотическое разложение $\widetilde{G}(\mu)$ при $\mu \rightarrow 0+$. Несложные вычисления дают

$$
\begin{aligned}
\int_{0}^{1} t^{-3 / 2}\left(1-e^{-t / \mu}\right) d t & =\mu^{-1 / 2}\left(\int_{0}^{\infty} t^{-3 / 2}\left(1-e^{-t}\right) d t-\int_{1 / \mu}^{\infty} t^{-3 / 2}\left(1-e^{-t}\right) d t\right) \\
& =\mu^{-1 / 2}\left(2 \sqrt{\pi}-\int_{1 / \mu}^{\infty} t^{-3 / 2} d t+O\left(e^{-1 / \mu}\right)\right) \\
& =2 \sqrt{\pi} \mu^{-1 / 2}-2+o_{\mu}(1) .
\end{aligned}
$$


Учитывая, что $\theta_{3}(x)-1=O(x)$ при $x \rightarrow 0$, мы можем перейти к пределу $\mu \rightarrow 0$ в интегралах в $(3.52)$, содержащих $\theta_{3}$, и, сравнивая получившийся результат с (3.44), находим

$$
\beta_{3}=-1-2 \pi^{3 / 2}+\int_{1}^{\infty}\left(\theta_{3}\left(e^{-t}\right)^{3}-1\right) d t+\pi^{3 / 2} \int_{1}^{\infty} t^{-1 / 2}\left(\theta_{3}\left(e^{-\pi^{2} t}\right)^{3}-1\right) d t .
$$

Мы не знаем, можно ли сосчитать интегралы в (3.54) в явном виде, однако полученная формула очень удобна для вычисления $\beta_{3}$ с высокой точностью. Действительно, возвращаясь к ряду Тейлора (3.50) для тэта-функции и используя равенство

$$
\int_{1}^{\infty} t^{-1 / 2} e^{-\alpha t} d t=\sqrt{\pi} \frac{\operatorname{erfc}(\sqrt{\alpha})}{\sqrt{\alpha}}, \quad \alpha>0,
$$

где $\operatorname{erfc}(x):=\frac{2}{\sqrt{\pi}} \int_{x}^{\infty} e^{-t^{2}} d t$, получаем

$$
\beta_{3}=-1-2 \pi^{3 / 2}+\sum_{k \in \mathbb{Z}_{0}^{3}}\left(\frac{e^{-|k|^{2}}}{|k|^{2}}+\pi \frac{\operatorname{erfc}(\pi|k|)}{|k|}\right) .
$$

Видим, что скорость сходимости ряда сверхэкспоненциальная, и вычисления дают значение

$$
\beta_{3}=-8.91363291758515127,
$$

использованное в предыдущем пункте.

3.2.2. Неравенства на сферах. Напомним основные факты, касающиеся спектра оператора Лапласа-Бельтрами на $(d-1)$-мерной сфере $\mathbb{S}^{d-1}$ :

$$
-\Delta Y_{n}^{k}=\Lambda_{n} Y_{n}^{k}, \quad k=1, \ldots, k_{d}(n), \quad n=1,2, \ldots .
$$

Здесь $Y_{n}^{k}$ - это ортонормированные сферические гармоники. Каждое собственное значение

$$
\Lambda_{n}=n(n+d-2)
$$

имеет кратность

$$
k_{d}(n)=\frac{2 n+d-2}{n}\left(\begin{array}{c}
n+d-3 \\
n-1
\end{array}\right) .
$$

В частности, для $d=3,4$ имеем

$$
\begin{array}{ll}
\mathbb{S}^{2}: \Lambda_{n}=n(n+1), & k_{3}(n)=2 n+1, \\
\mathbb{S}^{3}: \Lambda_{n}=n(n+2), & k_{4}(n)=(n+1)^{2} .
\end{array}
$$

Справедливо следующее тождество [22], играющее в дальнейшем ключевую роль: для любого $\xi \in \mathbb{S}^{d-1}$

$$
\sum_{l=1}^{k_{d}(n)} Y_{n}^{l}(\xi)^{2}=\frac{k_{d}(n)}{\sigma(d)}
$$

где $\sigma(d)=2 \pi^{d / 2} / \Gamma(d / 2)$ - площадь поверхности $\mathbb{S}^{d-1}$. 
Наконец, поскольку ядро $\Delta$ одномерно и состоит из констант, всюду в дальнейшем предполагается ортогональность константам:

$$
\bar{H}^{s}\left(\mathbb{S}^{d-1}\right)=\left\{\varphi \in H^{s}\left(\mathbb{S}^{d-1}\right),(\varphi, 1)=0\right\}
$$

3.2.2.1. Неравенства на сферах: $\mathbb{S}^{2}$. Мы рассмотрим приложения общей теории к неравенствам на двумерной сфере с $A=(-\Delta)^{m}, m>1 / 2$, и $B=I$, где $\Delta$ - оператор Лапласа-Бельтрами. Первое положительное собственное значение $-\Delta$ есть 2 , и в соответствии с (2.13) мы рассмотрим оператор

$$
\mathbb{A}(\lambda)=(-\Delta)^{m}+\lambda I, \quad \lambda>-2^{m} .
$$

Его функция Грина $G_{\lambda}(x, \xi)$ есть

$$
G_{\lambda}(x, \xi)=\sum_{n=1}^{\infty} \sum_{k=1}^{2 n+1} \frac{Y_{n}^{k}(\xi) Y_{n}^{k}(x)}{(n(n+1))^{m}+\lambda}, \quad x, \xi \in \mathbb{S}^{2},
$$

и, благодаря $(3.57)$, функция $G_{\lambda}(\xi, \xi)$ не зависит от $\xi$ и задается рядом

$$
G(\lambda)=G_{\lambda}(\xi, \xi)=\sum_{n=1}^{\infty} \sum_{k=1}^{2 n+1} \frac{Y_{n}^{k}(\xi)^{2}}{(n(n+1))^{m}+\lambda}=\frac{1}{4 \pi} \sum_{n=1}^{\infty} \frac{2 n+1}{(n(n+1))^{m}+\lambda}
$$

Полагая $\mu:=\lambda^{-1 / m}$, получаем

$$
G(\lambda)=\frac{1}{4 \pi} \mu^{m} \sum_{n=1}^{\infty}(2 n+1) \varphi(\mu n(n+1))
$$

где

$$
\varphi(x)=\frac{1}{x^{m}+1}
$$

Поскольку $\varphi(0)=1$ и $\varphi^{\prime}(0)=0$, из приводимой ниже леммы 3.5 следует, что

$$
G(\lambda)=\frac{1}{4 \pi}\left[\lambda^{-\theta} K-\frac{2}{3} \lambda^{-1}+0 \cdot \lambda^{-(2-\theta)}\right]+O\left(\lambda^{-(3-2 \theta)}\right),
$$

где $\theta=(m-1) / m$ и

$$
K=\int_{0}^{\infty} \varphi(x) d x=\frac{(1-\theta) \pi}{\sin \theta \pi}
$$

Другими словами, мы показали, что для $f_{\xi}(\lambda)=G(\lambda)$ справедливо асимптотическое разложение $(2.41)$ с $g_{1}, g_{2}, g_{3}$, не зависящими от $\xi \in \mathbb{S}^{2}$ :

$$
g_{1}=\frac{K}{4 \pi}=\frac{1-\theta}{4 \sin \theta \pi}, \quad g_{2}=-\frac{1}{6 \pi}, \quad g_{3}=0 .
$$


Далее, дифференцируя (3.58) и вновь используя лемму 3.5, находим асимптотическое разложение $g_{\xi}(\lambda)=g(\lambda)$ :

$$
\begin{aligned}
g(\lambda) & =-f^{\prime}(\lambda)=-G^{\prime}(\lambda)=\frac{1}{4 \pi} \sum_{n=1}^{\infty} \frac{2 n+1}{\left((n(n+1))^{m}+\lambda\right)^{2}} \\
& =\frac{1}{4 \pi} \mu^{2 m} \sum_{n=1}^{\infty} \frac{2 n+1}{\left((\mu n(n+1))^{m}+1\right)^{2}} \\
& =\frac{1}{4 \pi}\left[\lambda^{-(1-\theta)} \theta K-\frac{2}{3} \lambda^{-2}+0 \cdot \lambda^{-(3-\theta)}\right]+O\left(\lambda^{-(4-\theta)}\right),
\end{aligned}
$$

где использовано равенство

$$
\int_{0}^{\infty} \varphi(x)^{2} d x=\int_{0}^{\infty} \frac{d x}{\left(x^{m}+1\right)^{2}}=\theta K
$$

Этим обосновано дифференцирование асимптотической формулы для $f(\lambda)$, как требуется в предложении 2.1, откуда получаем асимптотическое разложение функции $\mathbb{V}(D)$ на $\mathbb{S}^{2}$.

Теорема 3.4. Функиия $\mathbb{V}(D)$, являющаяся решением на $\mathbb{S}^{2}$ экстремальной задачи

$$
\mathbb{V}(\xi, D):=\sup \left\{|u(\xi)|^{2}: u \in \bar{H}^{m}\left(\mathbb{S}^{2}\right),\|u\|^{2}=1,\left\|(-\Delta)^{m / 2} u\right\|^{2}=D\right\},
$$

не зависит от $\xi \in \mathbb{S}^{2}$ и имеет следующее асимптотическое поведение при $D \rightarrow \infty:$

$$
\mathbb{V}(D)=\frac{1}{4 \sin \theta \pi}\left(\frac{1-\theta}{\theta}\right)^{\theta} D^{1-\theta}-\frac{1}{6 \pi \theta}-\frac{\sin \theta \pi}{18 \pi^{2}} \frac{(1-\theta)^{1-\theta}}{\theta^{3-\theta}} D^{\theta-1}+O\left(D^{-(2-2 \theta)}\right),
$$

где $\theta=(m-1) / m$.

Теперь используем полученную выше асимптотику функции $\mathbb{V}(D)$ для неравенств с остаточными членами на $\mathbb{S}^{2}$. По теореме 2.2 точная постоянная $K=$ $K_{\theta}$ в классическом интерполяционном неравенстве

$$
u(\xi)^{2} \leqslant\|u\|_{\infty}^{2} \leqslant K_{\theta}\|u\|^{2 \theta}\left\|(-\Delta)^{m} u\right\|^{2(1-\theta)}
$$

есть

$$
K_{\theta}=\frac{1}{\theta^{\theta}(1-\theta)^{1-\theta}} \sup _{\lambda \geqslant 0} \lambda^{\theta} G(\lambda)=\frac{1}{\theta^{\theta}(1-\theta)^{1-\theta}} \sup _{\lambda \geqslant 0} \frac{1}{4 \pi} \lambda^{\theta} \sum_{n=1}^{\infty} \frac{2 n+1}{(n(n+1))^{m}+\lambda} .
$$

Заметим, что выражение (3.64) было получено в [14] с помощью похожих, но менее общих методов, чем использованные нами в теореме 2.2. В [14] также было показано, что при (целых) $m, 2 \leqslant m \leqslant 7$, супремум достигается на бесконечности:

$$
\sup _{\lambda \geqslant 0} \lambda^{\theta} G(\lambda)=\lim _{\lambda \rightarrow \infty} \lambda^{\theta} G(\lambda)=\int_{0}^{\infty} \frac{d x}{1+x^{m}},
$$


что дает

$$
K_{\theta}=\frac{1}{4 \sin \theta \pi}\left(\frac{1-\theta}{\theta}\right)^{\theta}
$$

или, эквивалентно,

$$
\mathbb{V}(D)=\mathbb{V}_{m}(D)<\frac{1}{4 \sin \theta \pi}\left(\frac{1-\theta}{\theta}\right)^{\theta} D^{1-\theta}
$$

для $2 \leqslant m \leqslant 7$. Однако для бо́льших $m$ супремум в (3.65) достигается в конечной точке $\lambda_{*}<\infty$. Объяснение этому явлению для тора было дано в [2]; ниже мы сделаем это для сферы $\mathbb{S}^{2}$.

Лемма 3.4. Для всех достаточно больиих $m$ функиия $h(\lambda):=\lambda^{\theta} G(\lambda)$ достигает глобального максимума в конечной точке $\lambda_{*}=\lambda_{*}(m)$ u $h\left(\lambda_{*}\right)>h(\infty)$.

ДокАзАТЕЛьСтво. Полагая в (3.64) $\lambda=\nu^{2 m}$, получаем

$$
h(\lambda)=\frac{1}{4 \pi} H(\nu), \quad H(\nu)=\nu^{2 m-2} \sum_{n=1}^{\infty} \frac{2 n+1}{\nu^{2 m}+(n(n+1))^{m}} .
$$

Рассмотрим разбиение полуоси $x \geqslant 0$ точками

$$
a_{n}=a_{n}(\nu)=\frac{(n-1) n}{\nu^{2}}, \quad n=1, \ldots
$$

Тогда непосредственная проверка показывает, что

$$
H(\nu)=\frac{1}{2} \varphi\left(a_{2}\right)\left(a_{2}-a_{1}\right)+\sum_{n=2}^{\infty} \frac{\varphi\left(a_{n}\right)+\varphi\left(a_{n+1}\right)}{2}\left(a_{n+1}-a_{n}\right),
$$

где $\varphi(x)=1 /\left(1+x^{m}\right)$. Для больших $m$ эта функция выглядит как ступенька: $\varphi(x) \approx 1$ при $x<1$ и $\varphi(x) \approx 0$ при $x>1$. В силу приводимой ниже леммы 3.5

$$
H(\infty):=\lim _{\nu \rightarrow \infty} H(\nu)=\int_{0}^{\infty} \varphi(x) d x=\frac{\pi / m}{\sin (\pi / m)}=1+o_{m \rightarrow \infty}(1) .
$$

Зафиксируем большое $m$, и пусть, скажем, $\nu=\nu_{0}=\sqrt{2}+1 / 100$. Тогда

$$
\begin{gathered}
a_{1}\left(\nu_{0}\right)=0, \quad a_{2}\left(\nu_{0}\right)<1(=0.986), \quad a_{3}\left(\nu_{0}\right) \approx 3(=2.958) \\
a_{2}\left(\nu_{0}\right)-a_{1}\left(\nu_{0}\right)=0.986 ; \quad a_{3}\left(\nu_{0}\right)-a_{2}\left(\nu_{0}\right)=1.972 .
\end{gathered}
$$

Поскольку $\varphi\left(a_{2}\right)=1+o_{m \rightarrow \infty}(1), \varphi\left(a_{3}\right)=o_{m \rightarrow \infty}(1)$ и сумма от $n=3$ до $\infty$ в (3.67) дает вклад порядка $o_{m \rightarrow \infty}(1)$, то

$$
H\left(\nu_{0}\right)=\frac{0.986}{2}+\frac{1.972}{2}+o_{m \rightarrow \infty}(1)=1.479+o_{m \rightarrow \infty}(1) .
$$

Поэтому $H\left(\nu_{0}\right)>H(\infty)$, и лемма доказана. 

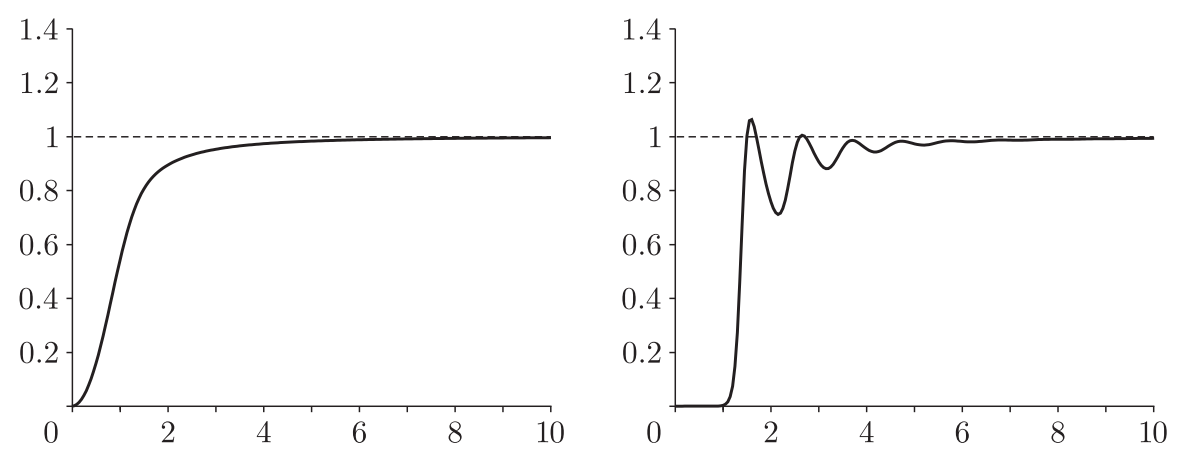

Рис. 1. Графики нормированных функций $H(\nu) / H(\infty)$ при $m=2$ и $m=10$

Это рассуждение также объясняет начальные осцилляции функции $H(\nu)$, когда соответствующий узел $a_{n}(\nu)$ проходит через 1 с увеличением $\nu$. На рис. 1 показано монотонное поведение $H(\nu)$ при $m=2$ и начальные осцилляции $H(\nu)$ при $m=10$. Заметим, что первый (и глобальный) максимум во втором случае расположен около $\nu=\sqrt{2}$.

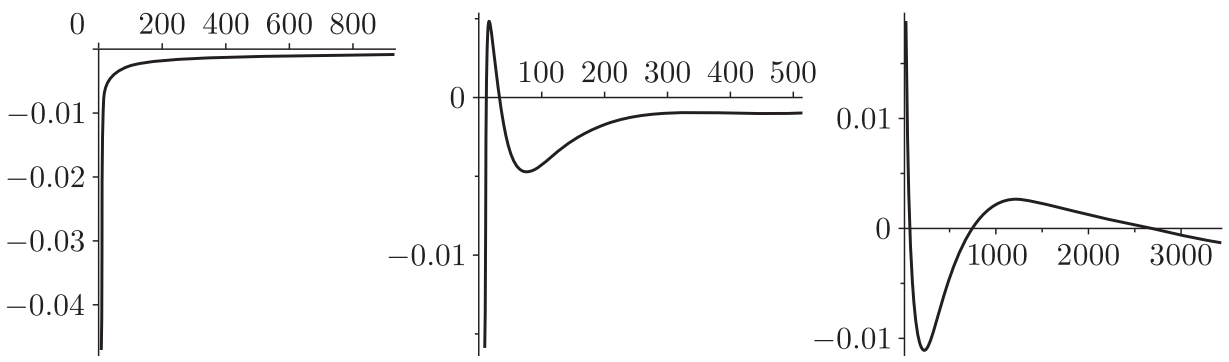

Рис. 2. График функции $\mathbb{V}(D)-\overline{\mathbb{V}}(D)$ при $m=2,3,4$

Переходя к неравенствам с остаточными членами, заметим, что третий член в (3.62) отрицательный, поэтому неравенство

$$
\mathbb{V}(D)<\frac{1}{4 \sin \theta \pi}\left(\frac{1-\theta}{\theta}\right)^{\theta} D^{1-\theta}-\frac{1}{6 \pi \theta}=: \overline{\mathbb{V}}(D)
$$

выполняется для всех $D \geqslant D_{0}$, где $D_{0}$ достаточно велико. На конечном интервале $\left[2^{m}, D_{0}\right]$ компьютерные вычисления достоверны, и их результаты приведены на рис. 2.

В частности, при $m=2$

$$
\mathbb{V}(D)<\overline{\mathbb{V}}(D) \text { для всех } D,
$$

что доказывает следующую теорему.

Tеорема 3.5. Если $u \in \bar{H}^{2}\left(\mathbb{S}^{2}\right)$, mo

$$
\|u\|_{\infty}^{2} \leqslant \frac{1}{4}\|u\|\|\Delta u\|-\frac{1}{3 \pi}\|u\|^{2},
$$

где обе постоянные точные, а экстремальной функции нет. 
При $m=3$ и $m=4$ функция $\mathbb{V}_{m}(\xi, D)-\overline{\mathbb{V}}_{m}(\xi, D)$ достигает глобального максимума $0.00486 \ldots$ при $D=15.8 \ldots$ для $m=3$ и глобального максимума $0.0189 \ldots$ при $D=22.4 \ldots$ для $m=4$. Соответственно получаем два точных неравенства

$$
\|u\|_{\infty}^{2} \leqslant \frac{1}{4 \sin \theta \pi}\left(\frac{1-\theta}{\theta}\right)^{\theta}\|u\|^{2 \theta}\left\|(-\Delta)^{m / 2} u\right\|^{2(1-\theta)}-\frac{\varepsilon_{m}}{6 \pi \theta}\|u\|^{2}, \quad m=3,4,
$$

где $\theta=(m-1) / m$ и $\varepsilon_{3}=0.938 \ldots, \varepsilon_{4}=0.821 \ldots$.

Теперь мы докажем асимптотическую формулу для функций, заданных рядом (3.71), которую мы систематически использовали выше. Итак, пусть функция $F(\mu)$ есть

$$
F(\mu):=\sum_{n=1}^{\infty}(2 n+1) f(\mu n(n+1)),
$$

где $f$ - достаточно гладкая и достаточно быстро убывающая функция. Требуется найти асимптотическое разложение $F(\mu)$ при $\mu \rightarrow 0$.

ЛЕмма 3.5. Справедливо следующее асимптотическое разложение:

$$
F(\mu)=\frac{1}{\mu} \int_{0}^{\infty} f(x) d x-\frac{2}{3} f(0)-\frac{1}{15} \mu f^{\prime}(0)+O\left(\mu^{2}\right) \quad \text { nри } \mu \rightarrow 0 .
$$

ДокАЗАТЕЛЬСтво. Положим

$$
R(x)=(2 x+1) f(\mu x(x+1))
$$

и заметим, что

$$
\int_{0}^{\infty} R(x) d x=\frac{1}{\mu} \int_{0}^{\infty} f(\mu x(x+1)) d(\mu x(x+1))=\frac{1}{\mu} \int_{0}^{\infty} f(x) d x .
$$

Вычислим производные $R$ до порядка 5 в $x=0$ :

$$
\begin{aligned}
R(0) & =f(0) \\
R^{\prime}(0) & =2 f(0)+\mu f^{\prime}(0) ; \\
R^{\prime \prime}(0) & =6 \mu f^{\prime}(0)+\mu^{2} f^{\prime \prime}(0) \\
R^{\prime \prime \prime}(0) & =12 \mu f^{\prime}(0)+12 \mu^{2} f^{\prime \prime}(0)+\mu^{3} f^{\prime \prime \prime}(0) ; \\
R^{(4)}(0) & =60 \mu^{2} f^{\prime \prime}(0)+20 \mu^{3} f^{\prime \prime \prime}(0)+\mu^{4} f^{(4)}(0) ; \\
R^{(5)}(0) & =120 \mu^{2} f^{\prime \prime}(0)+180 \mu^{3} f^{\prime \prime \prime}(0)+30 \mu^{4} f^{(4)}(0)+\mu^{5} f^{(5)}(0),
\end{aligned}
$$

И

$$
\begin{array}{r}
R^{(6)}(x)=840 \mu^{3}(2 x+1) f^{(3)}(\mu x(x+1))+420 \mu^{4}(2 x+1)^{3} f^{(4)}(\mu x(x+1)) \\
+42 \mu^{5}(2 x+1)^{5} f^{(5)}(\mu x(x+1))+\mu^{6}(2 x+1)^{7} f^{(6)}(\mu x(x+1)) .
\end{array}
$$


Далее мы используем формулу суммирования Эйлера-Маклорена (см., например, [18])

$$
\sum_{n=0}^{\infty} R(n)=\int_{0}^{\infty} R(x) d x+\frac{1}{2} R(0)-\sum_{i=2}^{k} \frac{B_{i}}{i !} R^{(i-1)}(0)-\int_{0}^{\infty} \frac{B_{k}(x)}{k !} R^{(k)}(x) d x,
$$

где $B_{k}$ - числа Бернулли: $B_{2}=1 / 6, B_{3}=0, B_{4}=-1 / 30, B_{5}=0, B_{6}=1 / 42, \ldots$, а $B_{k}(x)$ - периодические многочлены Бернулли. Используя (3.76) с $k=6$ и учитывая (3.73) и (3.74), получаем

$$
\begin{aligned}
F(\mu)=- & R(0)+\sum_{n=0}^{\infty} R(n)=\frac{1}{\mu} \int_{0}^{\infty} f(x) d x-\frac{1}{2} f(0)-\frac{1}{12}\left(2 f(0)+\mu f^{\prime}(0)\right) \\
& +\frac{1}{720}\left(12 \mu f^{\prime}(0)+12 \mu^{2} f^{\prime \prime}(0)+\mu^{3} f^{\prime \prime \prime}(0)\right) \\
& -\frac{1}{42 \cdot 720}\left(120 \mu^{2} f^{\prime \prime}(0)+180 \mu^{3} f^{\prime \prime \prime}(0)+30 \mu^{4} f^{(4)}(0)+\mu^{5} f^{(5)}(0)\right) \\
& -\int_{0}^{\infty} \frac{B_{6}(x)}{6 !} R^{(6)}(x) d x .
\end{aligned}
$$

Это дает (3.72), если остаточный интегральный член имеет порядок $O\left(\mu^{2}\right)$. Периодические многочлены Бернулли очевидно ограничены на $(0, \infty)$. Поэтому вклад каждого члена суммы (3.75) в интеграл будет порядка $\mu^{2}$. Например, последний член дает вклад

$$
\mu^{6} \int_{0}^{\infty} x^{7} g\left(\mu x^{2}\right) d x=\frac{1}{2} \mu^{2} \int_{0}^{\infty} y^{3} g(y) d y .
$$

Три оставшихся члена в (3.75) рассматриваются аналогично. Лемма доказана.

3.2.2.2. Неравенства на сферах: $\mathbb{S}^{3}$. На трехмерной сфере $\mathbb{S}^{3}$ мы рассмотрим только один пример с $l=1$ и $m=2$, так что $\theta=1 / 2$. Обозначим $A=(-\Delta)^{2}, B=-\Delta$, и пусть

$$
\mathbb{A}(\lambda)=(-\Delta)^{2}-\lambda \Delta .
$$

Функция Грина оператора $\mathbb{A}(\lambda)$ есть

$$
G_{\lambda}(x, \xi)=\sum_{n=1}^{\infty} \sum_{k=1}^{(n+1)^{2}} \frac{Y_{n}^{k}(\xi) Y_{n}^{k}(x)}{n(n+2)(n(n+2)+\lambda)},
$$

и, вновь используя $(3.57)$, видим, что $G_{\lambda}(\xi, \xi)$ не зависит от $\xi$ :

$$
G(\lambda)=G_{\lambda}(\xi, \xi)=\frac{1}{2 \pi^{2}} \sum_{n=1}^{\infty} \frac{(n+1)^{2}}{n(n+2)(n(n+2)+\lambda)} \quad \text { для любого } \xi \in \mathbb{S}^{3} .
$$

Нам сейчас не нужен аналог леммы 3.5, поскольку ряд (3.77) суммируется в явном виде [14]:

$$
G(\lambda)=\frac{1}{2 \pi^{2}}\left[\frac{\sqrt{\lambda-1}}{\lambda} \frac{\pi}{2} \operatorname{coth} \pi \sqrt{\lambda-1}-\frac{\lambda-1}{\lambda^{2}}+\frac{1}{4 \lambda}\right] .
$$


Применяя теорему 2.2 , где $\theta=1 / 2$, находим

$$
\sup _{\lambda \geqslant 0} \sqrt{\lambda} G(\lambda)=\lim _{\lambda \rightarrow \infty} \sqrt{\lambda} G(\lambda)=\frac{1}{4 \pi},
$$

что дает следующее точное неравенство на $\mathbb{S}^{3}[14]$ :

$$
\|u\|_{\infty}^{2} \leqslant \frac{1}{2 \pi}\|\nabla u\|\|\Delta u\|
$$

Для получения степенного разложения $G(\lambda)$ при $\lambda \rightarrow \infty$ мы просто заменим $\operatorname{coth} \pi \sqrt{\lambda-1}$ на 1 , что дает

$$
G(\lambda)=\frac{1}{2 \pi^{2}}\left[\frac{\pi}{2} \lambda^{-1 / 2}-\frac{3}{4} \lambda^{-1}-\frac{\pi}{4} \lambda^{-3 / 2}+O\left(\lambda^{-2}\right)\right],
$$

так что (2.41) выполняется с

$$
g_{1}=\frac{1}{4 \pi}, \quad g_{2}=-\frac{3}{8 \pi^{2}}, \quad g_{3}=-\frac{1}{8 \pi} .
$$

Используя предложение 2.1, получаем следующий результат.

Tеорема 3.6. Решение $\mathbb{V}(D)$ экстремалъной задачи

$$
\mathbb{V}(\xi, D):=\sup \left\{|u(\xi)|^{2}: u \in \bar{H}^{2}\left(\mathbb{S}^{3}\right),\|\nabla u\|^{2}=1,\|\Delta u\|^{2}=D\right\}
$$

не зависит от $\xi \in \mathbb{S}^{3}$ и допускает следующее асимптотическое разложение при $D \rightarrow \infty$ :

$$
\mathbb{V}(D)=\frac{1}{2 \pi} D^{1 / 2}-\frac{3}{4 \pi^{2}}-\frac{9+4 \pi^{2}}{16 \pi^{3}} D^{-1 / 2}+O\left(D^{-1}\right)
$$

Третий член в (3.83) отрицательный, поэтому

$$
\mathbb{V}(D)<\frac{1}{2 \pi} \sqrt{D}-\frac{3}{4 \pi^{2}}
$$

для всех $D \geqslant D_{0}$, где $D_{0}$ достаточно велико. Аналогично теореме 3.3 и лемме 3.3, используя явную формулу (3.78), получаем следующий результат.

Лемма 3.6. Неравенство (3.84) выполнено для всех $D \geqslant \sqrt{3}$.

ДокАЗАтельство. По теореме 2.3 для $D \geqslant \sqrt{3}$ (первое собственное значение $(-\Delta)^{1 / 2}$ на $\mathbb{S}^{3}$ есть $\left.\sqrt{3}\right)$ имеем

$$
\mathbb{V}(D)=\min _{\lambda \geqslant-\sqrt{3}}\{(\lambda+D) G(\lambda)\} \leqslant\left.\{(\lambda+D) G(\lambda)\}\right|_{\lambda=D}=2 D G(D) .
$$


Поэтому, используя (3.78) и неравенство $\sqrt{D-1} \leqslant \sqrt{D}-1 /(2 \sqrt{D})-1 /\left(8 D^{3 / 2}\right)$ и заменяя $\operatorname{coth} \pi \sqrt{D-1}$ в отрицательных членах на 1 , получаем

$$
\begin{aligned}
\mathbb{V}(D)-\frac{1}{2 \pi} \sqrt{D}+\frac{3}{4 \pi^{2}} & \leqslant 2 D G(D)-\frac{1}{2 \pi} \sqrt{D}+\frac{3}{4 \pi^{2}} \\
& =\frac{1}{2 \pi}\left(\sqrt{D-1} \operatorname{coth} \pi \sqrt{D-1}-\sqrt{D}+\frac{2}{\pi D}\right) \\
& \leqslant \frac{1}{2 \pi}\left(\sqrt{D}(\operatorname{coth} \pi \sqrt{D-1}-1)-\frac{1}{2 \sqrt{D}}-\frac{1}{8 D^{3 / 2}}+\frac{2}{\pi D}\right) \\
& =\frac{1}{2 \pi D^{3 / 2}}\left(D^{2}(\operatorname{coth} \pi \sqrt{D-1}-1)-\frac{D}{2}-\frac{1}{8}+\frac{2 \sqrt{D}}{\pi}\right) \\
& =\frac{1}{2 \pi D^{3 / 2}}\left(F_{1}(D)-F_{2}(D)\right)<0,
\end{aligned}
$$

так как функция $F_{1}(D)=D^{2}(\operatorname{coth} \pi \sqrt{D-1}-1)$ убывает при $D \geqslant \sqrt{3}$ и $F_{1}(D) \leqslant$ $F_{1}(\sqrt{3})=3 \operatorname{coth}(\pi \sqrt{\sqrt{3}-1}-1)=0.0278 \ldots$, а функция $F_{2}(D)=D / 2+1 / 8-$ $2 \sqrt{D} / \pi$ возрастает и $F_{2}(D) \geqslant F_{2}(\sqrt{3})=\sqrt{3} / 2+1 / 8-2 \sqrt[4]{3} / \pi=0.153 \ldots$. Лемма доказана.

В качестве следствия получаем уточнение неравенства (3.80).

Tеорема 3.7. Для $u \in \bar{H}^{2}\left(\mathbb{S}^{3}\right)$

$$
\|u\|_{\infty}^{2} \leqslant \frac{1}{2 \pi}\|\nabla u\|\|\Delta u\|-\frac{3}{4 \pi^{2}}\|\nabla u\|^{2},
$$

где обе постоянные точные, а экстремальной функции нет.

ЗАмЕчАниЕ 3.4. Коэффициент при главном члене в асимптотических разложениях (3.62), (3.83) совпадает с соответствующей постоянной в случае $\mathbb{R}^{n}$.

\section{3. Многообразия с границей.}

3.3.1. Неравенство первого порядка на полуоси. Мы рассмотрим несколько неравенств на полуоси $\mathbb{R}_{+}=(0, \infty)$. На всей прямой $\mathbb{R}$ справедливо неравенство

$$
\|u\|_{\infty}^{2} \leqslant\|u\|\left\|u^{\prime}\right\|
$$

где $u \in H^{1}(\mathbb{R})$, постоянная 1 точная, и существует единственная экстремальная функция $u_{*}(x)=e^{-|x|}$. Используя продолжение нулем, видим, что на $\mathbb{R}_{+}$ неравенство (3.87) по-прежнему выполняется, но экстремальной функции нет, поскольку $u_{*}(x)>0$. С помощью развитой выше теории можно получить это неравенство в уточненной форме

$$
u(\xi)^{2} \leqslant\|u\|\left\|u^{\prime}\right\|\left(1-e^{-2 \xi}\right), \quad u \in H_{0}^{1}\left(\mathbb{R}_{+}\right) .
$$

Действительно, функция Грина оператора

$$
\mathbb{A} u=-u^{\prime \prime}+u, \quad u(0)=u(\infty)=0,
$$


есть

$$
G(x, \xi)= \begin{cases}e^{-\xi} \sinh x, & x<\xi, \\ e^{-x} \sinh \xi, & x \geqslant \xi .\end{cases}
$$

Как и в лемме 2.1, отсюда получаем

$$
u(\xi)^{2} \leqslant\left(\left\|u^{\prime}\right\|^{2}+\|u\|^{2}\right) G(\xi, \xi)=\left(\left\|u^{\prime}\right\|^{2}+\|u\|^{2}\right) \frac{1-e^{-2 \xi}}{2},
$$

что эквивалентно неравенству (3.88).

3.3.2. Неравенство для оператора Бесселя на полуоси и на отрезке. Мы теперь рассмотрим одномерное неравенство на полуоси $\mathbb{R}_{+}$с $A u=-u^{\prime \prime}-u /\left(4 x^{2}\right)$ и $B u=u$. Хотя соответствующая точная постоянная была найдена в [10] в связи с неравенствами Либа-Тирринга для сферически симметричных потенциалов (см. также [9], [12]), мы включаем этот пример для иллюстрации нашего общего подхода. Во-первых, в силу одномерного неравенства Харди

$$
\int_{0}^{\infty} \frac{u(x)^{2}}{x^{2}} d x \leqslant 4 \int_{0}^{\infty}\left(u^{\prime}(x)\right)^{2} d x
$$

оператор $A$ неотрицательный. В соответствии с общей теорией из раздела 2 для нахождения точной постоянной $C=C(\xi)$ в неравенстве

$$
\|u\|_{\infty}^{2} \leqslant C_{\mathbb{R}_{+}}\left(\int_{0}^{\infty}\left(\left(u^{\prime}(x)\right)^{2}-\frac{u(x)^{2}}{4 x^{2}}\right) d x\right)^{1 / 2}\|u\|
$$

нам надо выписать функцию Грина оператора

$$
\mathbb{A}(\lambda) u=-u^{\prime \prime}-\frac{u}{4 x^{2}}+\lambda u
$$

с условиями Дирихле $u(0)=u(\infty)=0$ :

$$
G_{\lambda}(x, \xi)= \begin{cases}\sqrt{x \xi} K_{0}(\sqrt{\lambda} \xi) I_{0}(\sqrt{\lambda} x), & x<\xi \\ \sqrt{x \xi} K_{0}(\sqrt{\lambda} x) I_{0}(\sqrt{\lambda} \xi), & x>\xi\end{cases}
$$

где $K_{0}$ и $I_{0}-$ модифицированные функции Бесселя нулевого порядка. Действительно, функции $\sqrt{x} I_{0}(\sqrt{\lambda} x)$ и $\sqrt{x} K_{0}(\sqrt{\lambda} x)$ обе удовлетворяют однородному уравнению $\mathbb{A}(\lambda) u=0$, причем первая функция обращается в нуль в нуле, а вторая на бесконечности. Условие скачка $G_{x}^{\prime}(\xi+0, \xi)-G_{x}^{\prime}(\xi-0, \xi)=1$ выполнено в силу известного тождества для вронскиана и производных функций Бесселя:

$$
I_{0}(x) K_{0}^{\prime}(x)-I_{0}^{\prime}(x) K_{0}(x)=-\frac{1}{x}, \quad I_{0}^{\prime}(x)=I_{1}(x), \quad K_{0}^{\prime}(x)=-K_{1}(x) .
$$

Поэтому постоянная $C(\xi)$ в теореме 2.2 (обозначавшаяся там $K(\xi)$ ) в действительности не зависит от $\xi$ и выражается следующим образом:

$$
\begin{aligned}
C(\xi) & =2 \sup _{\lambda \geqslant 0} \sqrt{\lambda} G_{\lambda}(\xi, \xi)=2 \sup _{\lambda \geqslant 0} \sqrt{\lambda} \xi K_{0}(\sqrt{\lambda} \xi) I_{0}(\sqrt{\lambda} \xi) \\
& =2 \sup _{r \geqslant 0} r K_{0}(r) I_{0}(r)=\left.2 r K_{0}(r) I_{0}(r)\right|_{r=r_{*}=1.075 \ldots} \\
& =2 \cdot 0.533 \ldots=1.06 \ldots=: C_{\mathbb{R}_{+}} .
\end{aligned}
$$


Кроме того, для любого фиксированного $\xi>0$ неравенство (3.89) превращается в равенство при $u_{*}(x)=G_{\lambda_{*}}(x, \xi)$, где $\lambda_{*}=\lambda_{*}(\xi)=r_{*}^{2} / \xi^{2}$ и $\left\|u_{*}\right\|_{\infty}=u_{*}(\xi)$. Заметим также, что $I_{0}(x) \sim \sqrt{\frac{1}{2 \pi x}} e^{x}$ и $K_{0}(x) \sim \sqrt{\frac{\pi}{2 x}} e^{-x}$ при $x \rightarrow \infty$, следовательно,

$$
\lim _{r \rightarrow \infty} r K_{0}(r) I_{0}(r)=\frac{1}{2}
$$

Несколько сложнее технически рассмотрение неравенства на конечном интервале:

$$
\|u\|_{\infty}^{2} \leqslant C_{\text {int }}\left(\int_{0}^{1}\left(\left(u^{\prime}(x)\right)^{2}-\frac{u(x)^{2}}{4 d(x)^{2}}\right) d x\right)^{1 / 2}\left(\int_{0}^{1} u(x)^{2} d x\right)^{1 / 2},
$$

где $d(x)$ - расстояние от $x$ до концов отрезка $(0,1): d(x)=x, x \leqslant 1 / 2, d(x)=$ $1-x, 1 \geqslant x \geqslant 1 / 2$.

Надо выписать функцию Грина $G_{\lambda}(x, \xi)$ для задачи

$$
\mathbb{A}(\lambda) u=-u^{\prime \prime}-\frac{u}{4 d(x)^{2}}+\lambda u, \quad u(0)=u(1)=0 .
$$

Положим $\lambda=a^{2}$. При $x \in(0,1 / 2)$ общее решение однородного уравнения $\mathbb{A}(\lambda) u=0$ есть $c_{1} \sqrt{x} I_{0}(a x)+c_{2} \sqrt{x} K_{0}(a x)$, а при $x \in(1 / 2,1)$ общее решение есть $c_{1} \sqrt{1-x} I_{0}(a(1-x))+c_{2} \sqrt{1-x} K_{0}(a(1-x))$. Пусть для определенности $1 / 2<\xi<1$. Построим на всем отрезке $x \in(0,1)$ какое-нибудь $C^{1}$-гладкое решение $w_{1}(x)$ однородного уравнения $\mathbb{A}(\lambda) w_{1}=0$, выходящее из нуля при $x=0$. В силу вышесказанного оно имеет вид

$$
w_{1}(x)= \begin{cases}\sqrt{x} I_{0}(a x), & 0<x<1 / 2, \\ A \sqrt{1-x} I_{0}(a(1-x))+B \sqrt{1-x} K_{0}(a(1-x)), & 1 / 2<x<1\end{cases}
$$

где $A$ и $B$ - постоянные, которые определяются из условия $C^{1}$-гладкости функции $w_{1}(x)$ в точке $x=1 / 2$. Приравнивая в точке $x=1 / 2$ значение функций и производных справа и слева, получаем линейную систему для нахождения $A$ и $B$, решая которую, находим

$$
\begin{aligned}
& A=A(a)=1-I_{0}\left(\frac{a}{2}\right) K_{0}\left(\frac{a}{2}\right)-a I_{1}\left(\frac{a}{2}\right) K_{0}\left(\frac{a}{2}\right), \\
& B=B(a)=I_{0}\left(\frac{a}{2}\right)^{2}+a I_{0}\left(\frac{a}{2}\right) I_{1}\left(\frac{a}{2}\right) .
\end{aligned}
$$

Итак, для $w_{1}(x)$ при $x \in(0,1)$ имеем $\mathbb{A}(\lambda) w_{1}(x)=0, w_{1}(0)=0$.

Для $x>1 / 2$ решение однородного уравнения $\mathbb{A}(\lambda) w_{2}=0$ с $w_{2}(1)=0$ есть

$$
w_{2}(x)=\sqrt{1-x} I_{0}(a(1-x)) .
$$

Осталось вычислить вронскиан, который в силу теоремы Остроградского-Лиувилля не зависит от $x$ и который мы можем вычислить при $x>1 / 2$, где мы 
знаем оба решения. Пользуясь (3.91) (и учитывая, что вклад от члена с множителем $A$ равен нулю), находим $W=w_{1}(x) w_{2}^{\prime}(x)-w_{1}^{\prime}(x) w_{2}(x)=-B(a)$. Отсюда в силу известной общей формулы получаем явное выражение для $G_{\lambda}(x, \xi)$ :

$$
G_{\lambda}(x, \xi)=\frac{1}{B(a)} \begin{cases}w_{1}(x) w_{2}(\xi), & 1 / 2 \leqslant x \leqslant \xi, \\ w_{1}(\xi) w_{2}(x), & 1 / 2 \leqslant \xi \leqslant x,\end{cases}
$$

и явное выражение для ключевой функции из теоремы 2.2 :

$$
\begin{aligned}
\sqrt{\lambda} G_{\lambda}(\xi, \xi)= & a G_{a^{2}}(\xi, \xi)=: S(a, \xi) \\
= & \frac{1-I_{0}(a / 2) K_{0}(a / 2)-a I_{1}(a / 2) K_{0}(a / 2)}{I_{0}(a / 2)^{2}+a I_{0}(a / 2) I_{1}(a / 2)} a(1-\xi) I_{0}(a(1-\xi))^{2} \\
& \quad+a(1-\xi) I_{0}(a(1-\xi)) K_{0}(a(1-\xi))
\end{aligned}
$$

для $\xi \in[1 / 2,1]$ и симметричное выражение для $\xi \in[1,1 / 2]$ (с заменой всюду $1-\xi$ на $\xi)$.

Используя асимптотические разложения функций $I_{0}(x), I_{1}(x)$ и $K_{0}(x)$ при $x \rightarrow \infty$, можно показать, что равномерно по $\xi \in[0,1]$ первый член в $(3.96)$ имеет порядок $O\left(1 / a^{3}\right)$ при $a \rightarrow \infty$. Поэтому при больших $a \geqslant a_{0}$ поведение функции $\sqrt{\lambda} G_{\lambda}(\xi, \xi)$ определяется вторым слагаемым, а его поведение мы знаем (см. (3.92), (3.93)). Это объясняет появление двух "рожков" высоты, стремящейся к $0.533 \ldots$, в симметрично расположенных точках $\xi=r_{*} / a$ и $\xi=1-r_{*} / a$ (см. левый график на рис. 3 ).
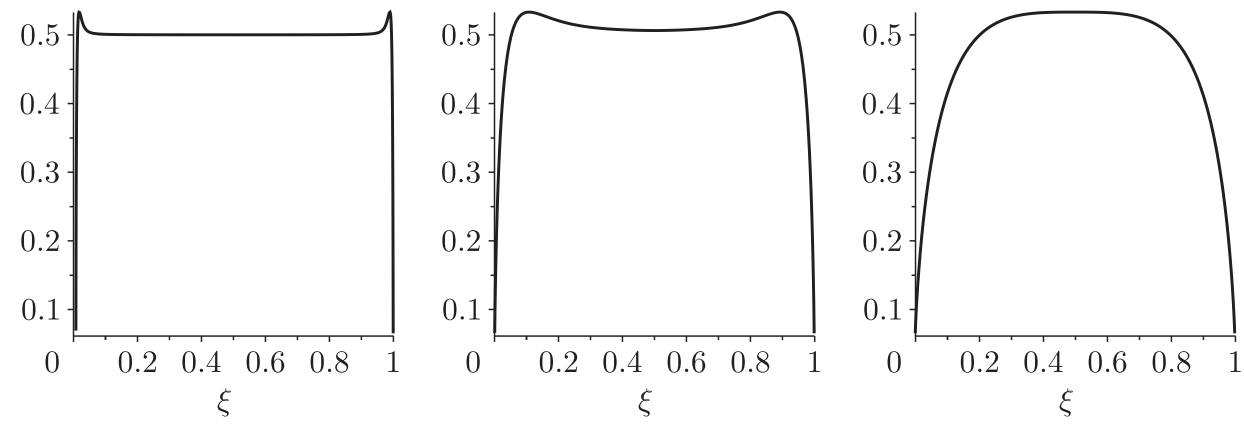

Рис. 3. График функции $\xi \mapsto \sqrt{\lambda} G_{\lambda}(\xi, \xi)=S(a, \xi)$ при $a=100$ ("рожки"), $a=10$ и $a=2.668 \ldots$ (глобальный максимум при $\xi=1 / 2$, равный $0.539285 \ldots)$

Следовательно, если при небольших $0<a<a_{0}$ в какой-то точке $\xi_{*}$ значение $S(a, \xi)$ больше, чем $0.533 \ldots$, то весь бесконечный промежуток $\left(a_{0}, \infty\right)$ можно не рассматривать. Так оно и есть. Вычисления на компьютере показывают, что при небольших $a$ функция $S(a, \xi)$ возрастает на $\xi \in[0,1 / 2]$, а максимум, равный $0.5392859275>0.533 \ldots$, достигается в $\xi=1 / 2$ при $a=a_{*}=2.668904$ (см. правый график на рис. 3).

Кроме того, для $\xi=1 / 2$ выражение (3.96) существенно упрощается:

$$
\sqrt{\lambda} G_{\lambda}\left(\frac{1}{2}, \frac{1}{2}\right)=S\left(a, \frac{1}{2}\right)=\frac{(a / 2) I_{0}(a / 2)}{I_{0}(a / 2)+a I_{1}(a / 2)} .
$$


Итак, мы нашли точную постоянную в неравенстве (3.94) и единственную экстремальную функцию:

$$
C_{\text {int }}=2 \cdot 0.539285=1.078571855, \quad u_{*}(x)=G_{a_{*}^{2}}\left(x, \frac{1}{2}\right) .
$$

3.3.3. Неравенство первого порядка на отрезке. В этом пункте мы рассмотрим корректирующий член для неравенства

$$
\|u\|_{\infty}^{2} \leqslant 1 \cdot\|u\|\left\|u^{\prime}\right\|, \quad u \in H_{0}^{1}(0, L),
$$

на отрезке $(0, L)$ с нулевыми граничными условиями. Постоянная 1 точная, поскольку она точная для неравенства (3.87) на всей оси $\mathbb{R}$, и мы можем использовать продолжение нулем. Единственная экстремальная функция в (3.87) не обращается в нуль, поэтому в (3.97) нет экстремальной функции. Можно было бы ожидать, что неравенство, подобное (3.22), верно и для случая конечного интервала:

$$
\|u\|_{\infty}^{2} \leqslant\|u\|\left\|u^{\prime}\right\|-c_{L}\|u\|^{2}, \quad u \in H_{0}^{1}(0, L) .
$$

В действительности, как мы сейчас покажем, $c_{L}=0$. Если (3.98) верно, то $c_{L}=c / L$, где $c$ - абсолютная постоянная. Далее, рассмотрим усеченную экстремальную функцию

$$
\varphi_{a}(x)= \begin{cases}e^{-x}, & 0 \leqslant x \leqslant a \\ e^{-a}(a+1-x), & a \leqslant x \leqslant a+1, \\ 0, & x \geqslant 0,\end{cases}
$$

и положим $\varphi_{a}(-x)=\varphi_{a}(x)$. Тогда

$$
\left\|\varphi_{a}\right\|_{\infty}=1, \quad\left\|\varphi_{a}\right\|^{2}=1-\frac{1}{3} e^{-2 a}, \quad\left\|\varphi_{a}^{\prime}\right\|^{2}=1+e^{-2 a} .
$$

Подставляя это в (3.98) и устремляя $a \mathrm{~K} \infty$, получаем, что (3.98) может выполняться лишь при $c=0$, как и утверждалось.

Однако корректор существует, но является экспоненциально малым. Точнее, справедлив следующий результат.

Tеорема 3.8. Пусть $u \in H_{0}^{1}(0, L)$. Тогда

$$
\|u\|_{\infty}^{2} \leqslant\|u\|\left\|u^{\prime}\right\|\left(1-2 e^{-L\left\|u^{\prime}\right\| /\|u\|}\right) .
$$

Коэффициенты двух слагаемых справа точные, а экстремальной функции нет.

ДокАЗАтЕЛЬСтво. Без ограничения общности можно считать, что $L=1$. Функция Грина краевой задачи

$$
-y^{\prime \prime}+\lambda y=\delta(x, \xi), \quad y(0)=y(1)=0
$$

есть

$$
G_{\lambda}(x, \xi)=\frac{1}{\lambda^{1 / 2} \sinh \lambda^{1 / 2}} \begin{cases}\sinh \lambda^{1 / 2} x \sinh \lambda^{1 / 2}(1-\xi), & 0 \leqslant x \leqslant \xi \\ \sinh \lambda^{1 / 2} \xi \sinh \lambda^{1 / 2}(1-x), & \xi \leqslant x \leqslant 1\end{cases}
$$


Максимум $G_{\lambda}(\xi, \xi)$ по $\xi$ достигается при $\xi=1 / 2$ :

$$
G_{\lambda}(\xi, \xi) \leqslant \frac{1}{2 \lambda^{1 / 2} \sinh \lambda^{1 / 2}}\left(\cosh \lambda^{1 / 2}-1\right)=\frac{1}{2 \lambda^{1 / 2}} \tanh \frac{\lambda^{1 / 2}}{2}=G_{\lambda}\left(\frac{1}{2}, \frac{1}{2}\right) .
$$

Как и в лемме 2.1, справедливо неравенство

$$
u(\xi)^{2} \leqslant G_{\lambda}(\xi, \xi)\left(\|u\|^{2}+\lambda\left\|u^{\prime}\right\|^{2}\right) \leqslant G_{\lambda}\left(\frac{1}{2}, \frac{1}{2}\right)\left(\|u\|^{2}+\lambda\left\|u^{\prime}\right\|^{2}\right),
$$

которое при $\xi=1 / 2$ обращается в равенство для $u(x)=\operatorname{const} G_{\lambda}(x, 1 / 2)$. Поэтому для

$$
\mathbb{V}(\xi, D):=\sup \left\{|u(\xi)|^{2}: u \in H_{0}^{1}(0,1),\|u\|^{2}=1,\left\|u^{\prime}\right\|^{2}=D\right\}
$$

выполняется неравенство $\mathbb{V}(\xi, D) \leqslant \mathbb{V}(1 / 2, D)$, и, как в $(2.30),(2.31)$, мы получаем параметрическое представление функции $\mathbb{V}(D):=\mathbb{V}(1 / 2, D)$ :

$$
D(\lambda)=\frac{h(\lambda)}{g(\lambda)}, \quad V(D(\lambda))=\frac{f(\lambda)^{2}}{g(\lambda)}, \quad \lambda \in\left[-\pi^{2}, \infty\right),
$$

где

$$
f(\lambda)=\frac{1}{2 \lambda^{1 / 2}} \tanh \frac{\lambda^{1 / 2}}{2}, \quad g(\lambda)=-f^{\prime}(\lambda), \quad h(\lambda)=f(\lambda)-\lambda g(\lambda) .
$$

Тогда неравенство (3.99) эквивалентно оценке

$$
\mathbb{V}(D) \leqslant \sqrt{D}\left(1-2 e^{-\sqrt{D}}\right) \text { для } D \geqslant \pi^{2} .
$$

Используя вариационное представление из теоремы 2.3, имеем

$$
\mathbb{V}(D)=\min _{\lambda \in\left(-\pi^{2}, \infty\right)} \frac{1}{2 \sqrt{\lambda}} \tanh \frac{\lambda^{1 / 2}}{2} \cdot(\lambda+D) .
$$

Для краткости положим $d:=\sqrt{D}$ и

$$
\lambda_{*}=\lambda_{*}(D):=D\left(1-2 \sqrt{D} e^{-\sqrt{D}}\right)^{2}=d^{2}\left(1-2 d e^{-d}\right)^{2} .
$$

Как мы увидим ниже, $\lambda_{*}(D)$ содержит первые два члена асимптотического разложения при $D \rightarrow \infty$ единственного решения $\lambda=\lambda(D)$ первого уравнения в (3.101), одновременно являющегося уравнением для единственной точки $\lambda=\lambda(D)$, в которой достигается глобальный минимум в (3.104). На данный же момент мы просто подставим $\lambda_{*}(D)$ в правую часть (3.104). Тогда (3.103) будет доказано, если мы покажем, что следующее неравенство справедливо для всех $d \geqslant \pi$ :

$$
\frac{1}{2} \frac{1}{d\left(1-2 d e^{-d}\right)} \tanh \frac{d\left(1-2 d e^{-d}\right)}{2} \cdot\left(d^{2}\left(1-2 d e^{-d}\right)^{2}+d^{2}\right) \leqslant d\left(1-2 e^{-d}\right),
$$

или

$$
\tanh \frac{d\left(1-2 d e^{-d}\right)}{2} \cdot\left(\left(1-2 d e^{-d}\right)^{2}+1\right) \leqslant 2\left(1-2 e^{-d}\right)\left(1-2 d e^{-d}\right) .
$$


Далее воспользуемся тем, что $\tanh (u / 2) \leqslant 1-2 e^{-u}+2 e^{-2 u}$, и заметим, что функция $1-2 x+2 x^{2}=2(x-1 / 2)^{2}+1 / 2$ монотонно убывает при $x \in[0,1 / 2]$, так что

$$
\begin{aligned}
\tanh \frac{d\left(1-2 d e^{-d}\right)}{2} & \leqslant 1-2 e^{-d\left(1-2 d e^{-d}\right)}+2 e^{-2 d\left(1-2 d e^{-d}\right)} \\
& \leqslant 1-2 e^{-d}\left(1+2 d^{2} e^{-d}\right)+2 e^{-2 d}\left(1+2 d^{2} e^{-d}\right)^{2}
\end{aligned}
$$

где использовано неравенство $e^{-d\left(1-2 d e^{-d}\right)} \geqslant e^{-d}\left(1+2 d^{2} e^{-d}\right)$, следующее из $e^{x}>1+x$.

Учитывая вышесказанное, достаточно доказать для $d \geqslant \pi$ неравенство

$$
\begin{aligned}
F(d):= & \left(1-2 e^{-d}\left(1+2 d^{2} e^{-d}\right)+2 e^{-2 d}\left(1+2 d^{2} e^{-d}\right)^{2}\right)\left(\left(1-2 d e^{-d}\right)^{2}+1\right) \\
& -2\left(1-2 e^{-d}\right)\left(1-2 d e^{-d}\right) \leqslant 0
\end{aligned}
$$

Упростим $F(d)$ :

$$
F(d)=4 e^{-2 d} F_{1}(d)
$$

где

$$
\begin{gathered}
F_{1}(d)=-d^{2}+2 d^{2} e^{-d}+4 d^{3} e^{-d}+1-2 d e^{-d}+2 d^{2} e^{-2 d} \\
-8 d^{3} e^{-2 d}+8 d^{4} e^{-3 d}-8 d^{5} e^{-3 d}+8 d^{6} e^{-4 d}
\end{gathered}
$$

Отбрасывая все отрицательные слагаемые, кроме $-d^{2}$, получаем $F_{1}(d)<-d^{2}+1+F_{2}(d), \quad F_{2}(d):=2 d^{2} e^{-d}+4 d^{3} e^{-d}+2 d^{2} e^{-2 d}+8 d^{4} e^{-3 d}+8 d^{6} e^{-4 d}$.

Каждый член в $F_{2}(d)$ монотонно убывает при $d \geqslant \pi$, поэтому

$$
F_{1}(d)<-\pi^{2}+1+F_{2}(\pi)=-2.530 \ldots<0,
$$

что и доказывает (3.103).

Для объяснения нашего выбора $\lambda_{*}(D)$ в (3.105) найдем асимптотическое разложение при $D \rightarrow \infty$ обратной функции $\lambda=\lambda(D)$, поскольку $\lambda_{*}(D)$ содержит первые два члена этого разложения. Справедливы разложения

$$
\begin{aligned}
f(\lambda) & =\frac{1}{2 \lambda^{1 / 2}} \tanh \frac{\lambda^{1 / 2}}{2}=\frac{1}{2 \lambda^{1 / 2}} \frac{1-e^{-\lambda^{1 / 2}}}{1+e^{-\lambda^{1 / 2}}} \\
& =\frac{1}{2} \lambda^{-1 / 2}\left(1-2 e^{-\lambda^{1 / 2}}+2 e^{-2 \lambda^{1 / 2}}+\cdots\right), \\
g(\lambda) & =\frac{1}{4} \lambda^{-3 / 2}\left(1+\left(-2-2 \lambda^{1 / 2}\right) e^{-\lambda^{1 / 2}}+\left(2+4 \lambda^{1 / 2}\right) e^{-2 \lambda^{1 / 2}}+\cdots\right), \\
h(\lambda) & =\frac{1}{4} \lambda^{-1 / 2}\left(1+\left(-2+2 \lambda^{1 / 2}\right) e^{-\lambda^{1 / 2}}+\left(2-4 \lambda^{1 / 2}\right) e^{-2 \lambda^{1 / 2}}+\cdots\right)
\end{aligned}
$$

и

$$
\begin{aligned}
D(\lambda) & =\frac{h(\lambda)}{g(\lambda)}=\frac{\lambda\left(1+\left(-2+2 \lambda^{1 / 2}\right) e^{-\lambda^{1 / 2}}+\left(2-4 \lambda^{1 / 2}\right) e^{-2 \lambda^{1 / 2}}+\cdots\right)}{1+\left(-2-2 \lambda^{1 / 2}\right) e^{-\lambda^{1 / 2}}+\left(2+4 \lambda^{1 / 2}\right) e^{-2 \lambda^{1 / 2}}+\cdots} \\
& =\lambda+4 \lambda^{3 / 2} e^{-\lambda^{1 / 2}}+8 \lambda^{2} e^{-2 \lambda^{1 / 2}}+\cdots
\end{aligned}
$$


Поэтому обратная функция $\lambda(D)$ имеет асимптотику

$$
\lambda(D)=D\left(1-4 \sqrt{D} e^{-\sqrt{D}}+\cdots\right)=d^{2}\left(1-2 d e^{-d}+\cdots\right)^{2},
$$

первые два члена которой дают (3.105).

Наконец, найдем асимптотику $\mathbb{V}(D)$ при $D \rightarrow \infty$ :

$$
\begin{aligned}
\mathbb{V}(D)= & \frac{1}{2 \sqrt{\lambda(D)}} \tanh \frac{\lambda(D)^{1 / 2}}{2} \cdot(\lambda(D)+D) \\
= & \frac{1}{2} \frac{1}{d\left(1-2 d e^{-d}+\cdots\right)} \tanh \frac{d\left(1-2 d e^{-d}+\cdots\right)}{2} \\
& \quad \times\left(d^{2}\left(1-2 d e^{-d}+\cdots\right)^{2}+d^{2}\right) \\
= & d \tanh \frac{d\left(1-2 d e^{-d}+\cdots\right)}{2} \cdot\left(1-2 d e^{-d}+\cdots\right)\left(1+2 d e^{-d}+\cdots\right) \\
= & d \tanh \frac{d\left(1-2 d e^{-d}+\cdots\right)}{2} \cdot(1+\cdots)=d\left(1-2 e^{-d}+\cdots\right), \quad d=\sqrt{D},
\end{aligned}
$$

что и доказывает точность коэффициентов в (3.99) и завершает доказательство теоремы 3.8 .

ЗАмЕчАниЕ 3.5. Рассуждая аналогично предложению 2.1, можно выписать три члена асимптотического разложения $\mathbb{V}(D)$ при $D \rightarrow \infty$ :

$$
\mathbb{V}(D)=D^{1 / 2}-2 D^{1 / 2} e^{-D^{-1 / 2}}-2 D^{1 / 2}(D-1) e^{-2 D^{-1 / 2}}+o\left(e^{-3 D^{-1 / 2}+\varepsilon}\right) .
$$

Третий член отрицательный, поэтому неравенство (3.103) выполняется для всех достаточно больших $D$. Однако, как мы показали, (3.103) верно для всех $D \geqslant \pi^{2}$.

3.3.4. О неравенстве второго порядка на полуоси и на отрезке. Теперь мы хотим применить нашу теорию к одномерному неравенству второго порядка в области $D$,

$$
\|u\|_{\infty}^{2} \leqslant K\|u\|^{3 / 2}\left\|u^{\prime \prime}\right\|^{1 / 2}, \quad u \in H_{0}^{1}(D) \cap H^{2}(D),
$$

в двух случаях: полуоси $D=\mathbb{R}_{+}$и конечного интервала $D=(0,1)$. В обоих случаях $A u=u^{\prime \prime \prime \prime}, B u=u, \theta=3 / 4$ и $\|u\|_{A}=\left\|u^{\prime \prime}\right\|$.

Начнем с более простого случая полуоси. Тогда надо найти фундаментальное решение уравнения

$$
\mathbb{A}(\lambda) u(x)=u^{\prime \prime \prime \prime}(x)+\lambda u(x)=\delta(x-\xi), \quad x, \xi>0, \quad u(0)=u^{\prime \prime}(0)=0 .
$$

Оно находится методом отражения:

$$
G_{\lambda}(x, \xi)=\frac{\sqrt{2}}{4} \frac{1}{\lambda^{3 / 4}}\left[f_{0}\left(\frac{\lambda^{1 / 4}(x-\xi)}{\sqrt{2}}\right)-f_{0}\left(\frac{\lambda^{1 / 4}(x+\xi)}{\sqrt{2}}\right)\right],
$$

где

$$
\int_{-\infty}^{\infty} \frac{e^{-i x y} d x}{x^{4}+1}=\frac{\pi \sqrt{2}}{2} f_{0}\left(\frac{|y|}{\sqrt{2}}\right), \quad f_{0}(x)=e^{-|x|}(\cos x+\sin |x|),
$$


и $\frac{\sqrt{2}}{4} \frac{1}{\lambda^{3 / 4}} f_{0}\left(\frac{\lambda^{1 / 4}(x-\xi)}{\sqrt{2}}\right)$ - фундаментальное решение уравнения $\mathbb{A}(\lambda) u(x)=$ $\delta(x-\xi)$ на всей оси.

Поэтому для любого фиксированного $\xi>0$, следуя теореме 2.2 , находим

$\sup _{\lambda>0} \lambda^{3 / 4} G_{\lambda}(\xi, \xi)=\frac{\sqrt{2}}{4} \sup _{\lambda>0}\left(1-f_{0}\left(\sqrt{2} \lambda^{1 / 4} \xi\right)\right)=\frac{\sqrt{2}}{4} \sup _{t>0}\left(1-f_{0}(t)\right)=\frac{\sqrt{2}}{4}\left(1+e^{-\pi}\right)$,

так как $f_{0}(x)$ имеет положительные локальные максимумы в $x=2 \pi n$ (в 0 глобальный) и отрицательные локальные минимумы в $x=\pi+2 \pi n$ (в $\pm \pi$ глобальные). По теореме 2.2 получаем значение точной постоянной $K_{\mathbb{R}_{+}}=$ $(4 / 27)^{1 / 4}\left(1+e^{-\pi}\right)$ и семейство экстремальных функций

$$
u_{*}(x)=G_{\lambda(\xi)}(x, \xi)=f_{0}\left(\frac{\pi}{2}\left(\frac{x}{\xi}-1\right)\right)-f_{0}\left(\frac{\pi}{2}\left(\frac{x}{\xi}+1\right)\right),
$$

где $\xi>0$ произвольно и фиксировано, а $\lambda(\xi)=\pi^{4} /\left(4 \xi^{4}\right)$.

Переходим к случаю конечного интервала. Для того чтобы найти ключевую функцию Грина $G_{\lambda}(\xi, \xi)$, мы должны найти решение уравнения

$$
u^{\prime \prime \prime \prime}(x)+\lambda u(x)=\delta(x-\xi), \quad x, \xi \in(0,1), \quad u(0)=u^{\prime \prime}(0)=u(1)=u^{\prime \prime}(1)=0 .
$$

Используя ортонормированную систему собственных функций $\{\sqrt{2} \sin \pi n x\}_{n=1}^{\infty}$, получаем

$$
G_{\lambda}(x, \xi)=2 \sum_{n=1}^{\infty} \frac{\sin \pi n x \sin \pi n \xi}{\pi^{4} n^{4}+\lambda}
$$

и (полагая $\lambda=4 a^{4} \pi^{4}$ для упрощения дальнейших формул)

$$
G_{\lambda}(\xi, \xi)=\frac{2}{\pi^{4}} \sum_{n=1}^{\infty} \frac{\sin ^{2} \pi n \xi}{n^{4}+4 a^{4}}
$$

Затем, используя равенство $\sin ^{2} \pi n \xi=1 / 2-\left(e^{2 \pi i n \xi}+e^{-2 \pi i n \xi}\right) / 4$ и формулу суммирования Пуассона

$$
\sum_{n=-\infty}^{\infty} g(n+y)=\sqrt{2 \pi} \sum_{n=-\infty}^{\infty} e^{2 \pi i n y} \widehat{g}(2 \pi n)
$$

находим

$$
\begin{aligned}
\sum_{n=1}^{\infty} \frac{\sin ^{2} \pi n \xi}{n^{4}+4 a^{4}} & =\frac{1}{4}\left(\sum_{n=-\infty}^{\infty} \frac{1}{n^{4}+4 a^{4}}-\sum_{n=-\infty}^{\infty} \frac{e^{2 \pi i n \xi}}{n^{4}+4 a^{4}}\right) \\
& =\frac{1}{4} \frac{1}{(\sqrt{2} a)^{3}}\left(\sum_{n=-\infty}^{\infty} f(\sqrt{2} a \cdot 2 \pi n)-\sum_{n=-\infty}^{\infty} f(\sqrt{2} a \cdot 2 \pi(n+\xi))\right) \\
& =\frac{1}{4} \frac{1}{(\sqrt{2} a)^{3}} \frac{\pi \sqrt{2}}{2}\left(\sum_{n=-\infty}^{\infty} f_{0}(a \cdot 2 \pi n)-\sum_{n=-\infty}^{\infty} f_{0}(a \cdot 2 \pi(n+\xi))\right),
\end{aligned}
$$


где $f(y):=\frac{\pi \sqrt{2}}{2} f_{0}\left(\frac{|y|}{\sqrt{2}}\right), f_{0}(x)=e^{-|x|}(\cos x+\sin |x|)$, см. (3.111). Собирая все вместе, для ключевой функции в теореме 2.2 получаем

$$
\lambda^{3 / 4} G_{\lambda}(\xi, \xi)=\frac{\sqrt{2}}{4}\left(\sum_{n=-\infty}^{\infty} f_{0}(a \cdot 2 \pi n)-\sum_{n=-\infty}^{\infty} f_{0}(a \cdot 2 \pi(n+\xi))\right)=: \frac{\sqrt{2}}{4} S(a, \xi),
$$

где $\lambda=4 a^{4} \pi^{4}$. Для больших $a$ и $\xi \leqslant 1 / 2$ главные члены суть

$$
S(a, \xi)=f_{0}(0)-f_{0}(2 \pi a \xi)+O\left(e^{-2 \pi a}\right),
$$

и, поскольку $f_{0}(x)$ имеет отрицательный глобальный минимум в $\pi$, мы видим "рожок" высоты $e^{-\pi}$ над $f_{0}(0)=1$ при $\xi=1 /(2 a)$, дающий максимум $S(a, \xi)$ при больших $a$; см. рис. 4.
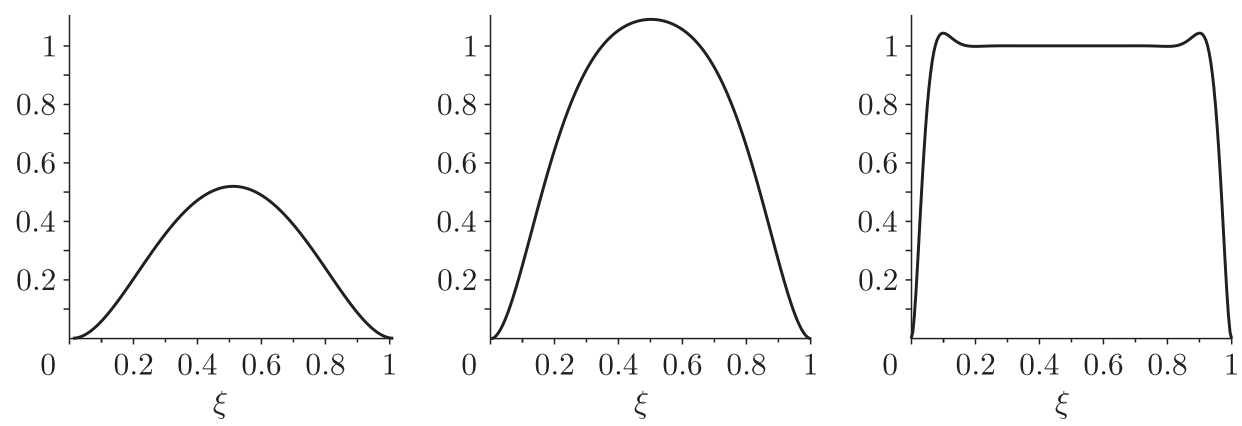

Рис. 4. Графики функции $\xi \mapsto S(a, \xi)$ при $a=0.5, a=1$ (глобальный максимум при $\xi=1 / 2$, равный $\operatorname{coth}(\pi / 2))$ и $a=10$ ("рожки")

Что же касается глобального максимума $S(a, \xi)$, мы видим, что если $a=1$ и $\xi=1 / 2$, то в первой и второй сумме в (3.114) посчитаны один за другим все максимумы и все отрицательные минимумы функции $f_{0}$ соответственно. Поэтому

$$
S(a, \xi) \leqslant S\left(1, \frac{1}{2}\right)=\operatorname{coth} \frac{\pi}{2} .
$$

Итак, мы доказали следующий результат.

Теорема 3.9. Точные постоянные в неравенстве (3.110) для полуоси и отрезка суть соответственно

$$
\begin{aligned}
K_{\mathbb{R}_{+}} & =\frac{\sqrt{2}}{\sqrt[4]{27}}\left(1+e^{-\pi}\right)=\frac{\sqrt{2}}{\sqrt[4]{27}} \cdot 1.0432 \ldots, \\
K_{\mathrm{int}} & =\frac{\sqrt{2}}{\sqrt[4]{27}} \operatorname{coth} \frac{\pi}{2}=\frac{\sqrt{2}}{\sqrt[4]{27}} \cdot 1.09033 \ldots
\end{aligned}
$$

Для полуоси семейство экстремальных функиий приведено в (3.112), а для интервала экстремальная функиия дается формулой (3.113), где $\lambda=4 \pi^{4}$ $u \xi=1 / 2$. 
ЗАмечАниЕ 3.6. Точная постоянная в неравенстве (3.110) для $u \in H_{0}^{2}(D)$ такая же, как в случае всей оси, а именно $\sqrt{2} / \sqrt[4]{27}$, поскольку можно использовать продолжение нулем.

3.3.5. Неравенство второго порядка в 3D. В заключение мы рассмотрим неравенство второго порядка в трехмерной области $\Omega \subseteq \mathbb{R}^{3}$, для которого переход от пространства $H_{0}^{2}(\Omega)$ к более широкому пространству $H^{2}(\Omega) \cap H_{0}^{1}(\Omega)$ не приводит к увеличению постоянной в соответствующем интерполяционном неравенстве. Это неравенство было получено в [28] некоторым специальным методом, тем не менее мы приводим его доказательство в рамках нашей общей теоремы 2.2 .

Теорема 3.10. Пусть $\Omega \subseteq \mathbb{R}^{3}$ - произвольная область. Обозначим через $\dot{H}_{0}^{1}(\Omega)$ замыкание $C_{0}^{\infty}(\Omega)$ в норме $\|\nabla \cdot\|_{L_{2}(\Omega)}$. Тогда для $u \in \dot{H}_{0}^{1}(\Omega) \cap\{u: \Delta u \in$ $\left.L_{2}(\Omega)\right\}$ справедливо неравенство

$$
\|u\|_{\infty}^{2} \leqslant \frac{1}{2 \pi}\|\nabla u\|\|\Delta u\|
$$

где постоянная точная, и экстремальной функиии нет, если $\Omega \neq \mathbb{R}^{3}$. В противном случае

$$
u_{*}(x)=\mathscr{F}^{-1}\left(\frac{1}{|\xi|^{2}+|\xi|^{4}}\right) .
$$

ДокАЗАТЕЛЬство. Сначала предположим, что $\Omega$ - ограниченная область с гладкой границей. Тогда из эллиптической регулярности следует, что

$$
u \in \dot{H}_{0}^{1}(\Omega) \cap\left\{u: \Delta u \in L_{2}(\Omega)\right\}=H^{2}(\Omega) \cap H_{0}^{1}(\Omega) .
$$

Согласно теореме 2.2 нам надо рассмотреть функцию Грина $G_{\lambda}(x, \xi)$ следующего эллиптического оператора четвертого порядка:

$$
\begin{gathered}
\left(\Delta^{2}-\lambda \Delta\right) G_{\lambda}(x, \xi)=-\Delta(-\Delta+\lambda) G_{\lambda}(x, \xi)=\delta(x-\xi), \\
G_{\lambda}(x, \xi)=\Delta G_{\lambda}(x, \xi)=0, \quad \xi \in \Omega, \quad x \in \partial \Omega .
\end{gathered}
$$

Обозначим через $g_{\mathrm{D}}(x, \xi)$ функцию Грина лапласиана Дирихле:

$$
-\Delta g_{\mathrm{D}}(x, \xi)=\delta(x-\xi), \quad g_{\mathrm{D}}(x, \xi)=0, \quad \xi \in \Omega, \quad x \in \partial \Omega
$$

и через $g_{\mathrm{H}}(x, \xi)$ функцию Грина для уравнения Гельмгольца:

$$
(-\Delta+\lambda) g_{\mathrm{H}}(x, \xi)=\delta(x-\xi), \quad g_{\mathrm{H}}(x, \xi)=0, \quad \xi \in \Omega, \quad x \in \partial \Omega .
$$

По принципу максимума

$$
0<g_{\mathrm{D}}(x, \xi)<\frac{1}{4 \pi|x-\xi|}, \quad 0<g_{\mathrm{H}}(x, \xi)<\frac{e^{-\sqrt{\lambda}|x-\xi|}}{4 \pi|x-\xi|},
$$

где функции справа - это соответствующие фундаментальные решения в $\mathbb{R}^{3}$. 
Поэтому

$$
\begin{aligned}
0 & <G_{\lambda}(x, \xi)=\int_{\Omega} g_{\mathrm{H}}(x, y) g_{\mathrm{D}}(y, \xi) d y<\int_{\Omega} \frac{e^{-\sqrt{\lambda}|x-y|}}{4 \pi|x-y|} \frac{1}{4 \pi|y-\xi|} d y \\
& <\int_{\mathbb{R}^{3}} \frac{e^{-\sqrt{\lambda}|x-y|}}{4 \pi|x-y|} \frac{1}{4 \pi|y-\xi|} d y=\frac{1}{(2 \pi)^{3}} \int_{\mathbb{R}^{3}} \frac{e^{i(\xi-x) z} d z}{|z|^{2}\left(|z|^{2}+\lambda\right)},
\end{aligned}
$$

где справа стоит фундаментальное решение уравнения (3.116) в $\mathbb{R}^{3}$. Следовательно,

$$
G_{\lambda}(\xi, \xi)<\frac{1}{(2 \pi)^{3}} \int_{\mathbb{R}^{3}} \frac{d z}{|z|^{2}\left(|z|^{2}+\lambda\right)}=\frac{1}{4 \pi \sqrt{\lambda}} .
$$

Теперь (3.115) следует из теоремы 2.2. Постоянная $1 /(2 \pi)$ очевидно точная, поскольку она точна для неравенства для $u \in H_{0}^{2}\left(\mathbb{R}^{3}\right)$ (см. (3.2)) и (в этом случае используем продолжение нулем) для $u \in H_{0}^{2}(\Omega) \subset \dot{H}_{0}^{1}(\Omega) \cap\{u: \Delta u \in$ $\left.L_{2}(\Omega)\right\}$.

Исчерпывая произвольную область $\Omega \subset \mathbb{R}^{3}$ ограниченными областями $\Omega_{n}$, можно показать, что (3.115) верно и в общем случае (см. детали в [28]). Теорема доказана.

ЗАмЕчАниЕ 3.7. В отличие от предыдущего примера, фундаментальное решение (3.116) в $\mathbb{R}^{3}$ положительно.

Авторы выражают благодарность А. А. Лаптеву и С. И. Похожаеву за многочисленные полезные обсуждения.

\section{Список литературы}

[1] В. В. Арестов, "Приближение неограниченных операторов ограниченными и родственные экстремальные задачи", УМН, 51:6(312) (1996), 89-124; англ. пер.: V.V. Arestov, "Approximation of unbounded operators by bounded operators and related extremal problems", Russian Math. Surveys, 51:6 (1996), 1093-1126.

[2] M. Bartuccelli, J. Deane, S. Zelik, "Asymptotic expansions and extremals for the critical Sobolev and Gagliardo-Nirenberg inequalities on a torus", Proc. Roy. Soc. Edinburgh Sect. A, 143:3 (2013), 445-482.

[3] О.В.Бесов, В. П. Ильин, С. М. Никольский, Интегральные представления функиий и теоремы вложения, Наука, М., 1975, 480 с.; англ. пер.: О. V. Besov, V.P. Il'in, S. M. Nikol'skii, Integral representations of functions and imbedding theorems, v. I, II, Scripta Series in Mathematics, ed. M. H. Taibleson, V. H. Winston \& Sons, Washington, DC; John Wiley \& Sons, New York-Toronto-London, 1978, 1979, viii+345 pp., viii+311 pp.

[4] H. Brezis, T. Gallouet, "Nonlinear Schrödinger evolution equations", Nonlinear Anal., 4:4 (1980), 677-681.

[5] F. Carlson, "Une inégalité", Ark. Mat. Astr. Fys., 25B:1 (1934), 1-5.

[6] J. Dolbeault, A. Laptev, M. Loss, "Lieb-Thirring inequalities with improved constants", J. Eur. Math. Soc. (JEMS), 10:4 (2008), 1121-1126.

[7] A. Eden, C. Foias, "A simple proof of the generalized Lieb-Thirring inequalities in one-space dimension", J. Math. Anal. Appl., 162:1 (1991), 250-254. 
[8] H. M. Edwards, Riemann's zeta function, reprint of the 1974 original, Dover Publications, Inc., Mineola, NY, 2001, xiv+315 pp.

[9] T. Ekholm, R. Frank, "Lieb-Thirring inequalities on the half-line with critical exponent", J. Eur. Math. Soc. (JEMS), 10:3 (2008), 739-755.

[10] R. Frank, A. Laptev, A Lieb-Thirring inequality for spherically symmetric potentials, preprint, 2007.

[11] В. Н. Габушин, "О наилучшем приближении оператора дифференцирования на полупрямой", Матем. заметки, 6:5 (1969), 573-582; англ. пер.: V. N. Gabushin, "On the best approximation of the differentiation operator on the half-line", Math. Notes, 6:5 (1969), 804-810.

[12] L. Geisinger, A. Laptev, T. Weidl, "Geometrical versions of improved Berezin-Li-Yau inequalities", J. Spectr. Theory, 1:1 (2011), 87-109.

[13] G. H. Hardy, "A note on two inequalities", J. London Math. Soc., s1-11:3 (1936), $167-170$.

[14] A. A. Ilyin, "Best constants in multiplicative inequalities for sup-norms", J. London Math. Soc. (2), 58:1 (1998), 84-96.

[15] A. A. Ilyin, "Best constants in Sobolev inequalities on the sphere and in Euclidean space", J. London Math. Soc. (2), 59:1 (1999), 263-286.

[16] A. A. Ilyin, "Lieb-Thirring inequalities on some manifolds", J. Spectr. Theory, 2:1 (2012), 57-78.

[17] Г.А. Калябин, "Точные константы в неравенствах для промежуточных производных (случай Габушина)", Функи. анализ и его прил., 38:3 (2004), 29-38; англ. пер.: G.A. Kalyabin, "Sharp constants in inequalities for intermediate derivatives (the Gabushin case)", Funct. Anal. Appl., 38:3 (2004), 184-191.

[18] В. И. Крылов, Приближенное вычисление интегралов, Гос. изд-во физ.-мат. лит., M., 1959, 327 с.; англ. пер.: V.I. Krylov, Approximate calculation of integrals, The Macmillan Co., New York-London, 1962, x+357 pp.

[19] L. Larsson, L. Maligranda, J. Pečarić, L.-E. Persson, Multiplicative inequalities of Carlson type and interpolation, World Scientific Publishing Co., Hackensack, NJ, 2006, xiv $+201 \mathrm{pp}$.

[20] E. H. Lieb, W.E. Thirring, "Inequalities for the moments of the eigenvalues of the Schrödinger Hamiltonian and their relation to Sobolev inequalities", Studies in mathematical physics: essays in honor of Valentine Bargmann, Princeton Ser. Phys., Princeton Univ. Press, Princeton, NJ, 1976, 269-303.

[21] Г. Г. Магарил-Ильяев, В. М. Тихомиров, "О неравенствах для производных колмогоровского типа", Матем. сб., 188:12 (1997), 73-106; англ. пер.: G. G. Маgaril-Il'yaev, V.M. Tikhomirov, "Kolmogorov-type inequalities for derivatives", $S b$. Math., 188:12 (1997), 1799-1832.

[22] И. М. Стейн, Г. Вейс, Введение в гармонический анализ на евклидовых пространствах, Мир, М., 1974, 336 с.; пер. с англ.: E. M. Stein, G. Weiss, Introduction to Fourier analysis on Euclidean spaces, Princeton Math. Ser., 32, Princeton Univ. Press, Princeton, NJ, 1971, x+297 pp.

[23] B. von Sz.-Nagy, "Über integralungleichungen zwischen einer Funktion und ihrer Ableitung", Acta Univ. Szeged. Sect. Sci. Math., 10 (1941), 64-74.

[24] Л.В. Тайков, "Неравенства типа Колмогорова и наилучшие формулы численного дифференцирования", Матем. заметки, 4:2 (1968), 233-238; англ. пер.: L. V. Taikov, "Kolmogorov-type inequalities and the best formulas for numerical differentiation", Math. Notes, 4:2 (1968), 631-634. 
[25] Е.К. Титчмарш, Теория функиий, ГИТТЛ, М., 1951, 506 с.; пер. с англ.: E. C. Titchmarsh, The theory of functions, 2nd ed., Oxford Univ. Press, London, 1939, x+454 pp.

[26] Х. Трибель, Теория интерполяции, функииональные пространства, дифференииальные операторы, Мир, М., 1980, 660 с.; пер. с англ.: Н. Triebel, Interpolation theory, function spaces, differential operators, VEB Deutscher Verlag der Wissenschaften, Berlin, 1978, 528 pp.

[27] Дж. Н. Ватсон, Теория бесселевых функиий, ИЛ, М., 1949, 798 с.; пер. с англ.: G. N. Watson, A treatise on the theory of Bessel functions, 2nd ed., Cambridge Univ. Press, Cambridge, England; The Macmillan Co., New York, 1944, vi+804 pp.

[28] Wenzheng Xie, "Integral representations and $L^{\infty}$ bounds for solutions of the Helmholtz equation on arbitrary open sets in $\mathbb{R}^{2}$ and $\mathbb{R}^{3}$ ", Differential Integral Equations, 8:3 (1995), 689-698.

\section{Сергей Витальевич Зелик}

(Sergey V. Zelik)

Поступила в редакцию

University of Surrey, Guildford, UK

27.10 .2013

E-mail: s.zelik@surrey.ac.uk

\section{Алексей Андреевич Ильин}

\section{(Aleksei A. Ilyin)}

Институт прикладной математики

им. М. В. Келдыша РАН;

Институт проблем передачи информации

им. А. А. Харкевича РАН

E-mail: ilyin@keldysh.ru 